Advances in Experimental Medicine and Biology 1137

Francisco Couto

Data and Text

Processing for

Health and Life

Sciences

OPEN

Springer 


\section{Advances in Experimental Medicine and Biology}

\section{Volume 1137}

\section{Editorial Board}

IRUN R. COHEN, The Weizmann Institute of Science, Rehovot, Israel ABEL LAJTHA, N.S. Kline Institute for Psychiatric Research, Orangeburg, NY, USA

JOHN D. LAMBRIS, University of Pennsylvania, Philadelphia, PA, USA RODOLFO PAOLETTI, University of Milan, Milano, Italy NIMA REZAEI, Tehran University of Medical Sciences, Children's Medical Center Hospital, Tehran, Iran 
More information about this series at http://www.springer.com/series/5584 
Francisco M. Couto

\section{Data and Text Processing for Health and Life Sciences}


Francisco M. Couto

LASIGE, Department of Informatics

Faculdade de Ciências, Universidade de Lisboa

Lisbon, Portugal

ISSN 0065-2598

ISSN 2214-8019 (electronic)

Advances in Experimental Medicine and Biology

ISBN 978-3-030-13844-8 $\quad$ ISBN 978-3-030-13845-5 (eBook)
https://doi.org/10.1007/978-3-030-13845-5

(C) The Editor(s) (if applicable) and The Author(s) 2019. This book is an open access publication. Open Access This book is licensed under the terms of the Creative Commons Attribution 4.0 International License (http://creativecommons.org/licenses/by/4.0/), which permits use, sharing, adaptation, distribution and reproduction in any medium or format, as long as you give appropriate credit to the original author(s) and the source, provide a link to the Creative Commons licence and indicate if changes were made.

The images or other third party material in this book are included in the book's Creative Commons licence, unless indicated otherwise in a credit line to the material. If material is not included in the book's Creative Commons licence and your intended use is not permitted by statutory regulation or exceeds the permitted use, you will need to obtain permission directly from the copyright holder.

The use of general descriptive names, registered names, trademarks, service marks, etc. in this publication does not imply, even in the absence of a specific statement, that such names are exempt from the relevant protective laws and regulations and therefore free for general use.

The publisher, the authors, and the editors are safe to assume that the advice and information in this book are believed to be true and accurate at the date of publication. Neither the publisher nor the authors or the editors give a warranty, express or implied, with respect to the material contained herein or for any errors or omissions that may have been made. The publisher remains neutral with regard to jurisdictional claims in published maps and institutional affiliations.

This Springer imprint is published by the registered company Springer Nature Switzerland AG. The registered company address is: Gewerbestrasse 11, 6330 Cham, Switzerland 
Aos meus pais, Francisco de Oliveira Couto e Maria Fernanda dos Santos Moreira Couto. 


\section{Preface}

During the last decades, I witnessed the growing importance of computer science skills for career advancement in Health and Life Sciences. However, not everyone has the skill, inclination, or time to learn computer programming. The learning process is usually time-consuming and requires constant practice, since software frameworks and programming languages change substantially overtime. This is the main motivation for writing this book about using shell scripting to address common biomedical data and text processing tasks. Shell scripting has the advantages of being: (i) nowadays available in almost all personal computers; (ii) almost immutable for more than four decades; (iii) relatively easy to learn as a sequence of independent commands; (iv) an incremental and direct way to solve many of the data problems that Health and Life professionals face.

During the last decades, I had the pleasure to teach introductory computer science classes to Life and Health and Life Sciences undergraduates. I used programming languages, such as Perl and Python, to address data and text processing tasks, but I always felt to lose a substantial amount of the time teaching the technicalities of these languages, which will probably change over time and are uninteresting for the majority of the students who do not intend to pursue advanced bioinformatics courses. Thus, the purpose of this book is to motivate and help specialists to automate common data and text processing tasks after a short learning period. If they become interested (and I hope some do), the book presents pointers to where they can acquire more advanced computer science skills.

This book does not intend to be a comprehensive compendium of shell scripting commands but instead an introductory guide for Health and Life specialists. This book introduces the commands as they are required to automate data and text processing tasks. The selected tasks have a strong focus on text mining and biomedical ontologies given my research experience and their growing relevance for Health and Life studies. Nevertheless, the same type of solutions presented in the book are also applicable to many other research fields and data sources. 


\section{Acknowledgments}

I am grateful to all the people who helped and encouraged me along this journey, especially to Rita Ferreira for all the insightful discussions about shell scripting.

I am also grateful for all the suggestions and corrections given by my colleague Prof. José Baptista Coelho and by my college students: Alice Veiros, Ana Ferreira, Carlota Silva, Catarina Raimundo, Daniela Matias, Inês Justo, João Andrade, João Leitão, João Pedro Pais, Konil Solanki, Mariana Custódio, Marta Cunha, Manuel Fialho, Miguel Silva, Rafaela Marques, Raquel Chora and Sofia Morais.

This work was supported by FCT through funding of DeST: Deep Semantic Tagger project, ref. PTDC/CCI-BIO/28685/2017 (http://dest.rd.ciencias. ulisboa.pt/), and LASIGE Research Unit, ref. UID/CEC/00408/2019. 


\section{Contents}

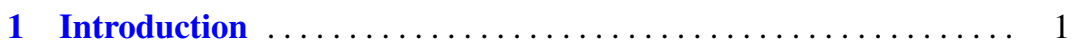

Biomedical Data Repositories.................... 1

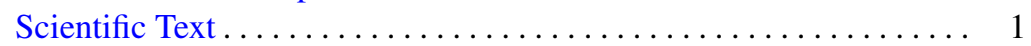

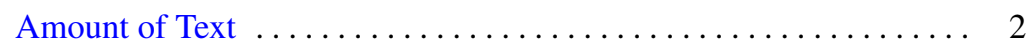

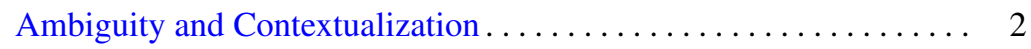

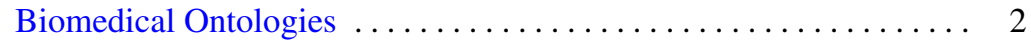

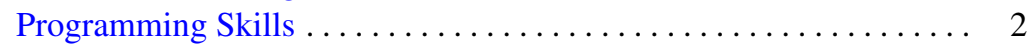

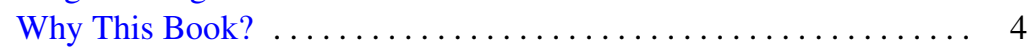

Third-Party Solutions $\ldots \ldots \ldots \ldots \ldots \ldots \ldots \ldots \ldots \ldots$

Simple Pipelines .................... 5

How This Book Helps Health and Life Specialists? ........... 5

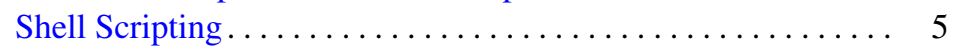

Text Files $\ldots \ldots \ldots \ldots \ldots \ldots \ldots \ldots \ldots \ldots \ldots \ldots \ldots \ldots . \ldots \ldots$

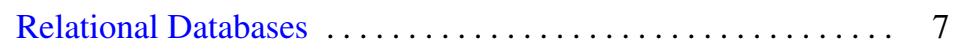

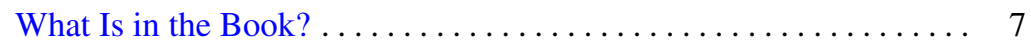

Command Line Tools ....................... 7

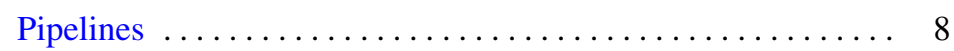

Regular Expressions $\ldots \ldots \ldots \ldots \ldots \ldots \ldots \ldots \ldots \ldots \ldots$

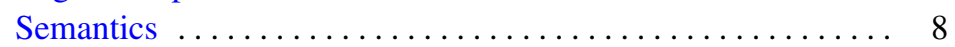

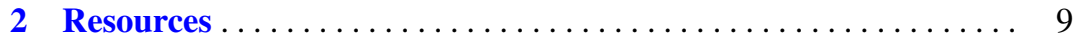

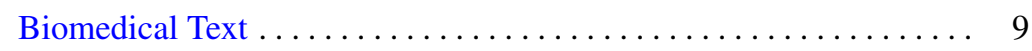

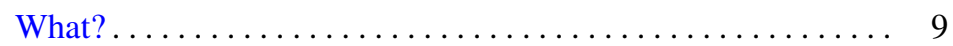

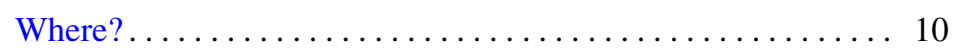

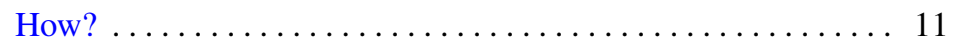

Semantics ............................... 11

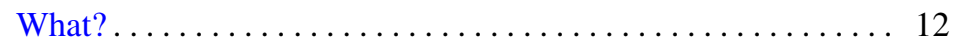

Where.$\ldots \ldots \ldots \ldots \ldots \ldots \ldots \ldots \ldots \ldots \ldots \ldots \ldots \ldots$

How? ............................... 14

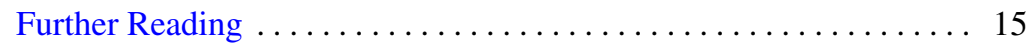

3 Data Retrieval ........................... 17

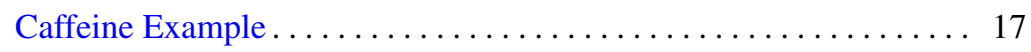

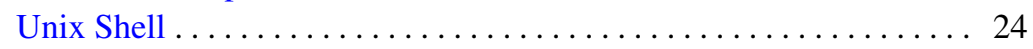

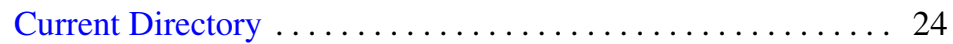

Windows Directories ........................ 25

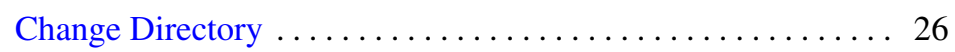

Useful Key Combinations ................... 26 


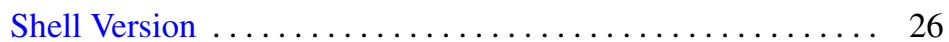

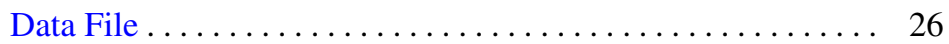

File Contents ........................... 27

Reverse File Contents ...................... 27

My First Script ........................ 27

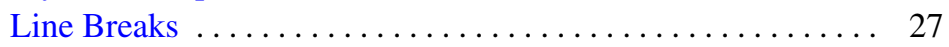

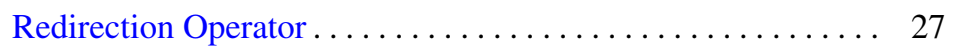

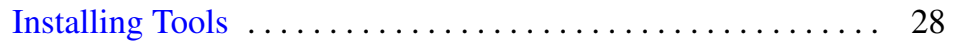

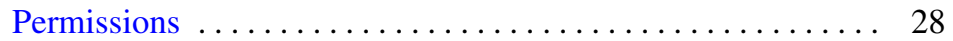

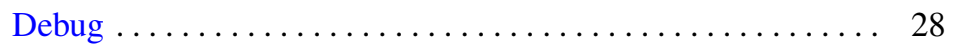

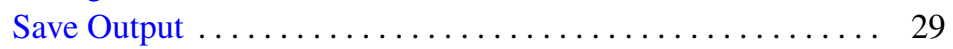

Web Identifiers ............................... 29

Single and Double Quotes $\ldots \ldots \ldots \ldots \ldots \ldots \ldots \ldots$

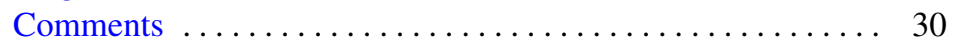

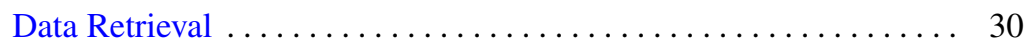

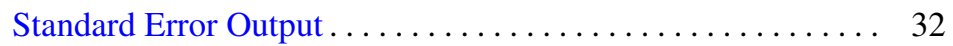

Data Extraction ................................. 32

Single and Multiple Patterns $\ldots \ldots \ldots \ldots \ldots \ldots \ldots \ldots \ldots$

Data Elements Selection ...................... 34

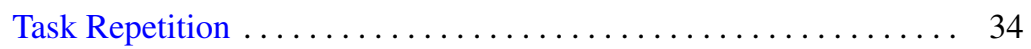

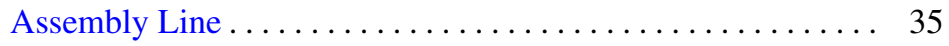

File Header . . . . . . . . . . . . . . . . . . . . . . . . . . . . 36

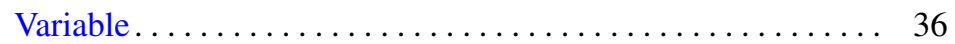

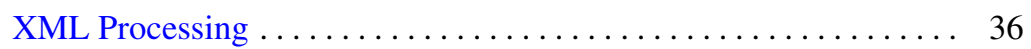

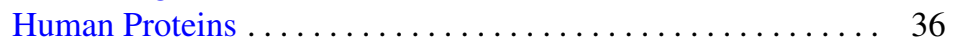

PubMed Identifiers $\ldots \ldots \ldots \ldots \ldots \ldots \ldots \ldots \ldots \ldots \ldots \ldots \ldots$

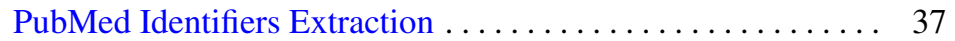

Duplicate Removal ......................... 38

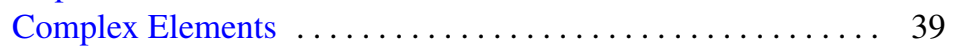

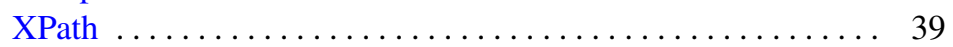

Namespace Problems $\ldots \ldots \ldots \ldots \ldots \ldots \ldots \ldots \ldots \ldots$

Only Local Names . . . . . . . . . . . . . . . . . . . . . . . . 39

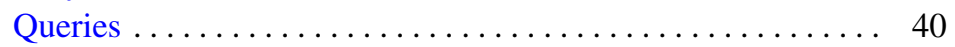

Extracting XPath Results $\ldots \ldots \ldots \ldots \ldots \ldots \ldots \ldots \ldots . \ldots 41$

Text Retrieval .............................. 41

Publication URL ......................... 41

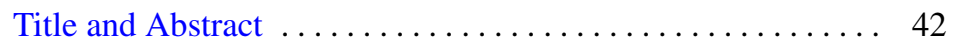

Disease Recognition ..................... 43

Further Reading. ......................... 43

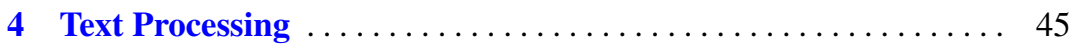

Pattern Matching ............................ 45

Case Insensitive Matching $\ldots \ldots \ldots \ldots \ldots \ldots \ldots \ldots \ldots$

Number of Matches ...................... 46

Invert Match ........................ 46

File Differences ............................. 46

Evaluation Metrics $\ldots \ldots \ldots \ldots \ldots \ldots \ldots \ldots \ldots \ldots \ldots 47$

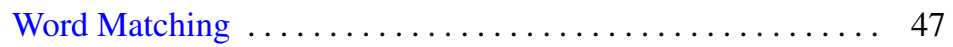


Regular Expressions $\ldots \ldots \ldots \ldots \ldots \ldots \ldots \ldots \ldots \ldots \ldots \ldots . \ldots \ldots$

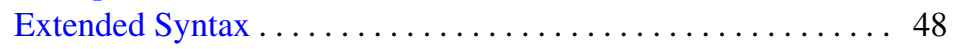

Alternation.......................... 49

Multiple Characters .......................... 49

Quantifiers .............................. 51

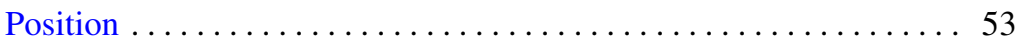

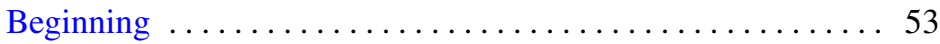

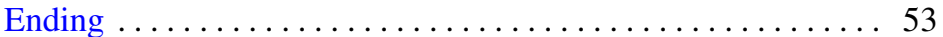

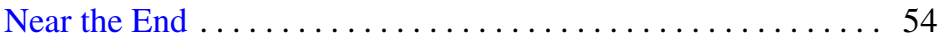

Word in Between ......................... 54

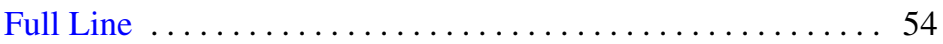

Match Position .......................... 55

Tokenization ............................... 55

Character Delimiters $\ldots \ldots \ldots \ldots \ldots \ldots \ldots \ldots \ldots \ldots$

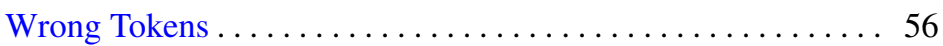

String Replacement $\ldots \ldots \ldots \ldots \ldots \ldots \ldots \ldots \ldots \ldots$

Multi-character Delimiters.................... 56

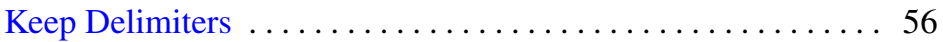

Sentences File ........................... 57

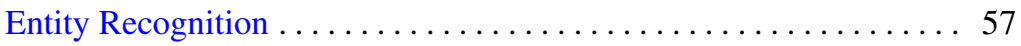

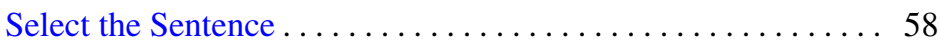

Pattern File ............................ 58

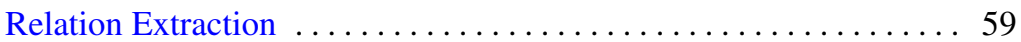

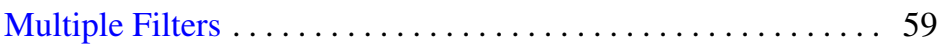

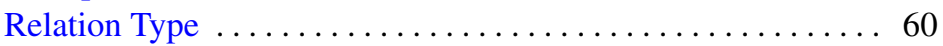

Remove Relation Types ..................... 60

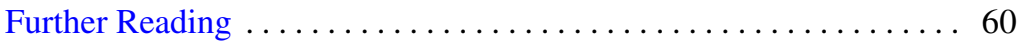

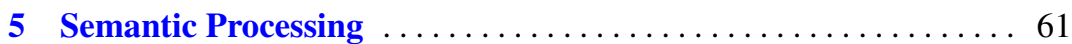

Classes.................................. 61

OWL Files . . . . . . . . . . . . . . . 61

Class Label ............................ 62

Class Definition ........................... 62

Related Classes ........................... 65

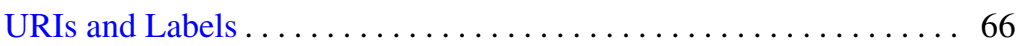

URI of a Label .......................... 66

Label of a URI ....................... 68

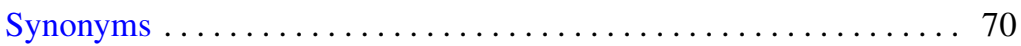

URI of Synonyms $\ldots \ldots \ldots \ldots \ldots \ldots \ldots \ldots \ldots \ldots \ldots \ldots \ldots \ldots$

Parent Classes . . . . . . . . . . . . . . . . . . . . 71

Labels of Parents ........................ 72

Related Classes ........................... 73

Labels of Related Classes . . . . . . . . . . . . . . . . . . 73

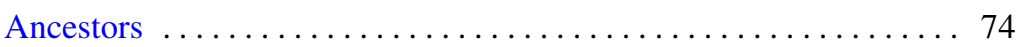

Grandparents ............................. 74

Root Class ............................... 74

Recursion................................ 74

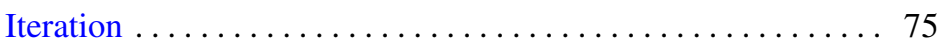




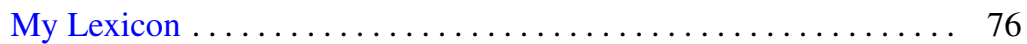

Ancestors Labels ......................... 76

Merging Labels ......................... 77

Ancestors Matched $\ldots \ldots \ldots \ldots \ldots \ldots \ldots \ldots \ldots \ldots \ldots$

Generic Lexicon .............................. 78

All Labels . . . . . . . . . . . . . . . . . . . . . . . . . 78

Problematic Entries . . . . . . . . . . . . . . . . . . . 79

Special Characters Frequency $\ldots \ldots \ldots \ldots \ldots \ldots \ldots \ldots$

Completeness .......................... 80

Removing Special Characters $\ldots \ldots \ldots \ldots \ldots \ldots \ldots \ldots$

Removing Extra Terms .................. 80

Removing Extra Spaces $\ldots \ldots \ldots \ldots \ldots \ldots \ldots \ldots \ldots$

Disease Recognition ...................... 81

Performance............................ 82

Inverted Recognition $\ldots \ldots \ldots \ldots \ldots \ldots \ldots \ldots \ldots \ldots \ldots \ldots$

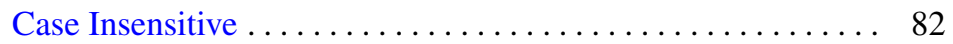

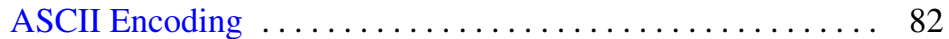

Correct Matches......................... 83

Incorrect Matches $\ldots \ldots \ldots \ldots \ldots \ldots \ldots \ldots \ldots \ldots \ldots$

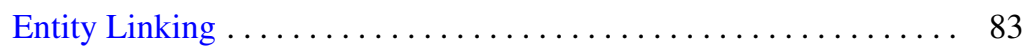

Modified Labels . . . . . . . . . . . . . . . . . . . . . . . . . . . . . 84

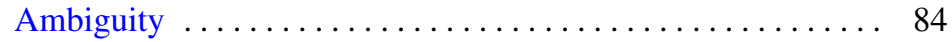

Surrounding Entities $\ldots \ldots \ldots \ldots \ldots \ldots \ldots \ldots \ldots \ldots$

Semantic Similarity ........................ 85

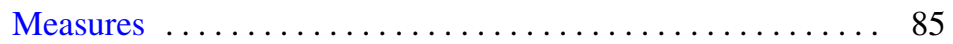

DiShIn Installation $\ldots \ldots \ldots \ldots \ldots \ldots \ldots \ldots \ldots \ldots \ldots$

Database File ................................ 87

DiShIn Execution ........................... 88

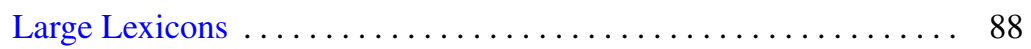

MER Installation $\ldots \ldots \ldots \ldots \ldots \ldots \ldots \ldots \ldots \ldots \ldots \ldots \ldots$

Lexicon Files ... . . . . . . . . . . . . . . . . . . . . . . . 89

MER Execution ........................... 90

Further Reading .......................... 91

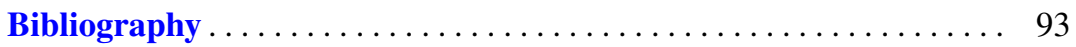

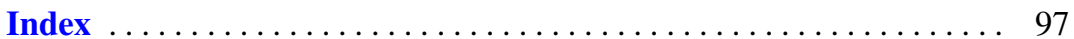




\section{Acronyms}

\begin{tabular}{ll} 
ChEBI & Chemical Entities of Biological Interest \\
CSV & Comma-Separated Values \\
cURL & Client Uniform Resource Locator \\
DAG & Directed Acyclic Graph \\
DBMS & Database Management System \\
DiShIn & Semantic Similarity Measures using Disjunctive Shared \\
& Information \\
DO & Disease Ontology \\
EBI & European Bioinformatics Institute \\
GO & Gene Ontology \\
HTTP & Hypertext Transfer Protocol \\
HTTPS & HTTP Secure \\
ICD & International Classification of Diseases \\
MER & Minimal Named-Entity Recognizer \\
MeSH & Medical Subject Headings \\
NCBI & National Center for Biotechnology Information \\
NER & Named-Entity Recognition \\
OBO & Open Biological and Biomedical Ontology \\
OWL & Web Ontology Language \\
PMC & PubMed Central \\
RDFS & RDF Schema \\
SNOMED CT & Systematized Nomenclature of Medicine - Clinical Terms \\
SQL & Structured Query Language \\
TSV & Tab-Separated Values \\
UMLS & Unified Medical Language System \\
UniProt & Universal Protein Resource \\
URI & Uniform Resource Identifier \\
URL & Uniform Resource Locator \\
XLS & Microsoft Excel file format \\
XML & Extensible Markup Language \\
& \\
\hline &
\end{tabular}




\section{Introduction}

\begin{abstract}
Health and Life studies are well known for the huge amount of data they produce, such as high-throughput sequencing projects (Stephens et al., PLoS Biol 13(7):e1002195, 2015; Hey et al., The fourth paradigm: data-intensive scientific discovery, vol 1 . Microsoft research Redmond, Redmond, 2009). However, the value of the data should not be measured by its amount, but instead by the possibility and ability of researchers to retrieve and process it (Leonelli, Data-centric biology: a philosophical study. University of Chicago Press, Chicago, 2016). Transparency, openness, and reproducibility are key aspects to boost the discovery of novel insights into how living systems work (Nosek et al., Science 348(6242):1422-1425, 2015).
\end{abstract}

\section{Keywords}

Bioinformatics - Biomedical data repositories - Text files · EBI: European Bioinformatics Institute $\cdot$ Bibliographic databases $\cdot$ Shell scripting $\cdot$ Command line tools $\cdot$ Spreadsheet applications $\cdot$ CSV: comma-separated values - TSV: tab-separated values

\section{Biomedical Data Repositories}

Fortunately, a significant portion of the biomedical data is already being collected, integrated and distributed through Biomedical Data Repositories, such as European Bioinformatics Institute (EBI) and National Center for Biotechnology Information (NCBI) repositories (Cook et al. 2017; Coordinators 2018). Nonetheless, researchers cannot rely on available data as mere facts, they may contain errors, can be outdated, and may require a context (Ferreira et al. 2017). Most facts are only valid in a specific biological setting and should not be directly extrapolated to other cases. In addition, different research communities have different needs and requirements, which change over time (Tomczak et al. 2018).

\section{Scientific Text}

Structured data is what most computer applications require as input, but humans tend to prefer the flexibility of text to express their hypothesis, ideas, opinions, conclusions (Barros and Couto 2016). This explains why scientific text is still the preferential means to publish new 
discoveries and to describe the data that support them (Holzinger et al. 2014; Lu 2011). Another reason is the long-established scientific reward system based on the publication of scientific articles (Rawat and Meena 2014).

\section{Amount of Text}

The main problem of analyzing biomedical text is the huge amount of text being published every day (Hersh 2008). For example, 813,598 citations ${ }^{1}$ were added in 2017 to MEDLINE, a bibliographic database of Health and Life literature ${ }^{2}$. If we read 10 articles per day, it will take us takes more than 222 years to just read those articles. Figure 1.1 presents the number of citations added to MEDLINE in the past decades, showing the increasing large amount of biomedical text that researchers must deal with.

Moreover, scientific articles are not the only source of biomedical text, for example clinical studies and patents also provide a large amount of text to explore. They are also growing at a fast pace, as Figs. 1.2 and 1.3 clearly show (Aras et al. 2014; Jensen et al. 2012).

\section{Ambiguity and Contextualization}

Given the high flexibility and ambiguity of natural language, processing and extracting information from texts is a painful and hard task, even to humans. The problem is even more complex when dealing with scientific text, that requires specialized expertise to understand it. The major problem with Health and Life Sciences is the inconsistency of the nomenclature used for describing biomedical concepts and entities (Hunter and Cohen 2006; Rebholz-Schuhmann et al. 2005). In biomedical text, we can often find different terms referring to the same biological concept or entity (synonyms), or the same term meaning different

\footnotetext{
${ }^{1}$ https://www.nlm.nih.gov/bsd/index_stats_comp.html

${ }^{2}$ https://www.nlm.nih.gov/bsd/medline.html
}

biological concepts or entities (homonyms). For example, many times authors improve the readability of their publications by using acronyms to mention entities, that may be clear for experts on the field but ambiguous in another context.

The second problem is the complexity of the message. Almost everyone can read and understand a newspaper story, but just a few can really understand a scientific article. Understanding the underlying message in such articles normally requires years of training to create in our brain a semantic model about the domain and to know how to interpret the highly specialized terminology specific to each domain. Finally, the multilingual aspect of text is also a problem, since most clinical data are produced in the native language (Campos et al. 2017).

\section{Biomedical Ontologies}

To address the issue of ambiguity of natural language and contextualization of the message, text processing techniques can explore current biomedical ontologies (Robinson and Bauer 2011). These ontologies can work as vocabularies to guide us in what to look for (Couto et al. 2006). For example, we can select an ontology that models a given domain and find out which official names and synonyms are used to mention concepts in which we have an interest (Spasic et al. 2005). Ontologies may also be explored as semantic models by providing semantic relationships between concepts (Lamurias et al. 2017).

\section{Programming Skills}

The success of biomedical studies relies on overcoming data and text processing issues to take the most of all the information available in biomedical data repositories. In most cases, biomedical data analysis is no longer possible using an inhouse and limited dataset, we must be able to efficiently process all this data and text. So, a common question that many Health and Life specialists face is: 


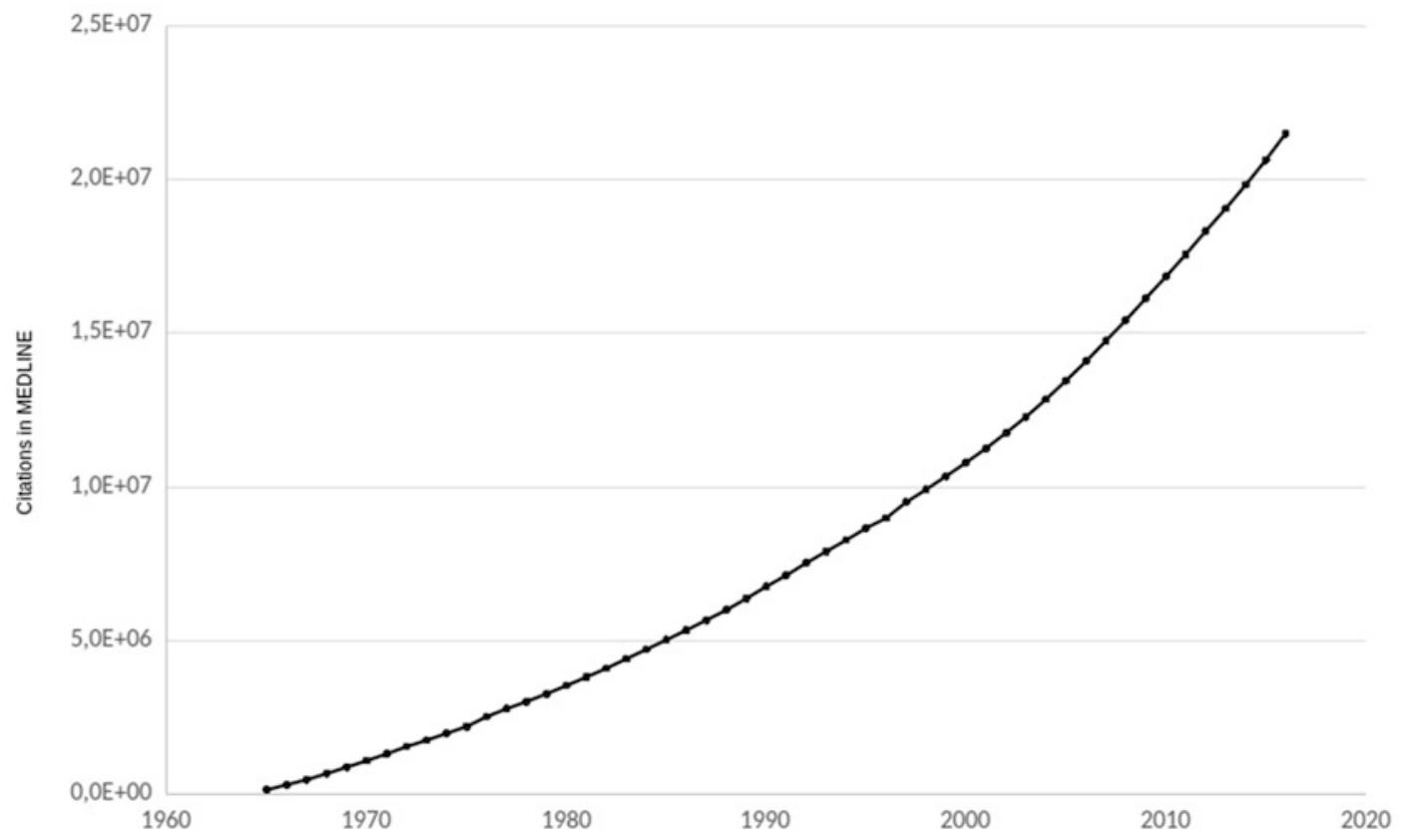

Fig. 1.1 Chronological listing of the total number of citations in MEDLINE (Source: https://www.nlm.nih.gov/bsd/)

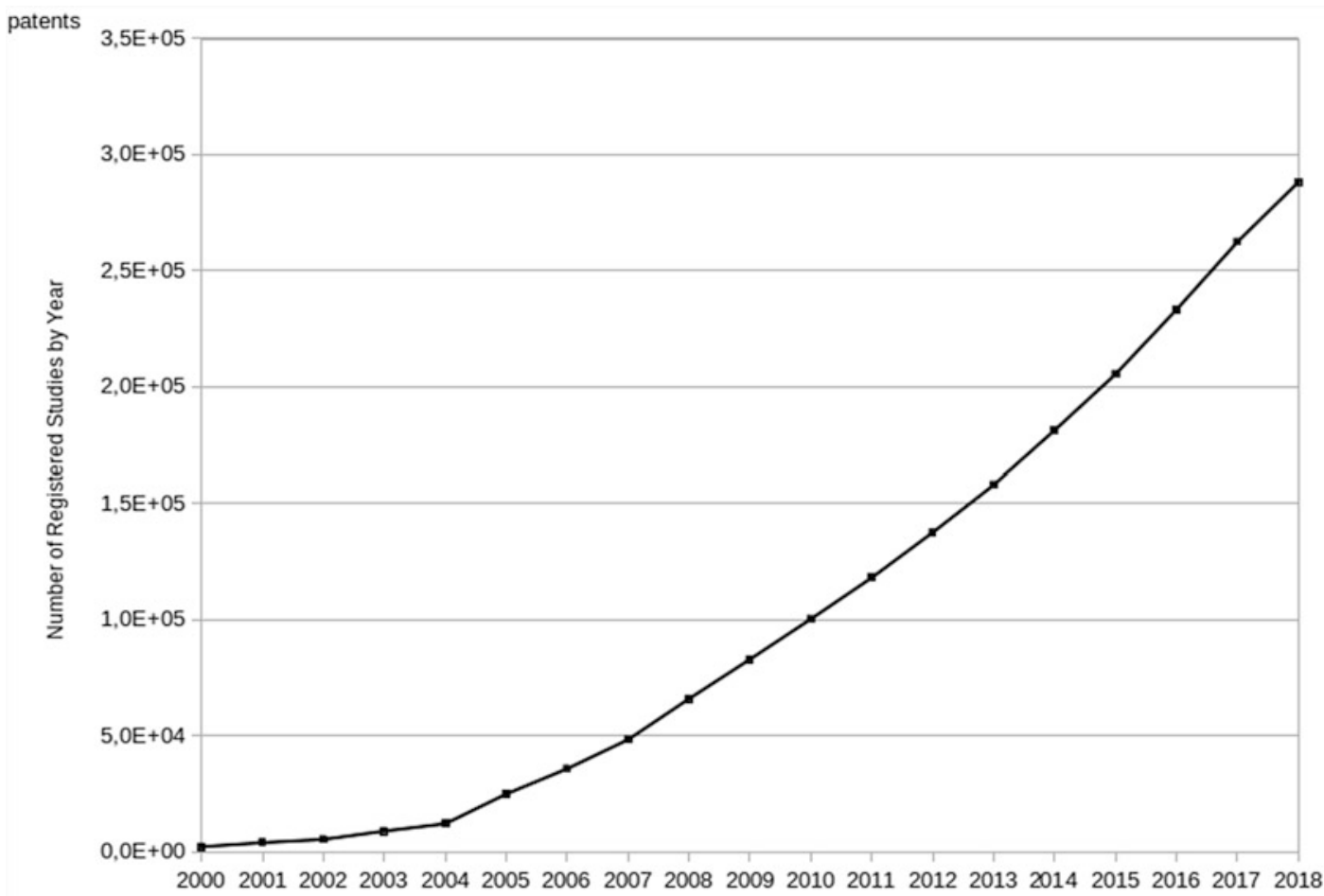

Fig. 1.2 Chronological listing of the total number of registered studies (clinical trials) (Source: https://clinicaltrials. gov) 


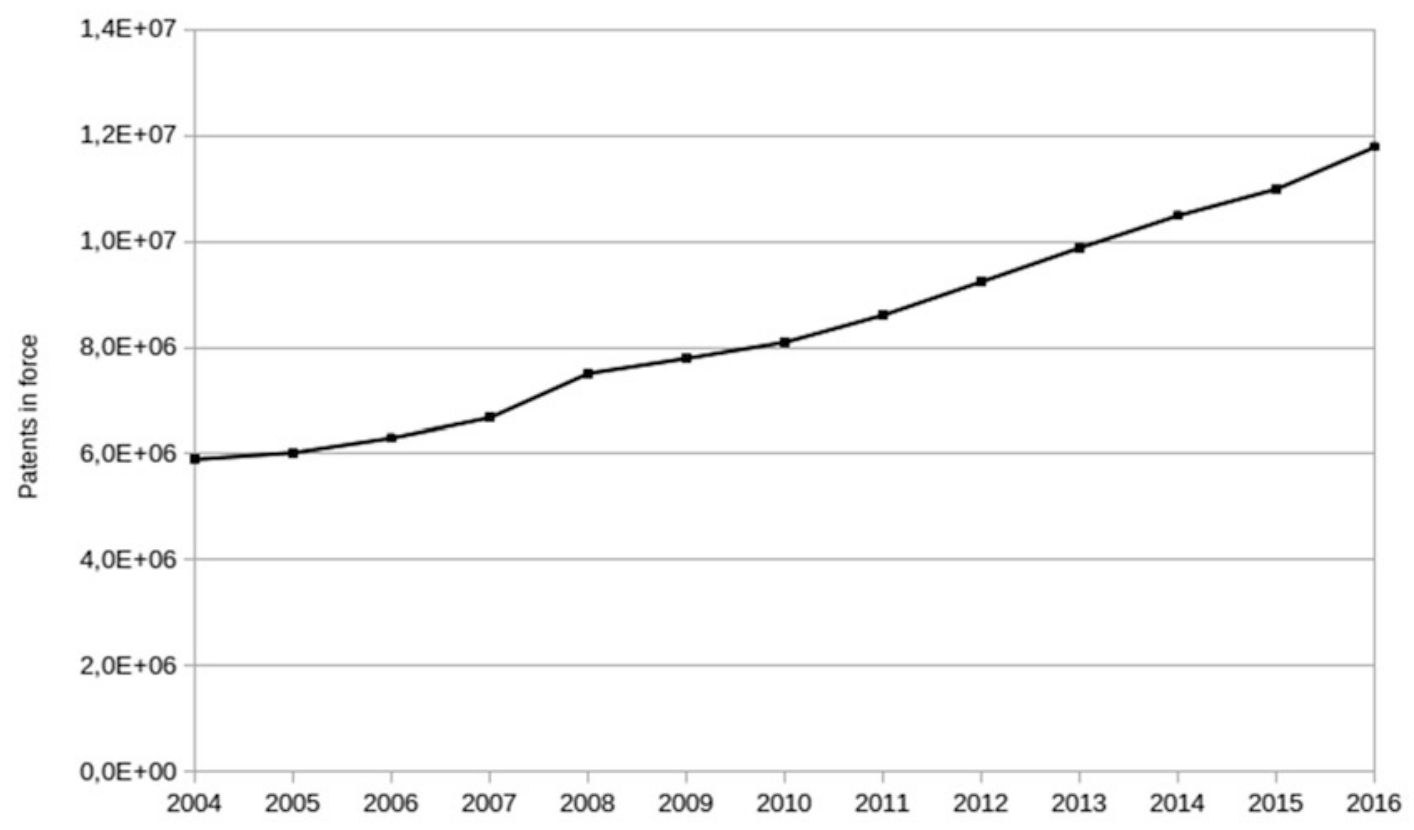

Fig. 1.3 Chronological listing of the total number of patents in force (Source: WIPO statistics database http://www. wipo.int/ipstats/en/)

How can I deal with such huge amount of data and text the necessary expertise, time and disposition to learn computer programming?

This is the goal of this book, to provide a lowcost, long-lasting, feasible and painless answer to this question.

\section{Why This Book?}

State-of-the-art data and text processing tools are nowadays based on complex and sophisticated technologies, and to understand them we need to have special knowledge on programming, linguistics, machine learning or deep learning (Holzinger and Jurisica 2014; Ching et al. 2018; Angermueller et al. 2016). Explaining their technicalities or providing a comprehensive list of them are not the purpose of this book. The tools implementing these technologies tend to be impenetrable to the common Health and Life specialists and usually become outdated or even unavailable some time after their publication or the financial support ends. Instead, this book will equip the reader with a set of skills to process text with minimal dependencies to existing tools and technologies. The idea is not to explain how to build the most advanced tool, but how to create a resilient and versatile solution with acceptable results.

In many cases, advanced tools may not be most efficient approach to tackle a specific problem. It all depends on the complexity of problem, and the results we need to obtain. Like a good physician knows that the most efficient treatment for a specific patient is not always the most advanced one, a good data scientist knows that the most efficient tool to address a specific information need is not always the most advanced one. Even without focusing on the foundational basis of programming, linguistics or artificial intelligence, this book provides the basic knowledge and right references to pursue a more advanced solution if required. 


\section{Third-Party Solutions}

Many manuscripts already present and discuss the most recent and efficient text mining techniques and the available software solutions based on them that users can use to process data and text (Cock et al. 2009; Gentleman et al. 2004; Stajich et al. 2002). These solutions include stand-alone applications, web applications, frameworks, packages, pipelines, etc. A common problem with these solutions is their resiliency to deal with new user requirements, to changes on how resources are being distributed, and to software and hardware updates. Commercial solutions tend to be more resilient if they have enough customers to support the adaptation process. But of course we need the funding to buy the service. Moreover, we will be still dependent on a third-party availability to address our requirements that are continuously changing, which vary according to the size of the company and our relevance as client.

Using open-source solutions may seem a great alternative since we do not need to allocate funding to use the service and its maintenance is assured by the community. However, many of these solutions derive from academic projects that most of the times are highly active during the funding period and then fade away to minimal updates. The focus of academic research is on creating new and more efficient methods and publish them, the software is normally just a means to demonstrate their breakthroughs. In many cases to execute the legacy software is already a nontrivial task, and even harder is to implement the required changes. Thus, frequently the most feasible solution is to start from scratch.

\section{Simple Pipelines}

If we are interested in learning sophisticated and advanced programming skills, this is not the right book to read. This book aims at helping Health and Life specialists to process data and text by describing a simple pipeline that can be executed with minimal software dependencies. Instead of using a fancy web front-end, we can still man- ually manipulate our data using the spreadsheet application that we already are comfortable with, and at the same time be able to automatize some of the repetitive tasks.

In summary, this book is directed mainly towards Health and Life specialists and students that need to know how to process biomedical data and text, without being dependent on continuous financial support, third-party applications, or advanced computer skills.

\section{How This Book Helps Health and Life Specialists?}

So, if this book does not focus on learning programming skills, and neither on the usage of any special package or software, how it will help specialists processing biomedical text and data?

\section{Shell Scripting}

The solution proposed in this book has been available for more than four decades (Ritchie 1971), and it can now be used in almost every personal computer (Haines 2017). The idea is to provide an example driven introduction to shell scripting ${ }^{3}$ that addresses common challenges in biomedical text processing using a Unix shell ${ }^{4}$. Shells are software programs available in Unix operating systems since $1971^{5}$, but nowadays are available is most of our personal computers using Linux, macOS or Windows operating systems.

But a shell script is still a computer algorithm, so how is it different from learning another programming language?

\footnotetext{
${ }^{3}$ https://en.wikipedia.org/wiki/Shell_script

${ }^{4}$ https://en.wikipedia.org/wiki/Unix_shell

${ }^{5}$ https://www.in-ulm.de/ mascheck/bourne/\#origins
} 
It is different in the sense that most solutions are based on the usage of single command line tools, that sometimes are combined as simple pipelines. This book does not intend to create experts in shell scripting, by the contrary, the few scripts introduced are merely direct combinations of simple command line tools individually explained before.

The main idea is to demonstrate the ability of a few command line tools to automate many of the text and data processing tasks. The solutions are presented in a way that comprehending them is like conducting a new laboratory protocol i.e. testing and understanding its multiple procedural steps, variables, and intermediate results.

\section{Text Files}

All the data will be stored in text files, which command line tools are able to efficiently process (Baker and Milligan 2014). Text files represent a simple and universal medium of storing our data. They do not require any special encoding and can be opened and interpreted by using any text editor application. Normally, text files without any kind of formatting are stored using a $t x t$ extension. However, text files can contain data using a specific format, such as:

CSV : Comma-Separated Values ${ }^{6}$;

TSV : Tab-Separated Values ${ }^{7}$;

XML : eXtensible Markup Language ${ }^{8}$.

All the above formats can be open (import), edited and saved (export) by any text editor application. and common spreadsheet applications ${ }^{9}$, such as LibreOffice Calc or Microsoft Excel ${ }^{10}$. For example, we can create a new data file using LibreOffice Calc, like the one in Fig. 1.4. Then we select the option to save it as CSV, TSV, XML

\footnotetext{
${ }^{6}$ https://en.wikipedia.org/wiki/Comma-separated_values ${ }^{7}$ https://en.wikipedia.org/wiki/Tab-separated_values

${ }^{8}$ https://en.wikipedia.org/wiki/XML

${ }^{9}$ https://en.wikipedia.org/wiki/Spreadsheet

${ }^{10}$ To save in TSV format using the LibreOffice Calc, we may have to choose CSV format and then select as field delimiter the tab character.
}

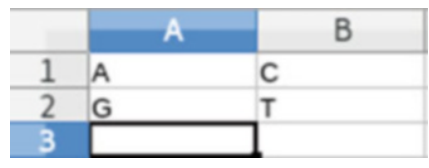

Fig. 1.4 Spreadsheet example

(Microsoft 2003), and XLS (Microsoft 2003) formats. We can try to open all these files in our favorite text editor.

When opening the CSV file, the application will show the following contents:

\section{$A, C$}

$\mathrm{G}, \mathrm{T}$

Each line represents a row of the spreadsheet, and column values are separated by commas.

When opening the TSV file, the application will show the following contents:

$\begin{array}{ll}\text { A } & \text { C } \\ \text { G } & \text { T }\end{array}$

The only difference is that instead of a comma it is now used a tab character to separate column values.

When opening the XML file, the application will show the following contents:

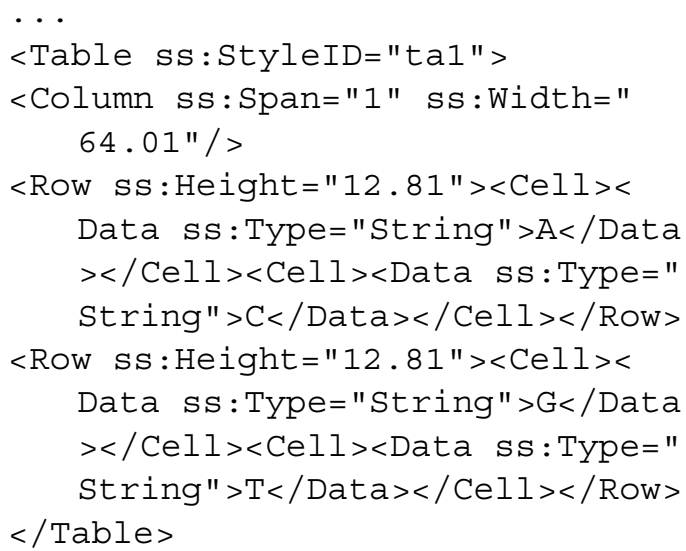

Now the data is more complex to find and understand, but with a little more effort we can check that we have a table with two rows, each one with two cells.

When opening the XLS file, we will get a lot of strange characters and it is humanly impossible to understand what data it is storing. 
This happens because XLS is not a text file is a proprietary format ${ }^{11}$, which organizes data using an exclusive encoding scheme, so its interpretation and manipulation could only be done using a specific software application.

Comma-separated values is a data format so old as shell scripting, in 1972 it was already supported by an IBM product ${ }^{12}$. Using CSV or TSV enables us to manually manipulate the data using our favorite spreadsheet application, and at the same time use command line tools to automate some of the tasks.

\section{Relational Databases}

If there is a need to use more advanced data storage techniques, such as using a relational database $^{13}$, we may still be able to use shell scripting if we can import and export our data to a text format. For example, we can open a relational database, execute Structured Query Language (SQL) commands ${ }^{14}$, and import and export the data to CSV using the command line tool sqlite $3^{15}$.

Besides CSV and shell scripting being almost the same as they were four decades ago, they are still available everywhere and are able to solve most of our data and text processing daily problems. So, these tools are expected to continue to be used for many more decades to come. As a bonus, we will look like a true professional typing command line instructions in a black background window $! \ddot{*}$

\section{What Is in the Book?}

First, the Chap. 2 presents a brief overview of some of the most prominent resources of biomedical data, text, and semantics. The chapter dis-

\footnotetext{
${ }^{11}$ https://en.wikipedia.org/wiki/Proprietary_format

${ }^{12}$ http://bitsavers.trailingedge.com/pdf/ibm/370/fortran/ GC28-6884-0_IBM_FORTRAN_Program_Products_for_ OS_and_CMS_General_Information_Jul72.pdf

${ }^{13}$ https://en.wikipedia.org/wiki/Relational_database

${ }^{14}$ https://en.wikipedia.org/wiki/SQL

${ }^{15}$ https://www.sqlite.org/cli.html
}

cusses what type of information they distribute, where we can find them, and how we will be able to automatically explore them. Most of the examples in the book use the resources provided by the European Bioinformatics Institute (EBI) and use their services to automatically retrieve the data and text. Nevertheless, after understanding the command line tools, it will not be hard to adapt them to the formats used by other service provider, such as the National Center for Biotechnology Information (NCBI). In terms of semantics, the examples will use two ontologies, one about human diseases and the other about chemical entities of biological interest. Most ontologies share the same structure and syntax, so adapting the solutions to other domains are expected to be painless.

As an example, the Chap. 3 will describe the manual steps that Health and Life specialists may have to perform to find and retrieve biomedical text about caffeine using publicly available resources. Afterwards, these manual steps will be automatized by using command line tools, including the automatic download of data. The idea is to go step-by-step and introduce how each command line tool can be used to automate each task.

\section{Command Line Tools}

The main command line tools that this book will introduce are the following:

- curl: a tool to download data and text from the web;

- grep: a tool to search our data and text;

- gawk: a tool to manipulate our data and text;

- sed: a tool to edit our data and text;

- xargs: a tool to repeat the same step for multiple data items;

- xmllint: a tool to search in XML data files.

Other command line tools are also presented to perform minor data and text manipulations, such as:

- cat: a tool to get the content of file; 
- tr: a tool to replace one character by another;

- sort: a tool to sort multiple lines;

- head: a tool to select only the first lines.

\section{Pipelines}

A fundamental technique introduced in Chap. 3 is how to redirect the output of a command line tool as input to another tool, or to a file. This enables the construction of pipelines of sequential invocations of command line tools. Using a few commands integrated in a pipeline is really the maximum shell scripting that this book will use. Scripts longer than that would cross the line of not having to learn programming skills.

Chapter 4 is about extracting useful information from the text retrieved previously. The example consists in finding references to malignant hyperthermia in these caffeine related texts, so we may be able to check any valid relation.

\section{Regular Expressions}

A powerful pattern matching technique described in this chapter is the usage of regular expressions $^{16}$ in the grep command line tool to perform Named-Entity Recognition (NER) ${ }^{17}$. Regular expressions originated in 1951 (Kleene 1951), so they are even older than shell scripting, but still popular and available in multiple software applications and programming languages (Forta
2018). A regular expression is a string that include special operators represented by special characters. For example, the regular expression $A|C| G \mid T$ will identify in a given string any of the four nucleobases adenine (A), cytosine (C), guanine $(\mathrm{G})$, or thymine $(\mathrm{T})$.

Another technique introduced is tokenization. It addresses the challenge of identifying the text boundaries, such as splitting a text into sentences. So, we can keep only the sentences that may have something we want. Chapter 4 also describes how can we try to find two entities in the same sentence, providing a simple solution to the relation extraction challenge ${ }^{18}$.

\section{Semantics}

Instead of trying to recognize a limited list of entities, Chap. 5 explains how can we use ontologies to construct large lexicons that include all the entities of a given domain, e.g. humans diseases. The chapter also explains how the semantics encoded in an ontology can be used to expand a search by adding the ancestors and related classes of a given entity. Finally, a simple solution to the Entity Linking ${ }^{19}$ challenge is given, where each entity recognized is mapped to a class in an ontology. A simple technique to solve the ambiguity issue when the same label can be mapped to more than one class is also briefly presented.

\footnotetext{
${ }^{16}$ https://en.wikipedia.org/wiki/Regular_expression

${ }^{17}$ https://en.wikipedia.org/wiki/Named-

entity_recognition
}

\footnotetext{
${ }^{18}$ https://en.wikipedia.org/wiki/Relationship_extraction

${ }^{19}$ https://en.wikipedia.org/wiki/Entity_linking
}

Open Access This chapter is licensed under the terms of the Creative Commons Attribution 4.0 International License (http://creativecommons.org/licenses/by/4.0/), which permits use, sharing, adaptation, distribution and reproduction in any medium or format, as long as you give appropriate credit to the original author(s) and the source, provide a link to the Creative Commons licence and indicate if changes were made.

The images or other third party material in this chapter are included in the chapter's Creative Commons licence, unless indicated otherwise in a credit line to the material. If material is not included in the chapter's Creative Commons licence and your intended use is not permitted by statutory regulation or exceeds the permitted use, you will need to obtain permission directly from the copyright holder. 


\section{Abstract}

The previous chapter presented the importance of text and semantic resources for Health and Life studies. This chapter will describe what kind of text and semantic resources are available, where they can be found, and how they can be accessed and retrieved.

\section{Keywords}

Biomedical literature - Programmatic access . UniProt citations service $\cdot$ Semantics .

Controlled vocabularies - Ontologies - OWL: Web Ontology Language · URI: Uniform Resource Identifier · DAG: Directed Acyclic Graphs · OBO: Open Biomedical Ontologies

\section{Biomedical Text}

Text is still the preferential means of publishing novel knowledge in Health and Life Sciences, and where we can expect to find all the information about the supporting data. Text can be found and explored in multiple types of sources, the main being scientific articles and patents (Krallinger et al. 2017). However, less formal texts are also relevant to explore, such as the ones present nowadays in electronic health records (Blumenthal and Tavenner 2010).

\section{What?}

In the biomedical domain, we can find text in different forms, such as:

Statement: a short piece of text, normally containing personal remarks or an evidence about a biomedical phenomenon;

Abstract: a short summary of a larger scientific document;

Full-text: the entire text present in a scientific document including scattered text such as figure labels and footnotes.

Statements contain more syntactic and semantic errors than abstracts, since they normally are not peer-reviewed, but they are normally directly linked to data providing useful details about it. The main advantage of using statements or abstracts is the brief and succinct form on which the information is expressed. In the case of abstracts, there was already an intellectual exercise to present only the main facts and ideas. Nevertheless, a brief description may be insufficient to draw a solid conclusion, that may require some important details not possible to summarize in a short piece of text (Schuemie et al. 2004). These details are normally presented in the form of a full-text document, which contains a complete description of the results obtained. For example, important details are sometimes only present in figure labels (Yeh et al. 2003). 
One major problem of full-text documents is their availability, since their content may have restricted access. In addition, the structure of the full-text and the format on which is available varies according to the journal in where it was published. Having more information does not mean that all of it is beneficial to find what we need. Some of the information may even induce us in error. For example, the relevance of a fact reported in the Results Section may be different if the fact was reported in the Related Work Section. Thus, the usage of full-text may create several problems regarding the quality of information extracted (Shah et al. 2003).

\section{Where?}

Access to biomedical literature is normally done using the internet through PubMed ${ }^{1}$, an information retrieval system released in 1996 that allows researchers to search and find biomedical texts of relevance to their studies (Canese 2006). PubMed is developed and maintained by the National Center for Biotechnology Information (NCBI), at the U.S. National Library of Medicine (NLM), located at the National Institutes of Health (NIH). Currently, PubMed provides access to more than 28 million citations from MEDLINE, a bibliographic database with references to a comprehensive list of academic journals in Health and Life Sciences ${ }^{2}$. The references include multiple metadata about the documents, such as: title, abstract, authors, journal, publication date. PubMed does not store the full-text documents, but it provides links where we may find the full-text. More recently, biomedical references are also accessible using the European Bioinformatics Institute (EBI) services, such as Europe $\mathrm{PMC}^{3}$, the Universal Protein Resource (UniProt) with its UniProt citations service ${ }^{4}$.

Other generic alternative tools have been also gaining popularity for finding scientific texts,

\footnotetext{
${ }^{1}$ https://www.nlm.nih.gov/bsd/pubmed.html

${ }^{2} \mathrm{https}: / /$ www.nlm.nih.gov/bsd/medline.html

${ }^{3}$ http://europepmc.org/

${ }^{4}$ https://www.uniprot.org/citations/
}

such as Google Scholar ${ }^{5}$, Google Patents ${ }^{6}$, ResearchGate $^{7}$ and Mendeley ${ }^{8}$.

More than just text some tools also integrate semantic links. One of the first search engines for biomedical literature to incorporate semantics was GOPubMed ${ }^{9}$, that categorized texts according to Gene Ontology terms found in them (Doms and Schroeder 2005). These semantic resources will be described in a following section. A more recent tool is PubTator ${ }^{10}$ that provides the text annotated with biological entities generated by state-of-the-art text-mining approaches (Wei et al. 2013).

There is also a movement in the scientific community to produce Open Access Publications, making full-texts freely available with unrestricted use. One of the main free digital archives of free biomedical full-texts is PubMed Central $^{11}$ (PMC), currently providing access to more than 5 million documents.

Other relevant source of biomedical texts is the electronic health records stored in health institutions, but the texts they contain are normally directly linked to patients and therefore their access is restricted due to ethical and privacy issues. As example, the THYME corpus ${ }^{12}$ includes more than one thousand de-identified clinical notes from the Mayo Clinic, but is only available for text processing research under a data use agreement (DUA) with Mayo Clinic (Styler IV et al. 2014).

From generic texts we can also sometimes find relevant biomedical information. For example, some recent biomedical studies have been processing the texts in social networks to identify new trends and insights about a disease, such as processing tweets to predict flu outbreaks (Aramaki et al. 2011).

\footnotetext{
${ }^{5}$ http://scholar.google.com/

${ }^{6}$ http://www.google.com/patents

${ }^{7}$ https://www.researchgate.net/

${ }^{8}$ https://www.mendeley.com/

${ }^{9}$ https://gopubmed.org/

${ }^{10}$ http://www.ncbi.nlm.nih.gov/CBBresearch/Lu/Demo/ PubTator/

${ }^{11}$ https://www.ncbi.nlm.nih.gov/pmc/

${ }^{12} \mathrm{http}: / /$ thyme.healthnlp.org/
} 


\section{How?}

To automatically process text, we need programmatic access to it, this means that from the previous biomedical data repositories we can only use the ones that allow this kind of access. These limitations are imposed because many biomedical documents have copyright restrictions hold by their publishers. And some restrictions may define that only manual access is granted, and no programmatic access is allowed. These restrictions are normally detailed in the terms of service of each repository. However, when browsing the repository if we face a CAPTCHA challenge to determine whether we are humans or not, probably means that some access restrictions are in place.

Fortunately, $\mathrm{NCBI}^{13}$ and $\mathrm{EBI}^{14}$ online services, such as PubMed, Europe PMC, or UniProt Citations, allow programmatic access ( $\mathrm{Li}$ et al. 2015). Both institutions provide Web APIs ${ }^{15}$ that fully document how web services can be programmatically invoked. Some resources can inclusively be accessed using RESTful web services ${ }^{16}$ that are characterized by a simple uniform interface that make any Uniform Resource Locator (URL) almost self-explanatory (Richardson and Ruby 2008). The same URL shown by our web browser is the only thing we need to know to retrieve the data using a command line tool.

For example, if we search for caffeine using the UniProt Citations service ${ }^{17}$, select the first two entries, and click on download, the browser will show information about those two documents using a tabular format.

\section{PubMed ID Title Authors/Groups Abstract/Summary \\ 27702941 Genome-wide association ... \\ 22333316 Modeling caffeine concentrations ...}

\footnotetext{
${ }^{13}$ https://www.ncbi.nlm.nih.gov/home/develop/api/

${ }^{14}$ https://www.ebi.ac.uk/seqdb/confluence/display/ JDSAT/

${ }^{15} \mathrm{https} / / /$ en.wikipedia.org/wiki/Web_API

${ }^{16}$ https://www.ebi.ac.uk/seqdb/confluence/pages/ viewpage .action?pageId=68165098

${ }^{17}$ https://www.uniprot.org/citations/
}

More important is to check the URL that is now being used:

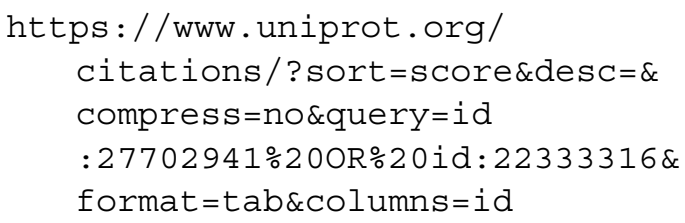

We can check that the URL has three main components: the scheme (https), the hostname (www.uniprot.org), the service (citations) and the data parameters. The scheme represents the type of web connection to get the data, and usually is one of these protocols: Hypertext Transfer Protocol (HTTP) or HTTP Secure (HTTPS) ${ }^{18}$. The hostname represents the physical site where the service is available. The list of parameters depends on the data available from the different services. We can change any value of the parameters (arguments) to get different results. For example, we can replace the two PubMed identifiers by the following one $29029291^{19}$, and our browser will now display the information about this new document:

\section{PubMed ID Title Authors/Groups \\ Abstract/Summary \\ 29029291 Nutrition Influences...}

The good news is that we can use this link with a command line tool and automatize the retrieval of the data, including extracting the abstract to process its text.

\section{Semantics}

Lack of use of standard nomenclatures across biological text makes text processing a non-trivial task. Often, we can find different labels (synonyms, acronyms) for the same biomedical entities, or, even more problematic, different entities sharing the same label (homonyms) (RebholzSchuhmann et al. 2005). Sense disambiguation to select the correct meaning of an expression in

\footnotetext{
${ }^{18} \mathrm{https} / / /$ en.wikipedia.org/wiki/ Hypertext_Transfer_Protocol

${ }^{19} \mathrm{https}: / /$ www.uniprot.org/citations/?sort=score $\&$ desc $=$ $\&$ compress=no\&query=id:29029291\&format= tab\&columns=id
} 
a given piece of text is therefore a crucial issue. For example, if we find the disease acronym ATS in a text, we may have to figure out if it representing the Andersen-Tawil syndrome ${ }^{20}$ or the $X$ linked Alport syndrome ${ }^{21}$. Further in the book, we will address this issue by using ontologies and semantic similarity between their classes (Couto and Lamurias 2019).

\section{What?}

In 1993, Gruber (1993) proposed a short but comprehensive definition of ontology as an:

an explicit specification of a conceptualization

In 1997 and 1998, Borst and Borst (1997) and Studer et al. (1998) refined this definition to:

a formal, explicit specification of a shared conceptualization

A conceptualization is an abstract view of the concepts and the relationships of a given domain. A shared conceptualization means that a group of individuals agree on that view, normally established by a common agreement among the members of a community. The specification is a representation of that conceptualization using a given language. The language needs to be formal and explicit, so computers can deal with it.

\section{Languages}

The Web Ontology Language $(\mathrm{OWL})^{22}$ is nowadays becoming one of the most common languages to specify biomedical ontologies (McGuinness et al. 2004). Another popular alternative is the Open Biomedical Ontology $(\mathrm{OBO})^{23}$ format developed by the OBO foundry. OBO established a set of principles to ensure high quality, formal rigor and interoperability between other OBO ontologies (Smith et al. 2007). One important principle is that $\mathrm{OBO}$ ontologies need

\footnotetext{
${ }^{20}$ http://purl.obolibrary.org/obo/DOID_0050434

${ }^{21}$ http://purl.obolibrary.org/obo/DOID_0110034

${ }^{22} \mathrm{https}$ ///en.wikipedia.org/wiki/

Web_Ontology_Language

${ }^{23}$ https://en.wikipedia.org/wiki/

Open_Biomedical_Ontologies
}

to be open and available without any constraint other than acknowledging their origin.

Concepts are defined as OWL classes that may include multiple properties. For text processing important properties include the labels that may be used to mention that class. The labels may include the official name, acronyms, exact synonyms, and even related terms. For example, a class defining the disease malignant hyperthermia may include as synonym anesthesia related hyperthermia. Two distinct classes may share the same label, such as Andersen-Tawil syndrome and X-linked Alport syndrome that have ATS as an exact synonym.

\section{Formality}

The representation of classes and the relationships may use different levels of formality, such as controlled vocabularies, taxonomies and thesaurus, that even may include logical axioms.

Controlled vocabularies are list of terms without specifying any relation between them. Taxonomies are controlled vocabularies that include subsumption relations, for example specifying that malignant hyperthermia is a muscle tissue disease. This is- $a$ or subclass relations are normally the backbone of ontologies. We should note that some ontologies may include multiple inheritance, i.e. the same concept may be a specialization of two different concepts. Therefore, many ontologies are organized as a directed acyclic graphs (DAG) and not as hierarchical trees, as the one represented in Fig. 2.1. A thesaurus includes other types of relations besides subsumption, for example specifying that caffeine has role mutagen.

\section{Gold Related Documents}

The importance of these relations can be easily understood by considering the domain modeled by the ontology in Fig. 2.1, and the need to find texts related to gold. Assume a corpus with one distinct document mentioning each metal, except for gold that no document mentions. So, which documents should we read first?

The document mentioning silver is probably the most related since it shares with gold two parents, precious and coinage. However, choos- 
Fig. 2.1 A DAG

representing a

classification of metals with multiple inheritance, since gold and silver are considered both precious and coinage metals (All the links represent $i s-a$ relations)

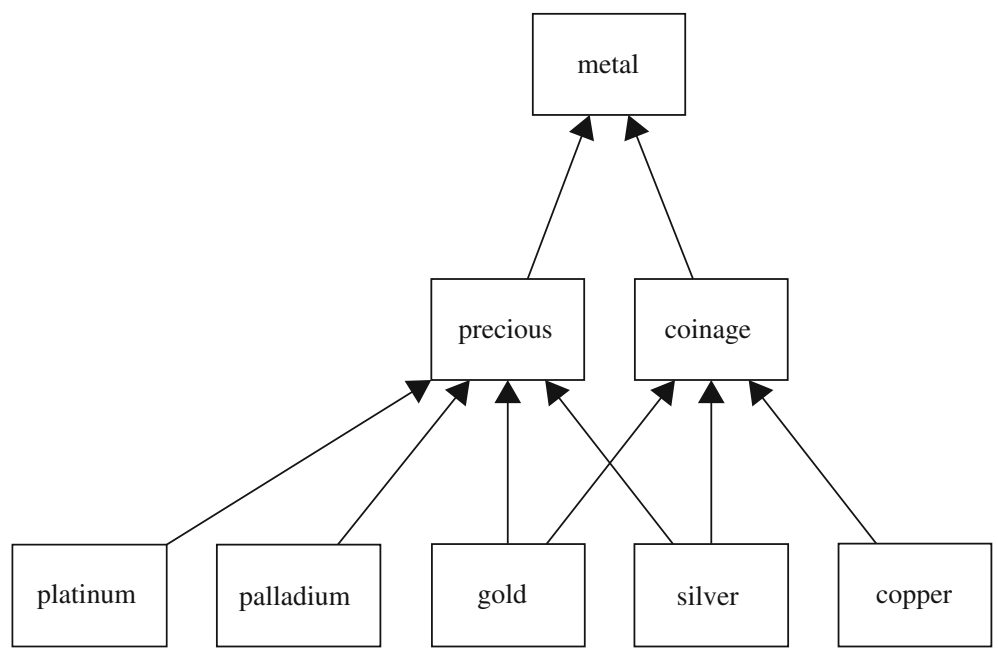

ing between the documents mentioning platinum or palladium or the document mentioning copper depends on our information need. This information can be obtained by our previous searches or reads. For example, assuming that our last searches included the word coinage, then document mentioning copper is probably the secondmost related. The importance of these semantic resources is evidenced by the development of the knowledge graph ${ }^{24}$ by Google to enhance their search engine (Singhal 2012).

\section{Where?}

Most of the biomedical ontologies are available through BioPortal ${ }^{25}$. In December of 2018, BioPortal provided access to more than 750 ontologies representing more than 9 million classes. BioPortal allows us to search for an ontology or a specific class. For example, if we search for caffeine, we will be able to see the large list of ontologies that define it. Each of these classes represent conceptualizations of caffeine in different domains and using alternative perspectives. To improve interoperability some ontologies include class properties with a link to similar classes in other ontologies. One of the main goals of the OBO initiative was precisely to tackle this somehow disorderly spread of definitions for the same concepts. Each OBO ontology covers a clearly specified scope that is clearly identified.

\section{OBO Ontologies}

A major example of success of $\mathrm{OBO}$ ontologies is the Gene Ontology (GO) that has been widely and consistently used to describe the molecular function, biological process and cellular component of gene-products, in a uniform way across different species (Ashburner et al. 2000). Another OBO ontology is the Disease Ontology (DO) that provides human disease terms, phenotype characteristics and related medical vocabulary disease concepts (Schriml et al. 2018). Another OBO ontology is the Chemical Entities of Biological Interest (ChEBI) that provides a classification of molecular entities with biological interest with a focus on small chemical compounds (Degtyarenko et al. 2007).

\section{Popular Controlled Vocabularies}

Besides OBO ontologies, other popular controlled vocabularies also exist. One of them is the International Classification of Diseases (ICD) ${ }^{26}$, maintained by the World Health Organization (WHO). This vocabulary contains a list of

\footnotetext{
${ }^{26}$ https://www.who.int/classifications/icd/en/
}

\footnotetext{
${ }^{24}$ https://en.wikipedia.org/wiki/Knowledge_Graph

${ }^{25} \mathrm{http} / / /$ bioportal.bioontology.org/
} 
generic clinical terms mainly arranged and classified according to anatomy or etiology. Another example is the Systematized Nomenclature of Medicine - Clinical Terms (SNOMED CT) ${ }^{27}$, currently maintained and distributed by the International Health Terminology Standards Development Organization (IHTSDO). The SNOMED CT is a highly comprehensive and detailed set of clinical terms used in many biomedical systems. The Medical Subject Headings $(\mathrm{MeSH})^{28}$ is a comprehensive controlled vocabulary maintained by the National Library of Medicine (NLM) for classifying biomedical and health-related information and documents. Both MeSH and SNOMED CT are included in the Metathesaurus of the Unified Medical Language System (UMLS) ${ }^{29}$, maintained by the U.S National Library of Medicine. This is a large resource that integrates most of the available biomedical vocabularies. The 2015AB release covered more than three million concepts.

Another alternative to BioPortal is Ontobee ${ }^{30}$, a repository of ontologies used by most OBO ontologies, but it also includes many non-OBO ontologies. In December 2018, Ontobee provided access to 187 ontologies (Ong et al. 2016).

Other alternatives outside the biomedical domain include the list of vocabularies gathered by the W3C SWEO Linking Open Data community project $^{31}$, and by the W3C Library Linked Data Incubator Group ${ }^{32}$.

\section{How?}

After finding the ontologies that cover our domain of interest in the previous catalogs, a good idea is to find their home page and download the

\footnotetext{
${ }^{27}$ https://digital.nhs.uk/services/terminology-andclassifications/snomed-ct

${ }^{28}$ https://www.nlm.nih.gov/mesh/

${ }^{29} \mathrm{https} / / / \mathrm{www} . n l m . n i h . g o v /$ research/umls/

${ }^{30} \mathrm{http}: / / \mathrm{www}$.ontobee.org/

${ }^{31} \mathrm{http} / / / w w w . w 3 . o r g / w i k i / T a s k F o r c e s /$

CommunityProjects/LinkingOpenData/

CommonVocabularies

${ }^{32} \mathrm{http} / / / w w w . w 3 . o r g / 2005 /$ Incubator/lld/XGR-1ldvocabdataset-20111025
}

files from there. This way, we will be sure that we get the most recent release in the original format and select the subset of the ontology that really matter for our work. For example, ChEBI provides three versions: LITE, CORE and FULL $^{33}$. Since we are interested in using the ontology just for text processing, we are probably not interested in chemical data and structures that is available in CORE. Thus, LITE is probably the best solution, and it will be the one we will use in this book. However, we may be missing synonyms that are only included in the FULL version.

\section{OWL}

The OWL language is the prevailing language to represent ontologies, and for that reason will be the format we will use in this book. OWL extends RDF Schema (RDFS) with more complex statements using description logic. RDFS is an extension of RDF with additional statements, such as class-subclass or property-subproperty relationships. RDF is a data model that stores information in statements represented as triples of the form subject, predicate and object. Originally, W3C recommended RDF data to be encoded using Extensible Markup Language (XML) syntax, also named RDF/XML. XML is a selfdescriptive mark-up language composed of data elements.

For example, the following example represents an XML file specifying that caffeine is a drug that may treat the condition of sleepiness, but without being an official treatment:

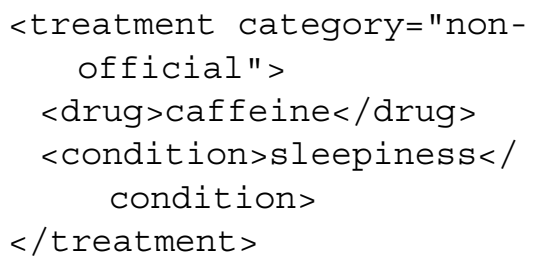

The information is organized in an hierarchical structure of data elements. treatment is the parent element of drug and condition. The character $<$ means that a new data element is being specified, and the characters $</$ means

\footnotetext{
${ }^{33}$ https://www.ebi.ac.uk/chebi/downloadsForward.do
} 
that a specification of data element will end. The treatment element has a property named category with the value non-official. The drug and condition elements have as values caffeine and sleepiness, respectively. This is a very simple XML example, but large XML files are almost unreadable by humans.

To address this issue other encoding languages for RDF are now being used, such as $N 3^{34}$ and Turtle $^{35}$. Nevertheless, most biomedical ontologies are available in OWL using XML encoding.

\section{URI}

The Uniform Resource Identifier (URI) was defined as the standard global identifier of classes in an ontology. For example, the class caffeine in ChEBI is identified by the following URI:

\section{http://purl.obolibrary.org/obo/ CHEBI 27732}

If a URI represents a link to a retrievable resource is considered a Uniform Resource Locator, or URL. In other words, a URI is a URL if we open it in a web browser and obtain a resource describing that class.
Sometimes, ontologies are also available as database dumps. These dumps are normally SQL files that need to be fed to a DataBase Management System (DBMS) ${ }^{36}$. If for any reason we must deal with these files, we can use the simple command line tool named sqlite3. The tool has the option to execute the SQL commands to import the data into a database (.read command), and to export the data into a CSV file (. mode command) (Allen and Owens 2011).

\section{Further Reading}

One important read if we need to know more about biomedical resources is the Arthur Lesk's book about bioinformatics (Lesk 2014). The book has entire chapters dedicated to where data and text can be found, providing a comprehensive overview of the type of biomedical information available, nowadays.

A more pragmatic approach is to explore the vast number of manuals, tutorials, seminars and courses provided by the $\mathrm{EBI}^{37}$ and $\mathrm{NCBI}^{38}$.

\footnotetext{
${ }^{36}$ https://en.wikipedia.org/wiki/Database\#

Database_management_system

${ }^{37}$ https://www.ebi.ac.uk/training

${ }^{38}$ https://www.ncbi.nlm.nih.gov/home/learn/
}

\footnotetext{
${ }^{34}$ https://en.wikipedia.org/wiki/Notation3

${ }^{35}$ https://en.wikipedia.org/wiki/Turtle_(syntax)
}

Open Access This chapter is licensed under the terms of the Creative Commons Attribution 4.0 International License (http://creativecommons.org/licenses/by/4.0/), which permits use, sharing, adaptation, distribution and reproduction in any medium or format, as long as you give appropriate credit to the original author(s) and the source, provide a link to the Creative Commons licence and indicate if changes were made.

The images or other third party material in this chapter are included in the chapter's Creative Commons licence, unless indicated otherwise in a credit line to the material. If material is not included in the chapter's Creative Commons licence and your intended use is not permitted by statutory regulation or exceeds the permitted use, you will need to obtain permission directly from the copyright holder. 


\section{Data Retrieval}

\begin{abstract}
This chapter starts by introducing an example of how we can retrieve text, where every step is done manually. The chapter will describe step-by-step how we can automatize each step of the example using shell script commands, which will be introduced and explained as long as they are required. The goal is to equip the reader with a basic set of skills to retrieve data from any online database and follow the links to retrieve more information from other sources, such as literature.
\end{abstract}

\section{Keywords}

Unix shell · Terminal application - Web retrieval · cURL: Client Uniform Resource Locator - Data extraction - Data selection . Data filtering $\cdot$ Pattern matching $\cdot$ XML: extensible markup language $\cdot \mathrm{XPath}: \mathrm{XML}$ path language

\section{Caffeine Example}

As our main example, let us consider that we need to retrieve more data and literature about caffeine. If we really do not know anything about caffeine, we may start by opening our favorite internet browser and then searching caffeine in Wikipedia ${ }^{1}$ to know what it really

\footnotetext{
${ }^{1}$ https://en.wikipedia.org/wiki/Caffeine
}

is (see Fig. 3.1). From all the information that is available we can check in the infobox that there are multiple links to external sources. The infobox is normally a table added to the top right-hand part of a web page with structured data about the entity described on that page.

From the list of identifiers (see Fig. 3.2), let us select the link to one resource hosted by the European Bioinfomatics Institute (EBI), the link to CHEBI:27732².

CHEBI represents the acronym of the resource Chemical Entities of Biological Interest $(\mathrm{ChEBI})^{3}$ and 27732 the identifier of the entry in ChEBI describing caffeine (see Fig. 3.3). ChEBI is a freely available database of molecular entities with a focus on "small" chemical compounds. More than a simple database, ChEBI also includes an ontology that classifies the entities according to their structural and biological properties.

By analyzing the CHEBI:27732 web page we can check that ChEBI provides a comprehensive set of information about this chemical compound. But let us focus on the Automatic Xrefs tab ${ }^{4}$. This tab provides a set of external links to other

\footnotetext{
${ }^{2}$ https://www.ebi.ac.uk/chebi/searchId.do?chebiId= CHEBI:27732

${ }^{3}$ http://www.ebi.ac.uk/chebi/

${ }^{4}$ http://www.ebi.ac.uk/chebi/displayAutoXrefs.do? chebiId=CHEBI: 27732
} 


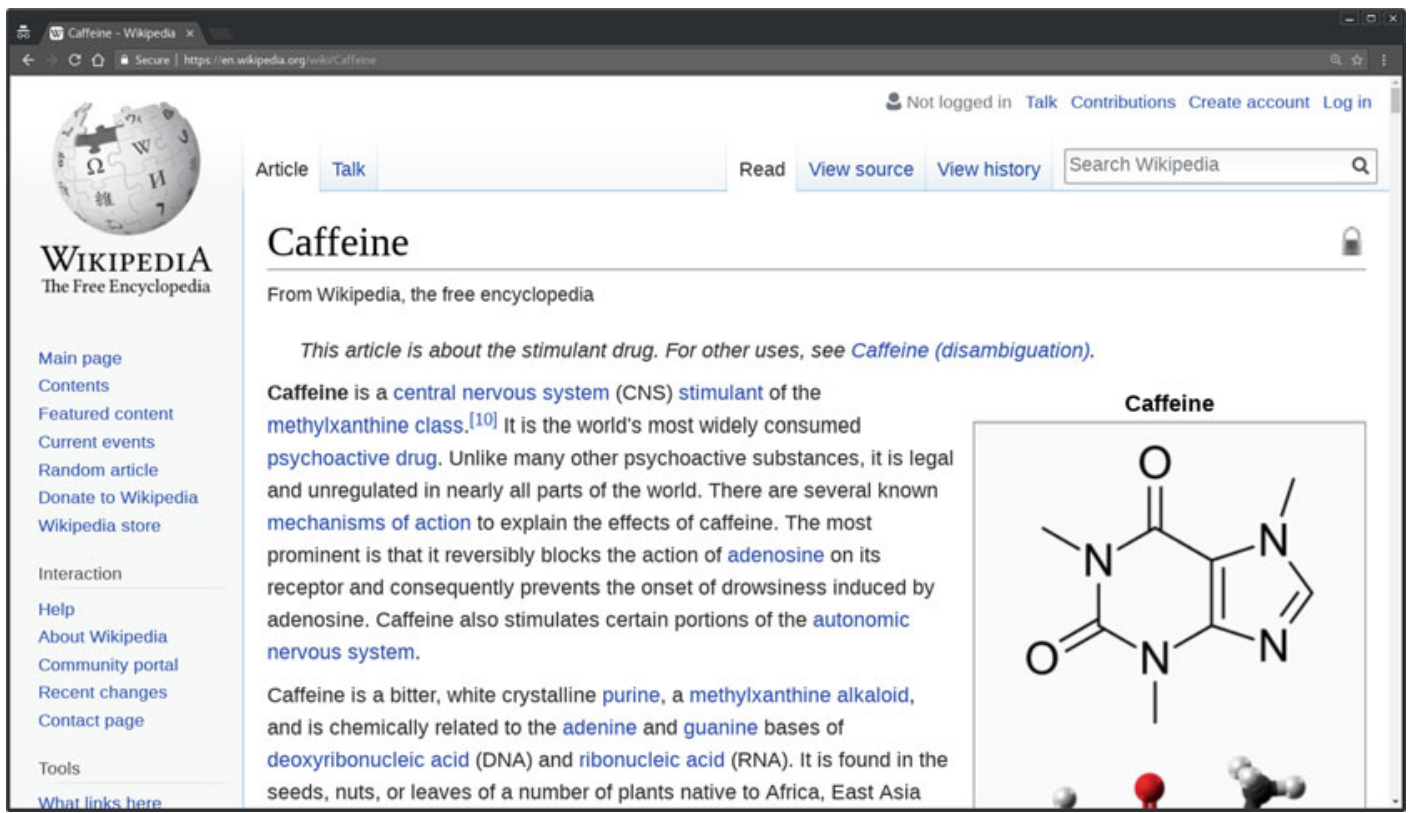

Fig. 3.1 Wikipedia page about caffeine

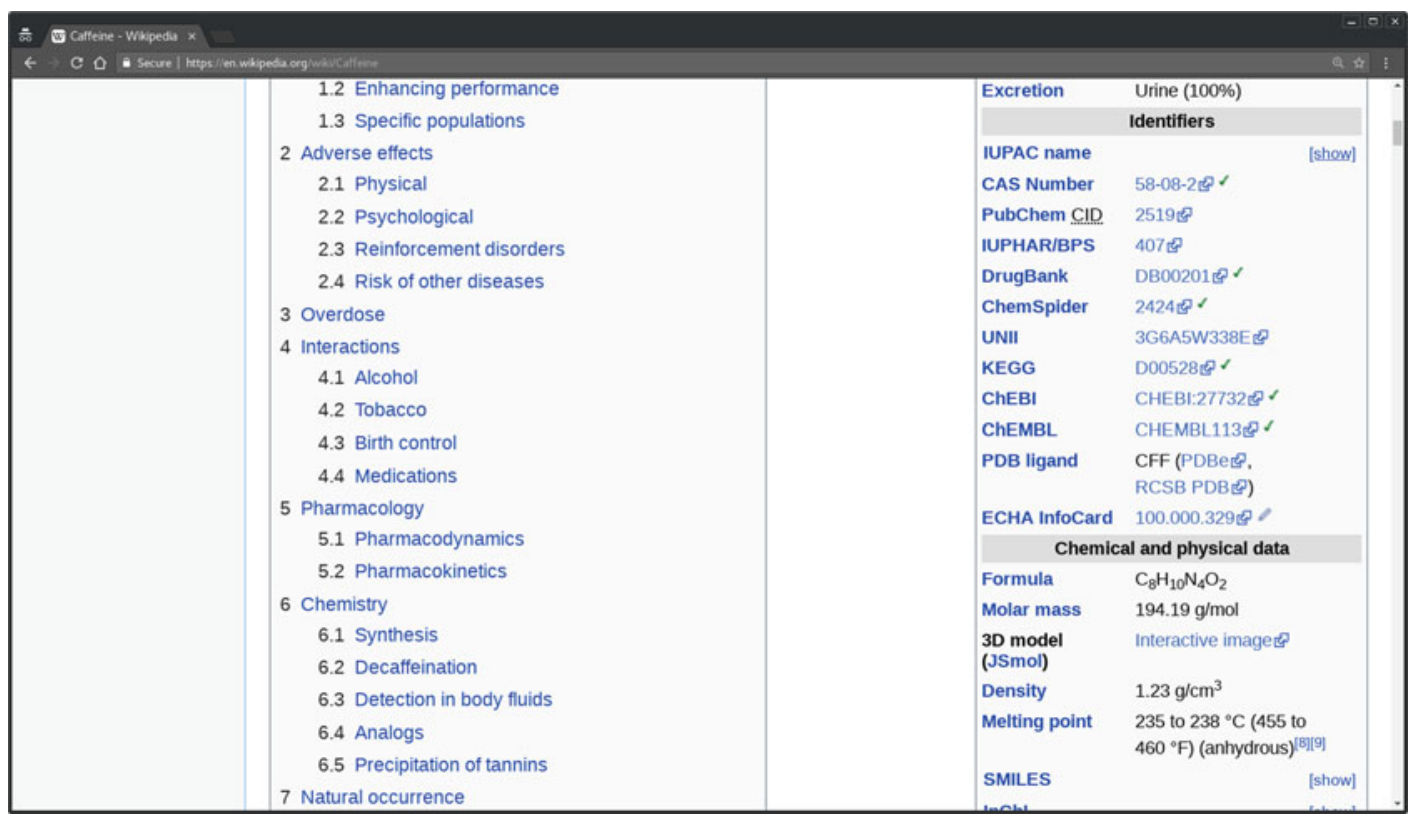

Fig. 3.2 Identifiers section of the Wikipedia page about caffeine

resources describing entities somehow related to the complete list ${ }^{5}$ (see Fig. 3.5). These links caffeine (see Fig. 3.4).

In the Protein Sequences section, we have are to another resource hosted by the EBI, the 77 proteins (in September of 2018) related to caffeine. If we click on show all we will get 


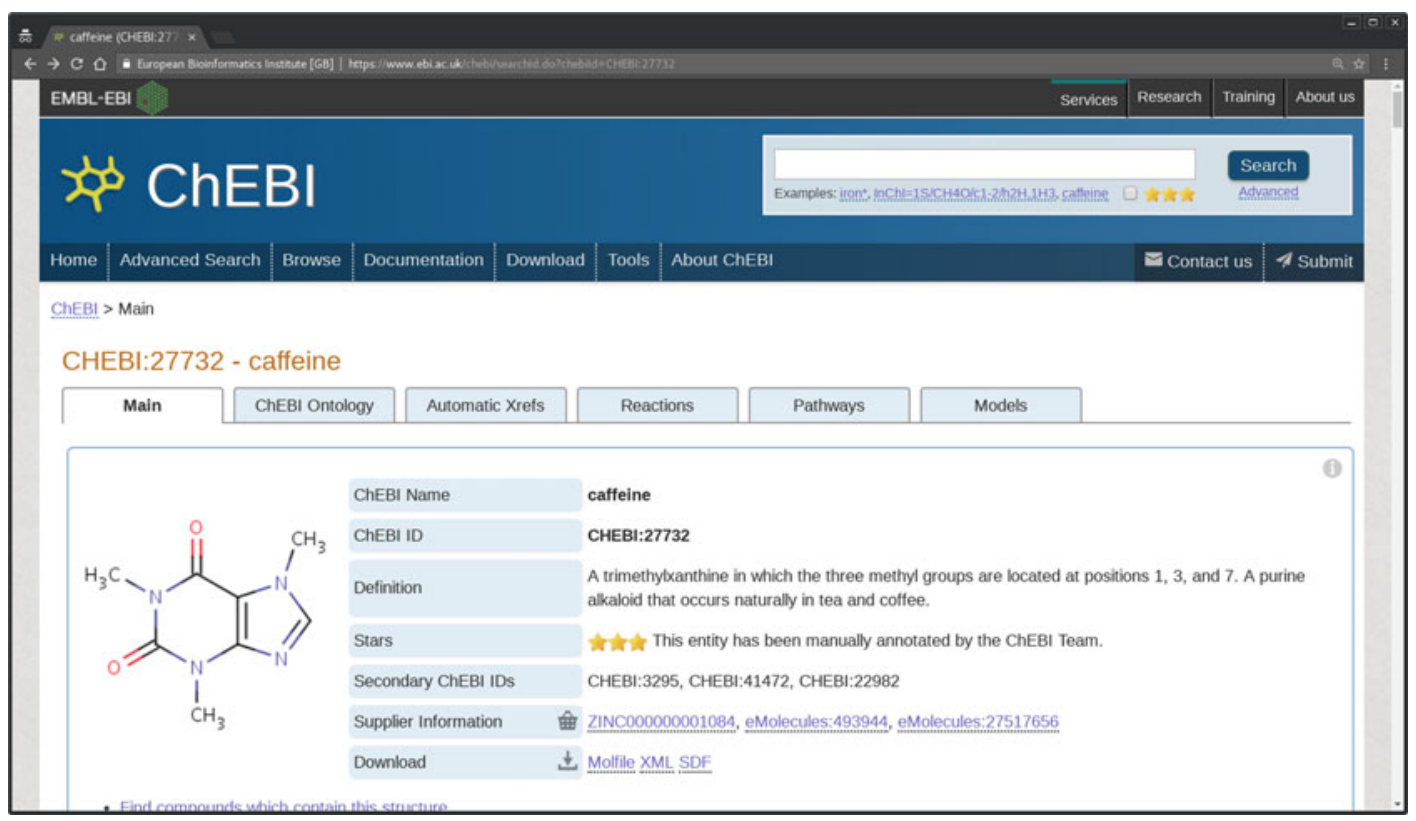

Fig. 3.3 ChEBI entry describing caffeine

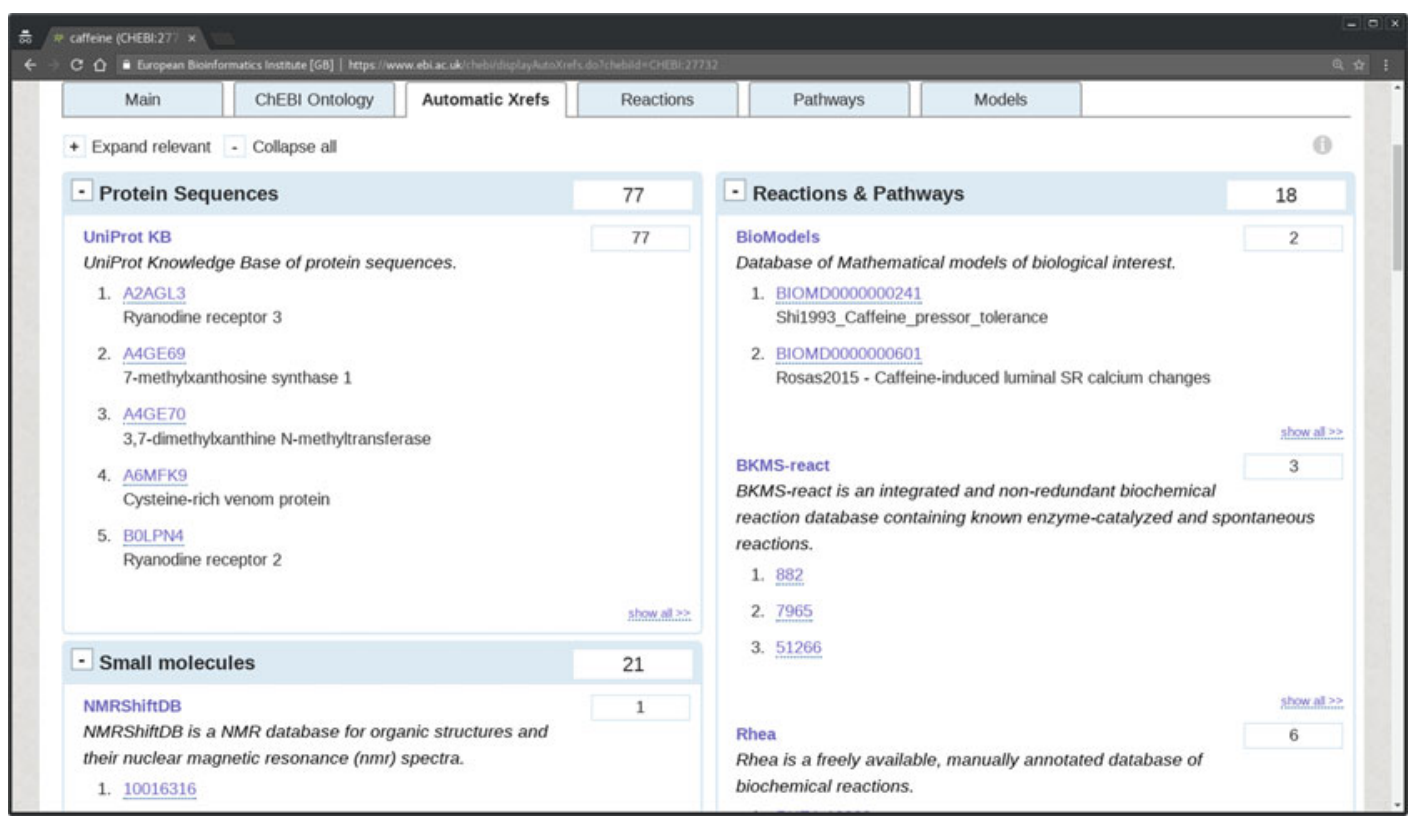

Fig. 3.4 External references related to caffeine

UniProt, a database of protein sequences and annotation data.

The list includes the identifiers of each protein with a direct link to respective entry in UniProt, the name of the protein and some topics about the description of the protein. For example,
DISRUPTION PHENOTYPE means some effects caused by the disruption of the gene coding for the protein are known ${ }^{6}$.

${ }^{6}$ https://web.expasy.org/docs/userman.html\#CC_line 


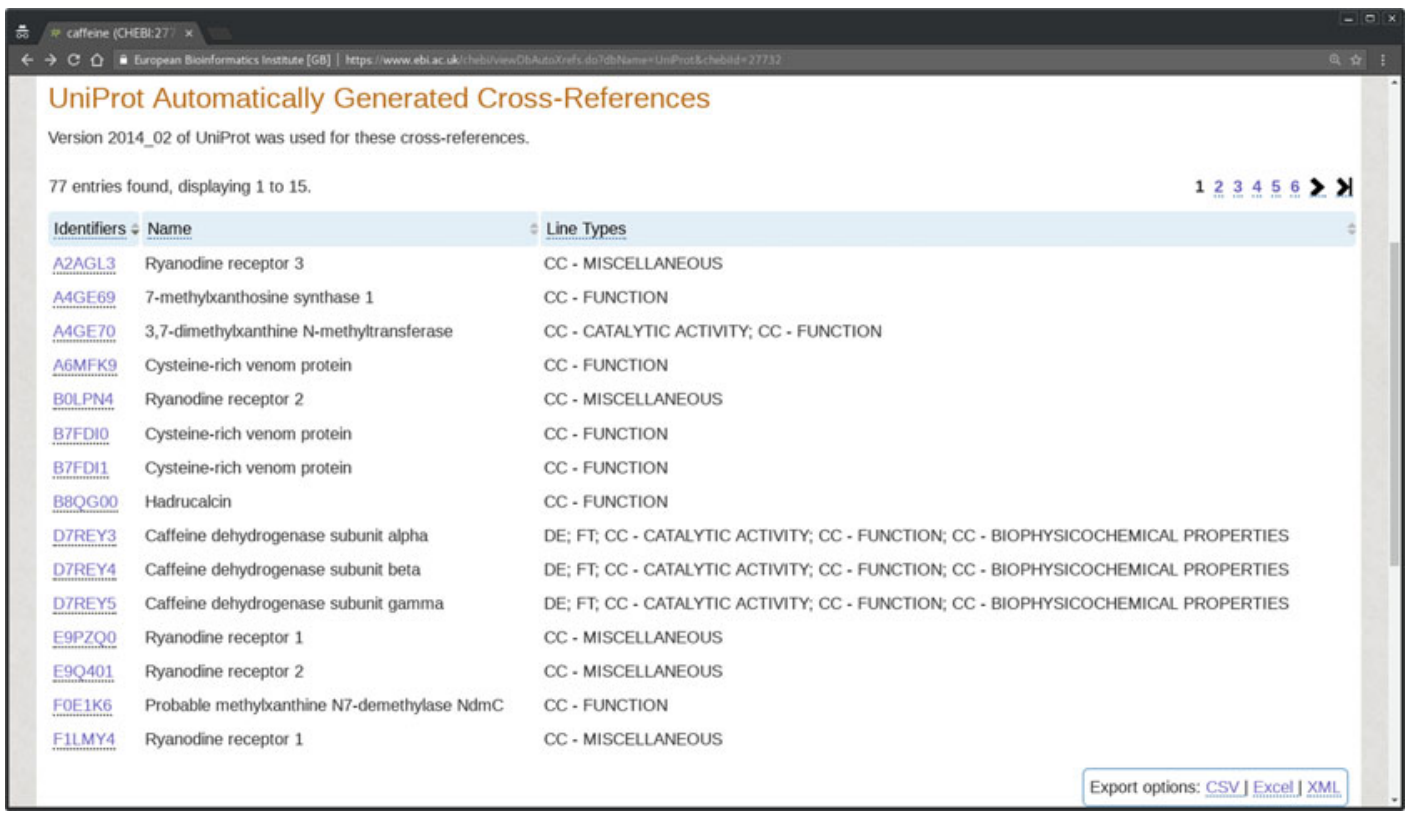

Fig. 3.5 Proteins related to caffeine

We should note that at bottom-right of the page there are Export options that enable us to download the full list of protein references in a single file. These options include:

CSV: Comma Separated Values, the open format file that enable us to store data as a single table format (columns and rows).

Excel: a proprietary format designed to store and access the data using the software Microsoft Excel.

XML: eXtensible Markup Language, the open format file that enable us to store data using a hierarchy of markup tags.

We start by downloading the CSV, Excel and XML files. We can now open the files and check its contents in a regular text editor software ${ }^{7}$ installed in our computer, such as notepad (Windows), TextEdit (Mac) or gedit (Linux).

The first lines of the chebi_27732_xrefs_ UniProt.csv file should look like this:

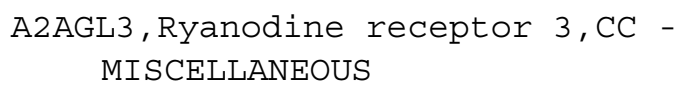

${ }^{7}$ https://en.wikipedia.org/wiki/Text_editor
A4GE69, 7-methylxanthosine synthase 1,CC - FUNCTION

The first lines of the chebi_27732_xrefs_ UniProt.xls file should look like this:

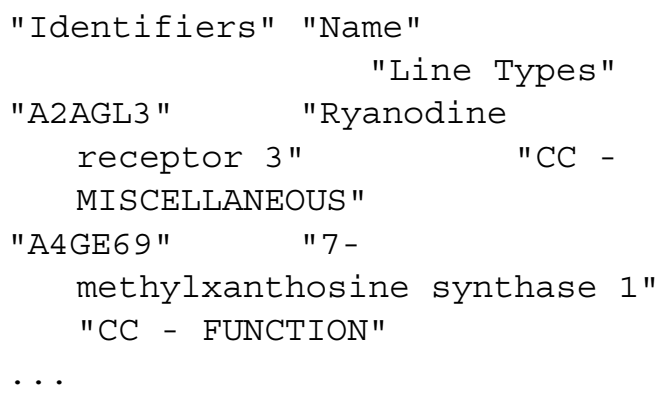

As we can see, this is not the proprietary format XLS but instead a TSV format. Thus, the file can still be open directly on Microsoft Excel.

The first lines of the chebi_27732_xrefs_ UniProt.xml file should look like this:

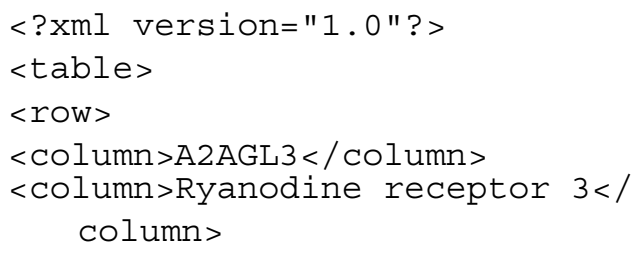




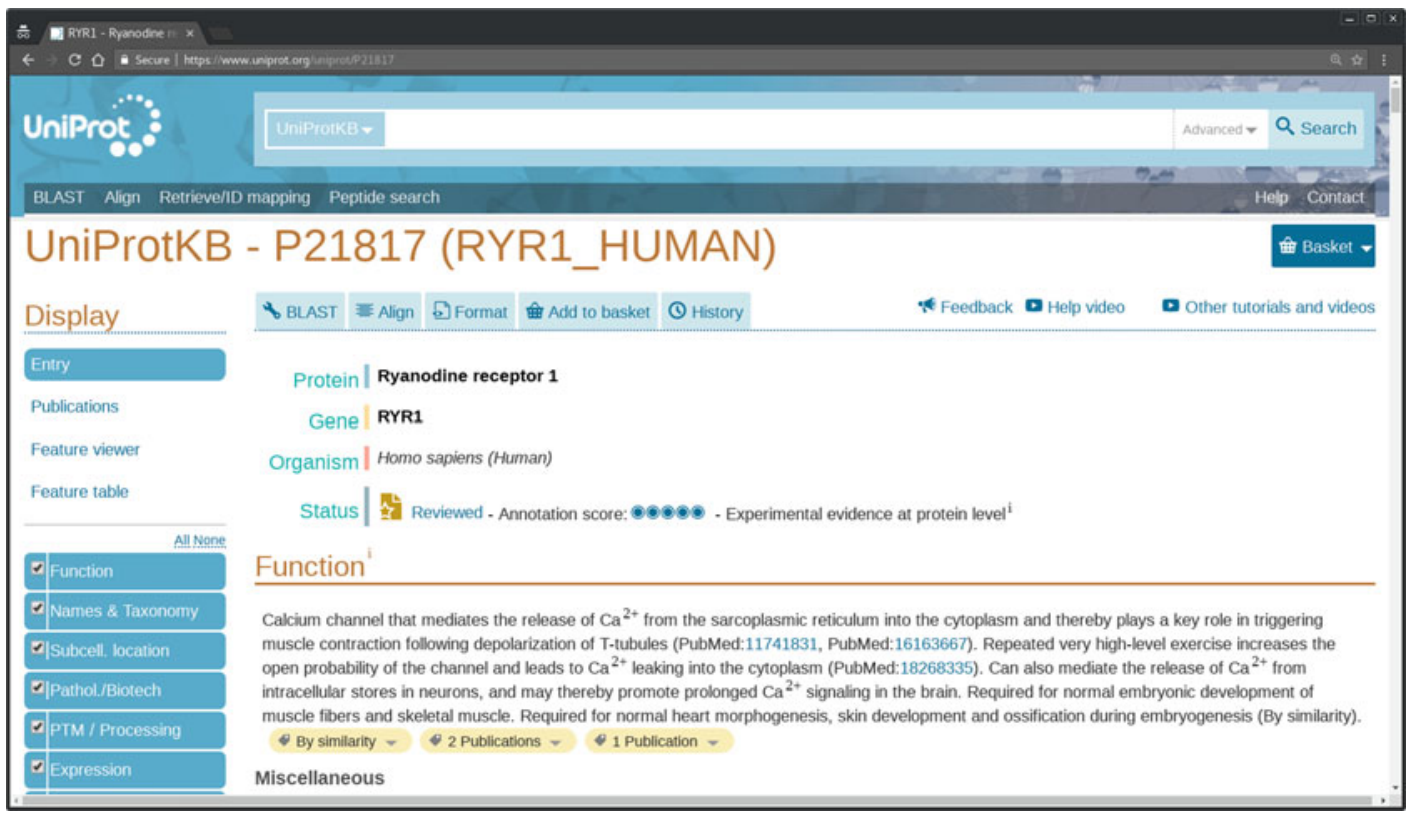

Fig. 3.6 UniProt entry describing the Ryanodine receptor 1

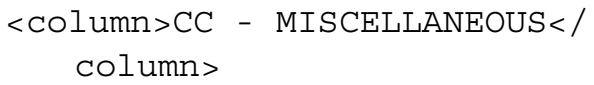

$</$ row $>$

$<$ row $>$

$<$ column >A4GE69</ Column>

$<$ column> 7-methylxanthosine

synthase $1</$ column $>$

$<$ COlumn $>$ CC - FUNCTION $</$ COlumn $>$

$</$ row $>$

...

We should note that all the files contain the same data they only use a different format.

If for any reason, we are not able to download the previous files from UniProt, we can get them from the book file archive ${ }^{8}$.

In the following sections we will use these files to automatize this process, but for now let us continue our manual exercise using the internet browser. Let us select the Ryanodine receptor 1 with the identifier P21817 and click on the link ${ }^{9}$ (see Fig. 3.6). We can now see that UniProt is

\footnotetext{
${ }^{8}$ http://labs.rd.ciencias.ulisboa.pt/book/

${ }^{9}$ http://www.uniprot.org/uniprot/P21817
}

much more than just a sequence database. The sequence is just a tiny fraction of all the information describing the protein. All this information can also be downloaded as a single file by clicking on Format and on XML. Then, save the result as a XML file to our computer.

Again, we can use our text editor to open the downloaded file named P21817.xml, which first lines should look like this:

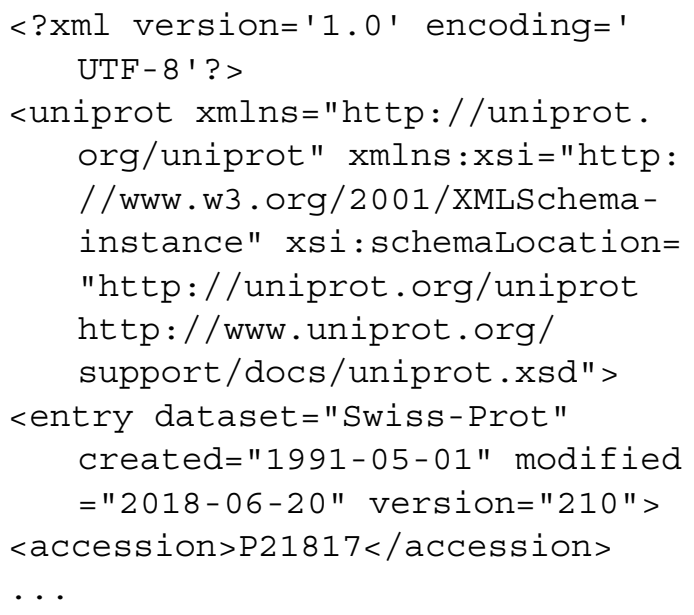

We can check that this entry represents a Homo sapiens (Human) protein, so if we are interested only in Human Proteins, we will have 


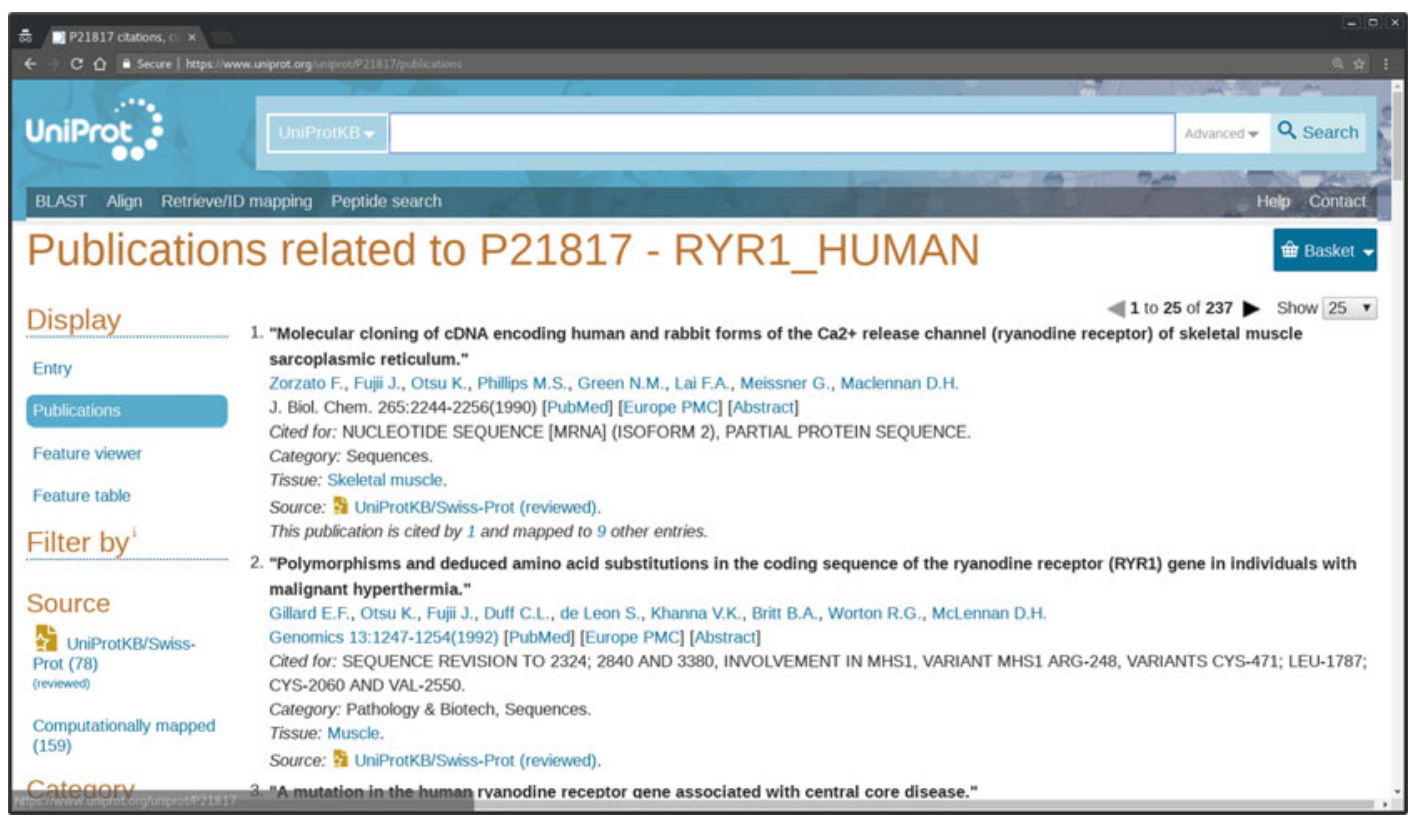

Fig. 3.7 Publications related to Ryanodine receptor 1

to filter them. For example, the entry E9PZQ0 ${ }^{10}$ in the ChEBI list also represents a Ryanodine receptor 1 protein but for the Mus musculus (Mouse).

Going back to the browser in the top-left side of the UniProt entry we have a link to publications ${ }^{11}$. If we click on it, we will see a list of publications somehow related to the protein (see Fig. 3.7).

Let us assume that we are interested in finding phenotypic information, the first title that may attract our attention is: Polymorphisms and deduced amino acid substitutions in the coding sequence of the ryanodine receptor (RYRI) gene in individuals with malignant hyperthermia. To know more about the publication, we can use the UniProt citations service by clicking on the Abstract link $^{12}$ (see Fig. 3.8).

To check if the abstract mentions any disease we can use an online text mining tool, for example the Minimal Named-Entity Recognizer $(\mathrm{MER})^{13}$. We can copy and paste the abstract of

\footnotetext{
${ }^{10} \mathrm{http}$ ///www.uniprot.org/uniprot/E9PZQ0

${ }^{11} \mathrm{https}$ ///www.uniprot.org/uniprot/P21817/publications

${ }^{12} \mathrm{https}$ ///www.uniprot.org/citations/1354642

${ }^{13}$ http://labs.rd.ciencias.ulisboa.pt/mer/
}

the publication into MER and select $\mathrm{DO}-\mathrm{Hu}$ man Disease Ontology as lexicon (see Fig. 3.9).

We will see that MER detects three mentions of malignant hyperthermia, giving us another link ${ }^{14}$ about the disease found (see Fig. 3.10).

Thus, in summary, we started from a generic definition of caffeine and ended with an abstract about hyperthermia by following the links in different databases. Of course, this does not mean that by taking caffeine we will get hyperthermia, or that we will treat hyperthermia by taking caffeine (maybe as a cold drink $\ddot{\cup}^{15}$ ). However, this relation has a context, a protein and a publication, that need to be further analyzed before drawing any conclusions.

We should note that we only analyzed one protein and one publication, we now need to repeat all the steps to all the proteins and to all the publications related to each protein. And this could even be more complicated if we were interested in other central nervous system stimulants, for example by looking in the ChEBI

\footnotetext{
${ }^{14}$ http://purl.obolibrary.org/obo/DOID_8545

${ }^{15}$ https://en.wikipedia.org/wiki/Hyperthermia\#Treatment
} 


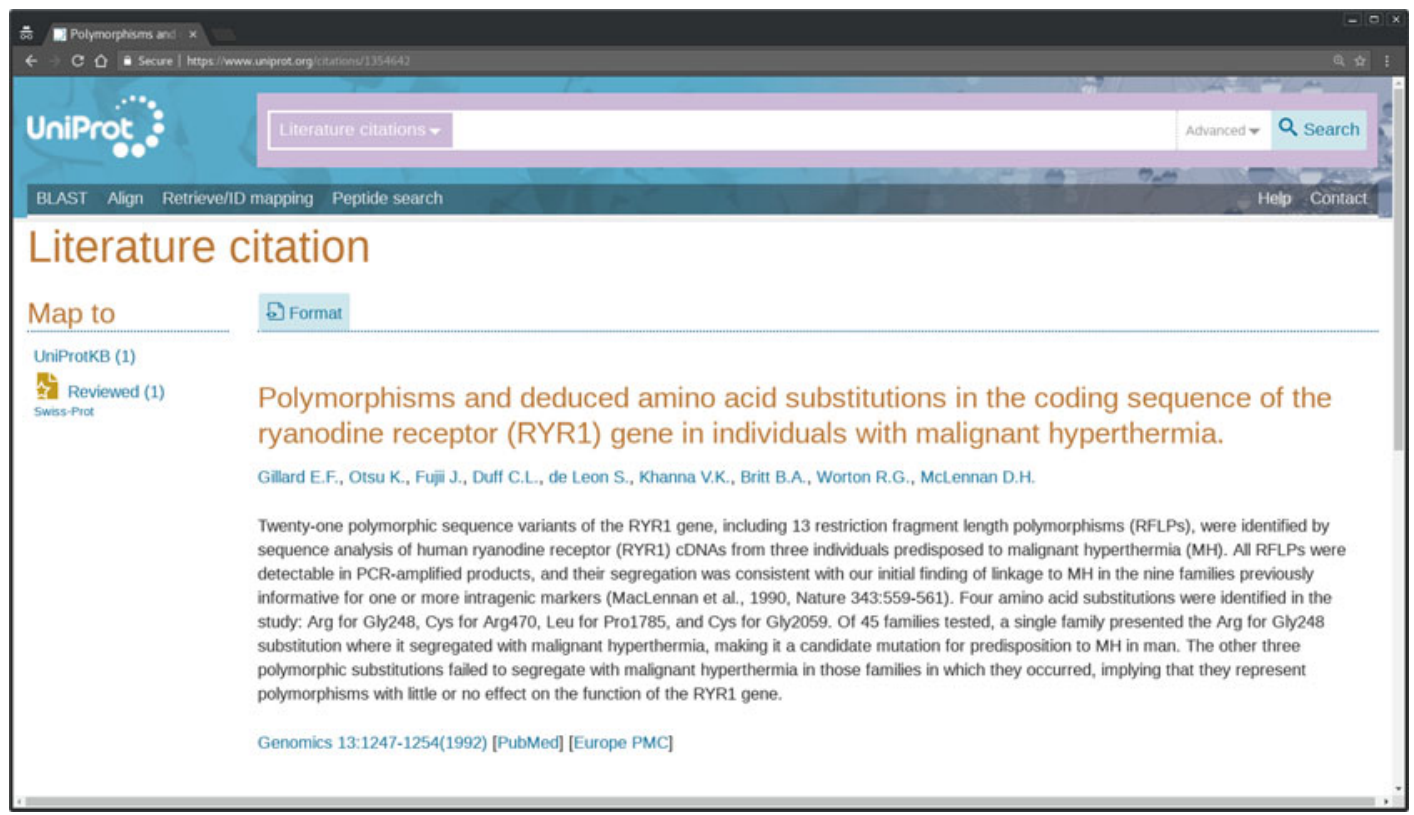

Fig. 3.8 Abstract of the publication entitled Polymorphisms and deduced amino acid substitutions in the coding sequence of the ryanodine receptor (RYR1) gene in individuals with malignant hyperthermia

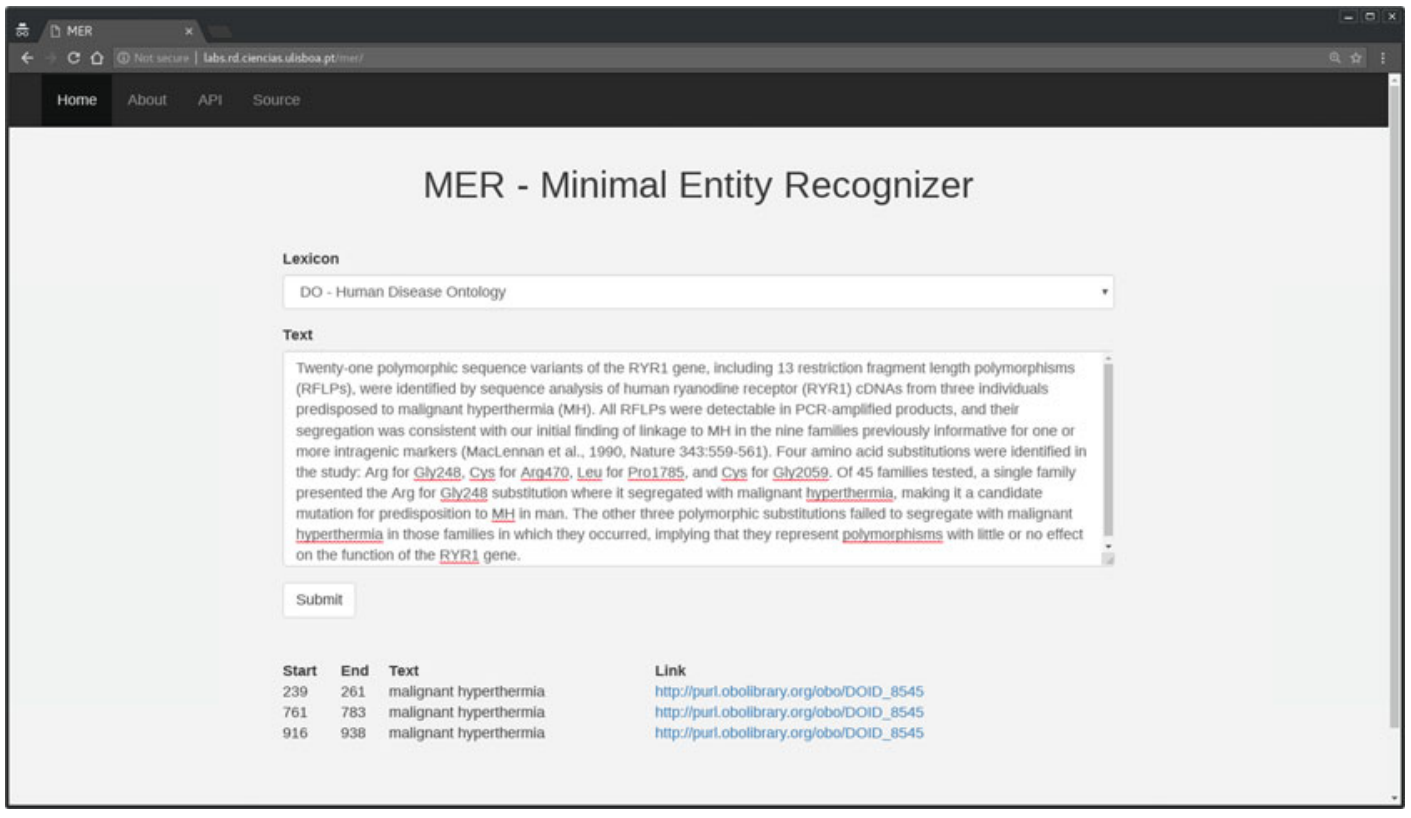

Fig. 3.9 Diseases recognized by the online tool MER in an abstract

ontology ${ }^{16}$. This is of course the motivation to automatize the process, since it is not humanly

${ }^{16}$ https://www.ebi.ac.uk/chebi/chebiOntology.do? chebiId $=35337$ feasible to deal with such large amount of data, that keeps evolving every day.

However, if the goal was to find a relation between caffeine and hyperthermia, we could simply have searched these two terms in PubMed. 


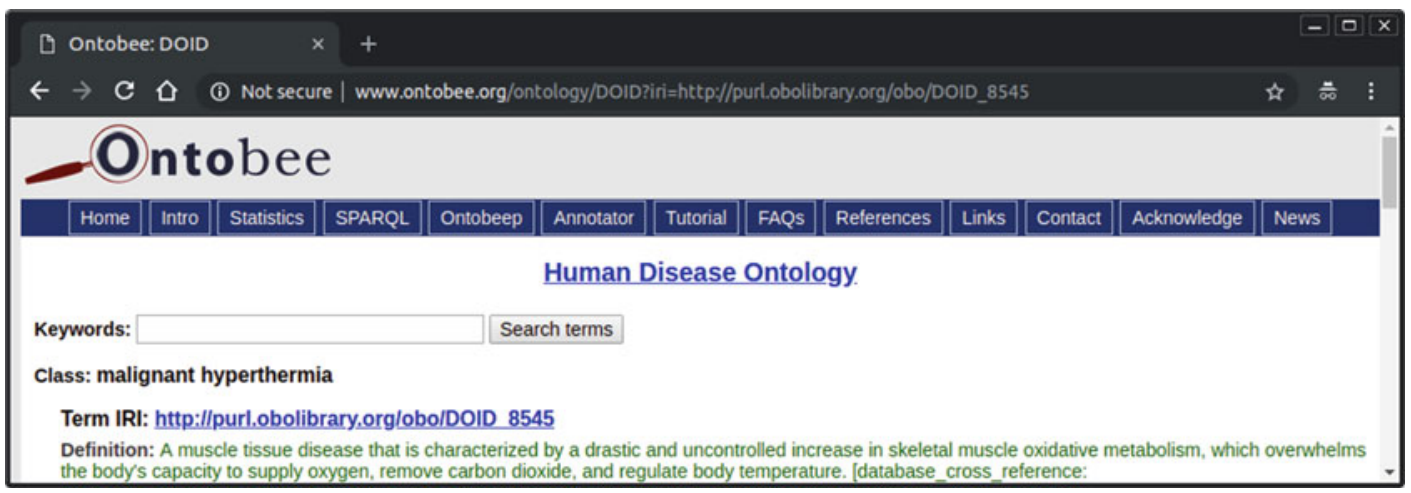

Fig. 3.10 Ontobee entry for the class malignant hyperthermia

We did not do that because some relations are not explicitly mention in the text, thus we have to navigate through database links. The second reason is because we needed an example using different resources and multiple entries to explain how we can automate most of these steps using shell scripting. The automation of the example will introduce a comprehensive set of techniques and commands, which with some adaptation Life and Health specialists can use to address many of their text and data processing challenges.

\section{Unix Shell}

The first step is to open a shell in our personal computer. A shell is a software program that interprets and executes command lines given by the user in consecutive lines of text. A shell script is a list of such command lines. The command line usually starts by invoking a command line tool. This manuscript will introduce a few command line tools, which will allow us to automatize the previous example. Unix shell was developed to manage Unix-like operating systems, but due to their usefulness nowadays they are available is most personal computers using Linux, macOS or Windows operating systems. There are many types of Unix shells with minor differences between them (e.g. sh, ksh, csh, tcsh and bash), but the most widely available is the Bourne-Again shell $\left(\right.$ bash $^{17}$ ). The examples in this manuscript were tested using bash.

${ }^{17}$ https://en.wikipedia.org/wiki/Bash_(Unix_shell)
So, the first step is to open a shell in our personal computer using a terminal application (see Fig. 3.11). If we are using Linux or macOS then this is usually not new for us, since most probably we have a terminal application already installed, that opens a shell for us. In case we are using a Microsoft Windows operating system, then we have several options to consider. If we are using Windows 10, then we can install a Windows Subsystem for Linux ${ }^{18}$ or just install a third-party application, such as MobaXterm ${ }^{19}$. No matter which terminal application we end up using, the shell will always have a common look: a text window with a cursor blinking waiting for our first command line. We should note that most terminal applications allow the usage of the up and down cursor keys to select, edit, and execute previous commands, and the usage of the tab key to complete the name of a command or a file.

\section{Current Directory}

As our first command line, we can type: pwd

After hitting enter, the command will show the full path of the directory (folder) of our computer in which the shell is working on. The dollar sign in the left is only to indicate that this is a command to be executed directly in the shell.

\footnotetext{
${ }^{18} \mathrm{https} / / /$ docs.microsoft.com/en-us/windows/wsl/about

${ }^{19}$ https://mobaxterm.mobatek.net/
} 


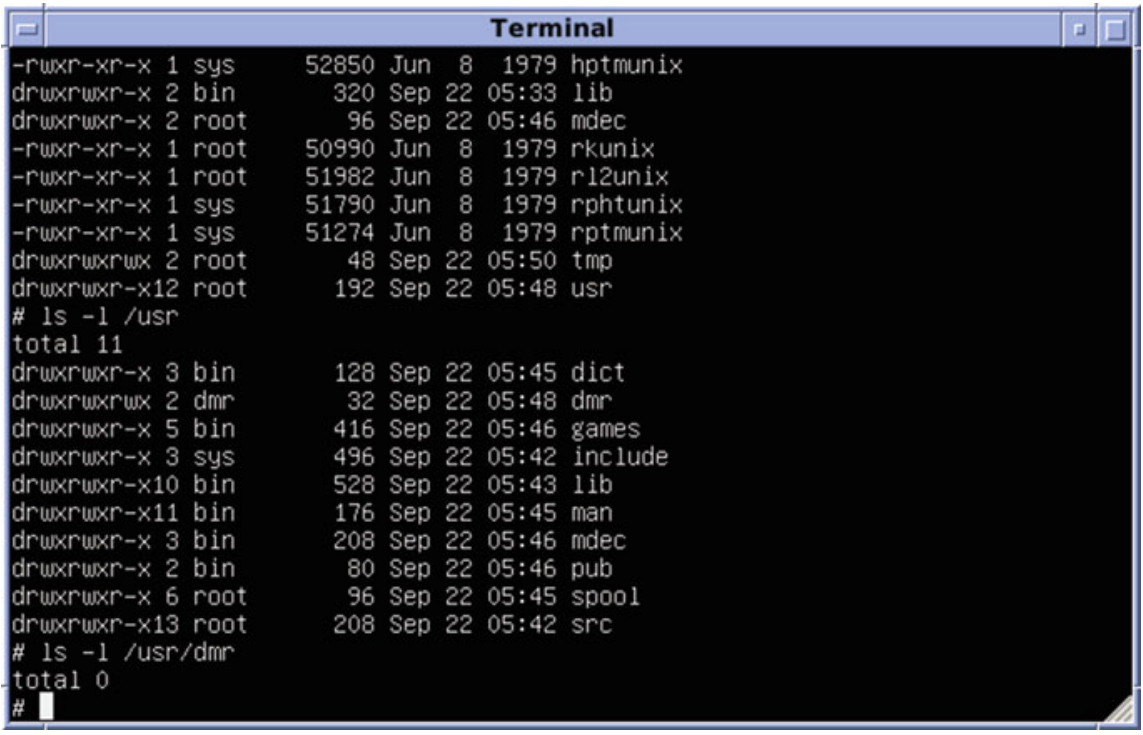

Fig. 3.11 Screenshot of a Terminal application (Source: https://en.wikipedia.org/wiki/Unix)

To understand a command line tool, such as pwd, we can type man followed by the name of the tool. For example, we can type man pwd to learn more about pwd (do not forget to hit enter, and press $q$ to quit). We can also learn more about man by typing man man. A shorter alternative to man, is to add the --help option after any command tool. For example, we can type pwd - - help to have a more concise description of pwd.

As our second command line, we can type Is and hit enter. It will show the list of files in the current directory. For example, we can type is - - help to have a concise description of $1 \mathrm{~s}$. Since we will work with files, that we need to open with a text editor or a spreadsheet application $^{20}$, such as LibreOffice Calc or Microsoft Excel, we should select a current directory that we can easily open in our file explorer application. A good idea is to open our favorite file explorer application, select a directory, and then check its full path ${ }^{21}$.

\footnotetext{
${ }^{20}$ https://en.wikipedia.org/wiki/Spreadsheet

${ }^{21}$ https://en.wikipedia.org/wiki/Path_(computing)
}

\section{Windows Directories}

Notice that in Windows the full path to a directory each name is separated by a backslash $(\backslash)$ while in a Unix shell is a forward slash (/).

For example, a Windows path to the Documents folder may look like:

\section{$\mathrm{C}: \backslash$ Users $\backslash$ MyUserName $\backslash$ Documents}

If we are using the Windows Subsystem for Linux $^{22}$, the previous folder must be accessed using the path:

/mnt/c/Users/MyUserName/

\section{Documents}

If we are using MobaXterm ${ }^{23}$, the following path should be used instead:

/drives/c/Users/MyUserName /

Documents

\footnotetext{
${ }^{22}$ https://www.howtogeek.com/261383/how-to-accessyour-ubuntu-bash-files-in-windows-and-your-windowssystem-drive-in-bash/

${ }^{23}$ https://mobaxterm.mobatek.net/documentation.html
} 


\section{Change Directory}

To change the directory, we can use another command line tool, the cd (change directory) followed by the new path. In a Linux system we may want to use the Documents directory. If the Documents directory is inside our current directory (shown using $1 \mathrm{~s}$ ), we only need to type:

\section{\$ cd Documents}

Now we can type pwd to see what changed.

And if we want to return to the parent directory, we only need to use the two dots . . :

$\$ \mathrm{~cd}$. .

And if we want to return to the home directory, we only need to use the tilde character $(\sim)$ :

$\$ \mathrm{~cd} \sim$

Again, we should type pwd to double check if we are in the directory we really want.

In Windows we may need to use the full path, for example:

\section{cd /mnt/c/Users/MyUserName/ Documents}

We should note that we need to enclose the path within single (or double) quotes in case it contains spaces:

\section{\$ cd 1/mnt/c/Users/MyUserName/ Documents'}

Later on, we will know more about the difference between using single or double quotes. For now, we may assume that they are equivalent. To know more about cd, we can type cd --help.

\section{Useful Key Combinations}

Every time the terminal is blocked by any reason, we can press both the control and $\mathrm{C}$ key at the same time ${ }^{24}$. This usually cancels the current tool being executed. For example, try using the $c d$ command with only one single quote:

$\$ c d$ '

${ }^{24}$ https://en.wikipedia.org/wiki/Control
This will block the terminal, because it is still waiting for a second single quote that closes the argument. Now press control-C, and the command will be aborted.

Now we can type again the previous command, but instead of pressing control-C we may also press control- $\mathrm{D}^{25}$. The combination control$\mathrm{D}$ indicates the terminal that it is the end of input. So, in this case, the cd command will not be canceled, but instead it is executed without the second single quote and therefore a syntax error will be shown on our display.

Other useful key combinations are the controlL that when pressed cleans the terminal display, and the control-insert and shift-insert that when pressed copy and paste the selected text, respectively.

\section{Shell Version}

The following examples will probably work in any Unix shell, but if we want to be certain that we are using bash we can type the following command, and check if the output says bash.

ps $-p \quad \$ \$$

ps is a command line tool that shows information about active processes running in our computer. The $-\mathrm{p}$ option selects a given process, and in this case $\$ \$$ represents the process running in our terminal application. In most terminal applications bash is the default shell. If this is not our case, we may need to type bash, hit enter and now we are using bash.

Now that we know how to use a shell, we can start writing and running a very simple script that reverse the order of the lines in a text file.

\section{Data File}

We start by creating a file named myfile.txt using any text editor, and adding the following lines:

line 1

line 2

\footnotetext{
${ }^{25}$ https://en.wikipedia.org/wiki/End-of-

Transmission_character
} 
line 3

line 4

We cannot forget to save it in our working directory, and check if it has the proper filename extension.

\section{File Contents}

To check if the file is really on our working directory, we can type:

cat myfile.txt

The contents of the file should appear in our terminal. cat is a simple command line tool that receives a filename as argument and displays its contents on the screen. We can type man cat or cat --help to know more about this command line tool.

\section{Reverse File Contents}

An alternative to cat tool is the tac tool. To try it, we only need to type:

tac myfile.txt

The contents of the file should also appear in our terminal, but now in the reverse order. We can type man tac or tac --help to know more about this command line tool.

\section{My First Script}

Now we can create a script file named reversemyfile.sh by using the text editor, and add the following lines:

\section{1 tac $\$ 1$}

We cannot forget to save the file in our working directory. \$1 represents the first argument after the script filename when invoking it. Each script file presented in this manuscript will include the line numbers in the left. This will helps us not only to identify how many lines the script contains, but also to distinguish a script file from the commands to be executed directly in the shell.

\section{Line Breaks}

A Unix file represents a single line break by a line feed character, instead of two characters (carriage return and line feed) used by Windows ${ }^{26}$. So, if we are using a text editor in Windows, we must be careful to use one that lets us save it as Unix file, for example the open source Notepad $++{ }^{27}$.

In case we do not have such text editor, we can also remove the extra carriage return by using the command line tool $t r$, that replaces and deletes characters:

$$
\begin{gathered}
\text { tr }-d{ }^{\prime} \backslash r r^{\prime}<\text { reversemyfile.sh } \\
>\text { reversemyfilenew.sh }
\end{gathered}
$$

The $-d$ option of $t r$ is used to remove a given character from the input, in this case $t r$ will delete all carriage returns $(\backslash r)$. Many command line options can be used in short form using a single dash (-), or in a long form using two dashes (--). In this tool, using the --delete option is equivalent to the - $d$ option. Long forms are more self-explanatory, but they take longer to type and occupy more space. We can type man tr or tr - -help to know more about this command line tool.

\section{Redirection Operator}

The $>$ character represents a redirection operator $^{28}$ that moves the results being displayed at the standard output (our terminal) to a given file. The $<$ character represents a redirection operator that works on the opposite direction, i.e. opens a given file and uses it as the standard input.

We should note that cat received the filename as an input argument, while tr can only receive the contents of the file through the standard input. Instead of providing the filename as argument, the cat command can also receive the contents of a file through the standard input, and produce the same output:

\footnotetext{
${ }^{26}$ https://en.wikipedia.org/wiki/Newline

${ }^{27}$ https://notepad-plus-plus.org/

${ }^{28}$ https://www.gnu.org/software/bash/manual/html_node/ Redirections.html
} 


\section{cat < myfile.txt}

The previous tr command used a new file for the standard output, because we cannot use the same file to read and write at the same time. To keep the same filename, we have to move the new file by using the mv command:

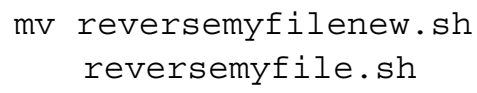

We can type man mv or mv --help to know more about this command line tool.

\section{Installing Tools}

These two last commands could be replaced by the dos 2 unix tool:

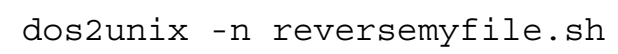

If not available, we have to install the dos 2 unix tool. For example, in the Ubuntu Windows Subsystem we need to execute:

\$ apt install dos2unix

The apt (Advanced Package Tool) command is used to install packages in many Linux systems $^{29}$. Another popular alternative is the yum (Yellowdog Updater, Modified) command ${ }^{30}$.

To avoid fixing line breaks each time we update our file when using Windows, a clearly better solution is to use a Unix friendly text editor.

When we are not using Windows, or we are using a Unix friendly text editor, the previous commands will execute but nothing will happen to the contents of reversemyfile.sh, since the tr command will not remove any character. To see the command working replace ' $\backslash r$ ' by ' $\$$ ' and check what happens.

\section{Permissions}

A script also needs permission to be executed, so every time we create a new script file we need to type:

\footnotetext{
${ }^{29}$ https://en.wikipedia.org/wiki/APT_(Debian)
}

${ }^{30}$ https://en.wikipedia.org/wiki/Yum_(software) chmod $u+x$ reversemyfile.sh

The command line tool chmod just gave the user (u) permissions to execute $(+x)$. We can type man chmod or chmod --help to know more about this command line tool.

Finally, we can execute the script by providing the myfile.txt as argument:

$$
\text { ./reversemyfile.sh myfile.txt }
$$

The contents of the file should appear in our terminal in the reverse order:

\section{line 4 \\ line 3 \\ line 2 \\ line 1}

Congratulations, we made our first script work $! \ddot{*}$

If we give more arguments, they will be ignored:

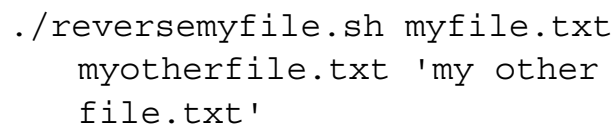

The output will be exactly the same because our script does not use $\$ 2$ and $\$ 3$, that in this case will represent myotherfile.txt and my other file.txt, respectively. We should note that when containing spaces, the argument must be enclosed by single quotes.

\section{Debug}

If something is not working well, we can debug the entire script by typing:

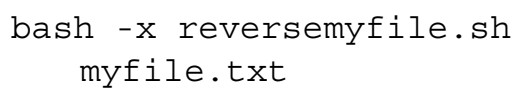

Our terminal will not only display the resulting text, but also the command line tools executed preceded by the plus character $(+)$ :

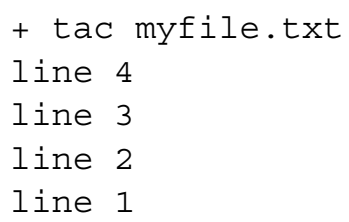


Alternatively, we can add the set $-\mathrm{x}$ command line in our script to start the debugging mode, and set $+\mathrm{x}$ to stop it.

\section{Save Output}

We can now save the output into another file named mynewfile.txt by typing:

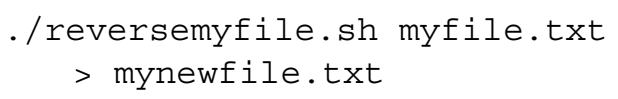

Again, to check if the file was really created, we can use the cat tool:

cat mynewfile.txt

Or, we can reverse it again by typing:

./reversemyfile.sh mynewfile. txt

Of course, the result should exactly be the original contents of myfile.txt.

\section{Web Identifiers}

The input argument(s) of our retrieval task is the chemical compound(s) of which we want to retrieve more information. For the sake of simplicity, we will start by assuming that the user knows the ChEBI identifier(s), i.e. the script does not have to search by the name of the compounds. Nevertheless, to find the identifier of a compound by its name is also possible, and this manuscript will describe how to do it later on.

So, the first step, is to automatically retrieve all proteins associated to the given input chemical compound, that in our example was caffeine (CHEBI:27732). In the manual process, we downloaded the files by manually clicking on the links shown as Export options, namely the URLs:

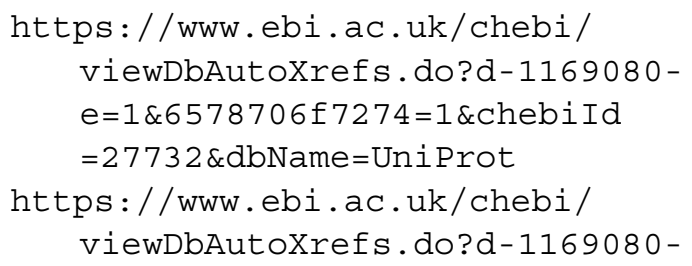

$\mathrm{e}=2 \& 6578706 \mathrm{f} 7274=1 \& \mathrm{chebi}$ Id

$=27732 \& \mathrm{dbName}=$ Uni Prot

https://www.ebi.ac.uk/chebi/

viewDbAutoXrefs.do?d-1169080-

$\mathrm{e}=3 \& 6578706 \pm 7274=1 \& \mathrm{chebi}$ Id

$=27732 \&$ dbName $=$ Uni Prot

for downloading a CSV, Excel, or XML file, respectively.

We should note that the only difference between the three URLs is a single numerical digit $(1,2$, and 3$)$ after the first equals character (=), which means that this digit can be used as an argument to select the type of file. Another parameter that is easily observable is the ChEBI identifier (27732). Try to replace 27732 by 17245 in any of those URLs by using a text editor, for example:

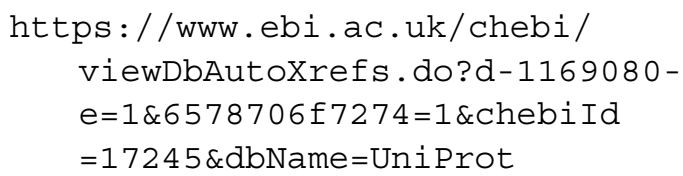

Now we can use this new URL in the internet browser, and check what happens. If we did it correctly, our browser downloaded a file with more than seven hundred proteins, since the 17245 is the ChEBI identifier of a popular chemical compound in life systems, the carbon monoxide.

In this case, we are not using a fully RESTful web service, but the data path is pretty modular and self-explanatory. The path is clearly composed of:

- the name of the database (chebi);

- the method (viewDbAutoXrefs.do);

- and a list of parameters and their value (arguments) after the question mark character (?).

The order of the parameters in the URL is normally not relevant. They are separated by the ampersand character (\&) and the equals character $(=)$ is used to assign a value to each parameter (argument). This modular structure of these URLs allows us to use them as data pipelines to fill our local files with data, like pipelines that transport oil or gas from one container to another. 


\section{Single and Double Quotes}

To construct the URL for a given ChEBI identifier, let us first understand the difference between single quotes and double quotes in a string (sequence of characters). We can create a script file named getproteins.sh by using a text editor to add the following lines:

1 echo 'The input: \$1'

2 echo "The input: \$1"

The command line tool echo displays the string received as argument. Do not forget to save it in our working directory and add the right permissions with chmod as we did previously with our first script.

Now to execute the script we will only need to type:

\section{/getproteins.sh}

The output on the terminal should be:

The input: $\$ 1$

The input:

This means that when using single quotes, the string is interpreted literally as it is, whereas the string within double quotes is analyzed, and if there is a special character, such as the dollar sign (\$), the script translates it to what it represents. In this case, $\$ 1$ represents the first input argument. Since no argument was given, the double quotes displays nothing.

To execute the script with an argument, we can type:

./getproteins.sh 27732

The output on our terminal should be:

The input: $\$ 1$

The input: 27732

We can check now that when using double quotes $\$ 1$ is translated to the string given as argument.

Now we can update our script file named getproteins.sh to contain only the following line:

1 echo "https://www.ebi.ac.uk/ chebi/viewDbAutoXrefs.do?d

$-1169080-e=1 \& 6578706 f 7274$

$=1 \&$ chebi $I d=\$ 1 \& d b$ Name $=$

UniProt"

\section{Comments}

Instead of removing the previous lines, we can transform them in comments by adding the hash character (\#) to the beginning of the line:

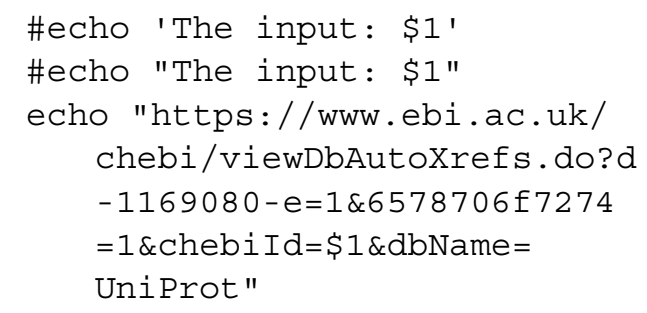

Commented lines are ignored by the computer when executing the script.

Now, we can execute the script giving the ChEBI identifier as argument:

\section{./getproteins.sh 27732}

The output on our terminal should be the link that returns the CSV file containing the proteins associated with caffeine.

\section{Data Retrieval}

After having the link, we need a web retrieval tool that works like our internet browser, i.e. receives as input a URL for programmatic access and retrieves its contents from the internet. We will use Client Uniform Resource Locator (cURL), which is available as a command line tool, and allows us to download the result of opening a URL directly into a file (man curl or curl - -help for more information).

For example, to display in our screen the list of proteins related to caffeine, we just need to add the respective URL as input argument:

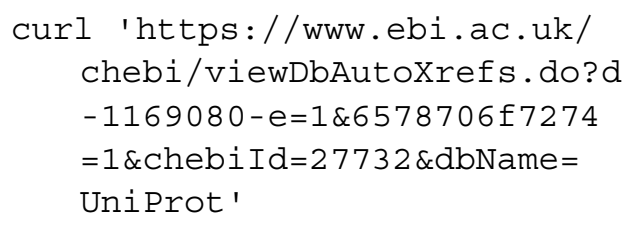

In some systems the curl command needs to be installed ${ }^{31}$. Since we are using a secure con-

31 apt install curl 
nection https, we may also need to install the $c a$ certificates package ${ }^{32}$.

An alternative to curl is the command wget, which also receives a URL as argument but by default wget writes the contents to a file instead of displaying it on the screen (man wget or wget - -help for more information). So, the equivalent command, is to add the - Ooption to select where the contents is placed:

wget -O- 'https://www.ebi.ac. uk/chebi/viewDbAutoXrefs. do?d-1169080-e=1\&6578706 f $7274=1 \&$ chebi $I d=27732 \&$ dbName=Uni Prot '

We should note that dash - character after - $\mathrm{O}$ represents the standard output. The equivalent long form to the -O option is --outputdocument=file.

The output on our terminal should be the long list of proteins:

\section{Q15413, Ryanodine receptor 3,CC - MISCELLANEOUS \\ Q92375, Thioredoxin reductase, DE Q92736, Ryanodine receptor 2,CC - MISCELLANEOUS}

Instead of using a fixed URL, we can update the script named getproteins.sh to contain only the following line:

1 curl "https://www.ebi.ac.uk/ chebi/viewDbAutoXrefs. do?d - $1169080-e=1 \& 6578706$ f7274 $=1 \&$ chebi Id $=\$ 1 \&$ dbName $=$ UniProt"

We should note that now we are using double quotes, since we replaced the caffeine identifier by $\$ 1$.

Now to execute the script we only need to provide a ChEBI identifier as input argument:

. /getproteins.sh 27732

The output on our terminal should be the long list of proteins:

32 apt install ca-certificates
Q15413, Ryanodine receptor 3, CC MISCELLANEOUS

Q92375, Thioredoxin reductase, DE

Q92736, Ryanodine receptor 2,CC MISCELLANEOUS

Or, if we want the proteins related to carbon monoxide, we only need to replace the argument:

. /getproteins.sh 17245

And the output on our terminal should be an even longer list of proteins:

...

Q58432, Phosphomethylpyrimidine

synthase, CC - CATALYTIC

ACTIVITY

Q62976, Calcium-activated

potassium channel subunit

alpha-1, CC - ENZYME

REGULATION; CC - DOMAIN

Q63185, Eukaryotic translation

initiation factor 2-alpha

kinase 1,CC - ENZYME

REGULATION

If we want to analyze all the lines we can redirect the output to the command line tool less, which allows us to navigate through the output by using the arrow keys. To do that we can add the bar character ( $\mid$ ) between two commands, which will transfer the output of the first command as input of the second:

\section{./getproteins.sh 27732 | less}

To exit from less just press q.

However, what we really want is to save the output as a file, not just printing some characters on the screen. Thus, what we should do is redirect the output to a CSV file. This can be done by adding the redirect operator $>$ and the filename, as described previously:

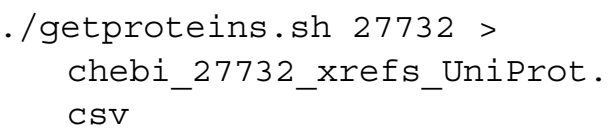

We should note that curl still prints some progress information into the terminal. 


\section{Standard Error Output}

This happens because it is displaying that information into the standard error output, which was not redirected to the file ${ }^{33}$. The $>$ character without any preceding number by default redirects the standard output. The same happens if we precede it by the number 1 . If we do not want to see that information, we can also redirect the standard error output (2), but in this case to the null device (/dev/null):

\section{./getproteins.sh $27732>$ chebi 27732 xrefs UniProt. Csv $2>/ \mathrm{dev} / \mathrm{null}$}

We can also use the - $\mathrm{s}$ option of curl in order to suppress the progress information, by adding it to our script file named getproteins.sh:

1 curl -s "https://www.ebi.ac.uk

/chebi/viewDbAutoXrefs.do?

$\mathrm{d}-1169080-e=1 \& 6578706 f 7274$

$=1 \&$ chebi $I d=\$ 1 \& d b$ Name $=$

UniProt"

The equivalent long form to the $-\mathrm{s}$ option is - -silent.

Now when executing the script, no progress information is shown:

./getproteins.sh $27732>$ chebi 27732 xrefs UniProt. CSV

To check if the file was really created and to analyze its contents, we can use the less command:

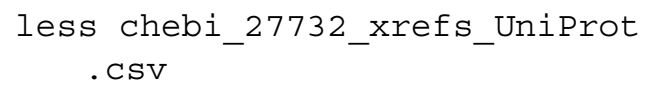

We can also open the file in our spreadsheet application, such as LibreOffice Calc or Microsoft Excel.

\footnotetext{
${ }^{33}$ https://www.gnu.org/software/bash/manual/html_node/ Redirections.html
}

As an exercise execute the script to get the CSV file with the associated proteins of water ${ }^{34}$ and gold ${ }^{35}$.

\section{Data Extraction}

Some data in the CSV file may not be relevant regarding our information need, i.e. we may need to identify and extract relevant data. In our case, we will select the relevant proteins (lines) using the command line tool grep, and secondly, we will select the column we need using the command line tool gawk, which is the GNU implementation of $a w k^{36}$. We should note that if we are using MobaXterm we may need to install the gawk package ${ }^{37}$. We can also replace gawk by awk in case another implementation is available ${ }^{38}$.

Since our information need is about diseases related to caffeine, we may assume that we are only interested in proteins that have one of these topics in the third column:

\section{CC - MISCELLANEOUS \\ CC - DISRUPTION PHENOTYPE \\ CC - DISEASE}

Extracting lines from a text file is the main function of grep. The selection is performed by giving as input a pattern that grep tries to find in each line, presenting only the ones where it was able to find a match. The pattern is the same as the one we normally use when searching for a word in our text editor. The grep command also works with more complex patterns such as regular expressions, that we will describe later on.

\footnotetext{
${ }^{34}$ https://www.ebi.ac.uk/chebi/searchId.do?chebiId= CHEBI: 15377

${ }^{35}$ https://www.ebi.ac.uk/chebi/searchId.do?chebiId= CHEBI:30050

${ }^{36}$ http://www.gnu.org/software/gawk/

${ }^{37}$ apt install gawk

${ }^{38}$ https://en.wikipedia.org/wiki/AWK\#

Versions_and_implementations
} 


\section{Single and Multiple Patterns}

We can execute the following command that selects the proteins with the topic CC MISCELLANEOUS, our pattern, in our CSV file:

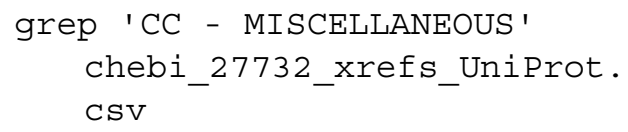

The output will be a shorter list of proteins, all with CC - MISCELLANEOUS as topic:

\section{A2AGL3, Ryanodine receptor 3,CC - MISCELLANEOUS \\ BOLPN4, Ryanodine receptor 2, CC - MISCELLANEOUS \\ E9PZQ0, Ryanodine receptor 1,CC - MISCELLANEOUS \\ E9Q401, Ryanodine receptor 2,CC - MISCELLANEOUS \\ F1LMY4, Ryanodine receptor 1,CC - MISCELLANEOUS \\ P11716, Ryanodine receptor 1,CC - MISCELLANEOUS \\ P21817, Ryanodine receptor 1,CC - DISEASE; CC - MISCELLANEOUS \\ P54867, Protein SLG1, CC - MISCELLANEOUS \\ Q9TS33, Ryanodine receptor 3, CC - MISCELLANEOUS \\ Q15413, Ryanodine receptor 3,CC - MISCELLANEOUS \\ Q92736, Ryanodine receptor 2,CC - MISCELLANEOUS}

To use multiple patterns, we must precede each pattern with the -e option:

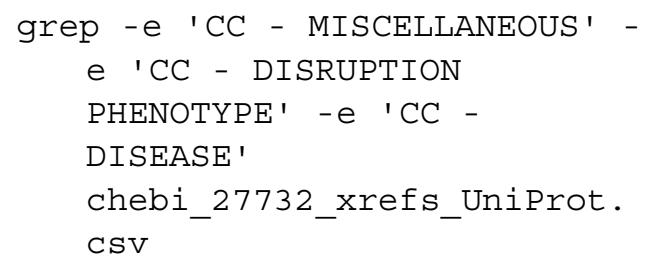

The equivalent long form to the -e option is - regexp=PATTERN.

The output on our terminal should be a longer list of proteins:

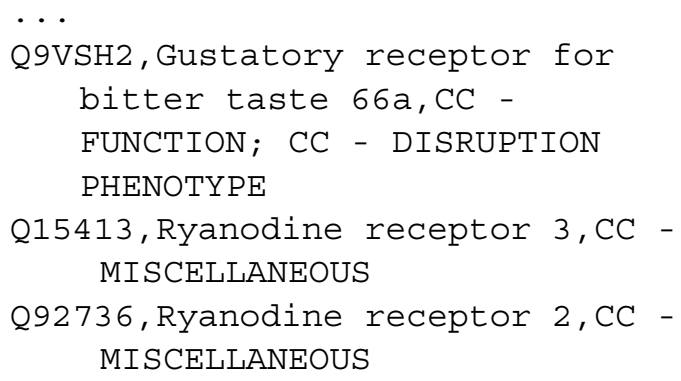

We should note that as previously, we can add | less to check all of them more carefully. The less command also gives the opportunity to find lines based on a pattern. We only need to type / and then a pattern.

We can now update our script file named getproteins.sh to contain the following lines:

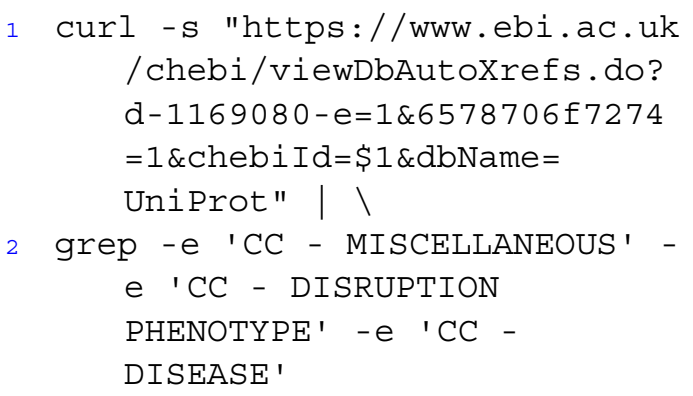

We should note that we added the - $\mathrm{s}$ option to suppress the progress information of curl, and the characters $\mid \backslash$ to the end of line to redirect the output of that line as input of the next line, in this case the grep command. We need to be careful in ensuring that $\backslash$ is the last character in the line, i.e. spaces in the end of the line may cause problems.

We can now execute the script again:

$$
\text { ./getproteins.sh } 27732
$$

The output should be similar of what we got previously, but the script downloads the data and filters immediately.

To save the file with the relevant proteins, we only need to add the redirection operator:

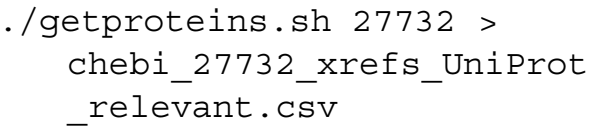




\section{Data Elements Selection}

Now we need to select just the first column, the one that contains the protein identifiers. Selecting columns from a tabular file is one easy task for gawk, that besides performing pattern scanning also provides a complex processing language $\left(\mathrm{AWK}^{39}\right)$. This processing language can be highly complex ${ }^{40}$ and it is out of our scope for this introductory manuscript. The gawk command can receive as arguments the character that divides each data element (column) in a line using the $-F$ option, and an instruction of what to do with it enclosed by single quotes and curly brackets. The equivalent long form to the $-F$ option is - $f i e l d-$ separator $=f s$.

For example, we can get the first column of our CSV file:

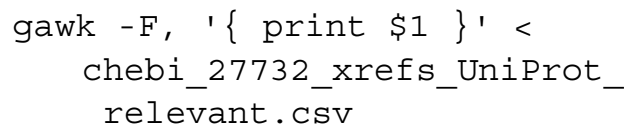

We should note that comma (, ) is the character that separates data elements in a CSV file, and that print is equivalent to echo, and $\$ 1$ represents the first data element.

The command will display only the first column of the file, i.e. the protein identifiers:

...

Q9VSH2

Q15413

Q92736

For example, we can get the first and third columns separated by a comma:

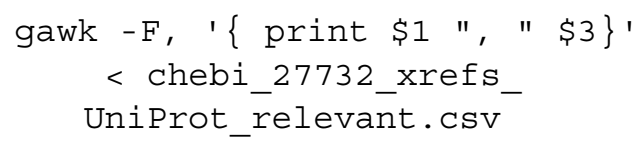

Now, the output contains both the first and third column of the file:

-..

Q9VSH2, CC - FUNCTION; CC DISRUPTION PHENOTYPE

Q15413, CC - MISCELLANEOUS

\footnotetext{
${ }^{39}$ https://en.wikipedia.org/wiki/AWK

${ }^{40} \mathrm{https}$ //www6.software.ibm.com/developerworks/ education/au-gawk/au-gawk-a4.pdf
}

Q92736, CC - MISCELLANEOUS

We can update our script file named getproteins.sh to contain the following lines:

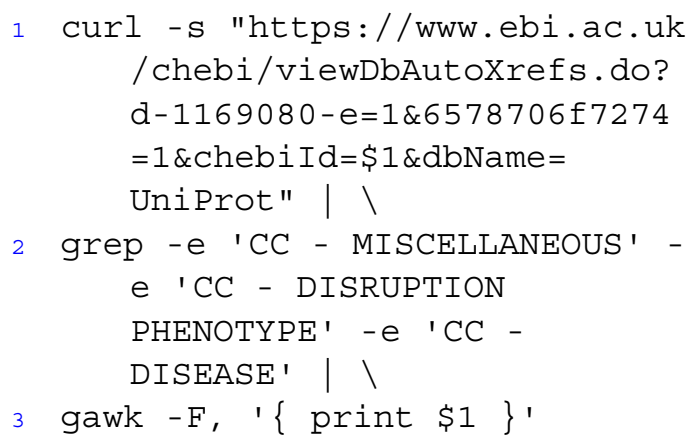

The last line is the only that changes, except the $\mid \backslash$ in the previous line to redirect the output.

To execute the script, we can type again:

. /getproteins.sh 27732

The output should be similar of what we got previously, but now only the protein identifiers are displayed.

To save the output as a file with the relevant proteins' identifiers, we only need to add the redirection operator:

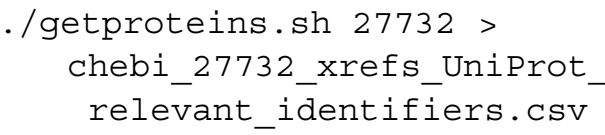

\section{Task Repetition}

Given a protein identifier we can construct the URL that will enable us to download its information from UniProt. We can use the RESTful web services provided by UniProt ${ }^{41}$, more specifically the one that allow us to retrieve a specific entry ${ }^{42}$. The construction of the URL is simple, it starts always by https://www .uniprot.org/uniprot/, followed by the protein identifier, ending with a dot and the data format. For example, the link for protein P21817 using the XML format is: http://www.uniprot. org/uniprot/P21817.xml

\footnotetext{
${ }^{41}$ https://www.uniprot.org/help/api

${ }^{42}$ https://www.uniprot.org/help/api_retrieve_entries
} 


\section{Assembly Line}

However, we need to construct one URL for each protein from the list we previously retrieved. The size of the list can be large (hundreds of proteins), varies for different compounds and evolves with time. Thus, we need an assembly line in which a list of proteins identifiers, independently of its size, are added as input to commands that construct one URL for each protein and retrieve the respective file.

The xargs command line tool works as an assembly line, it executes a command per each line given as input. We should note that if we are using MobaXterm we may need to install the findutils package ${ }^{43}$, since the default xargs only has minimal options ${ }^{44}$.

We can start by experimenting the xargs command by giving as input the list of protein identifiers in file chebi_27732_xrefs_UniProt_ relevant_identifiers.csv, display each identifier on the screen in the middle of a text message by providing the echo command as argument:

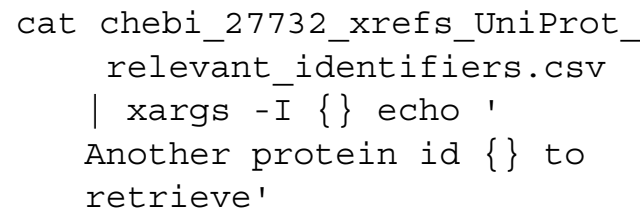

The xargs command received as input the contents our CSV file, and for each line displayed a message including the identifier in that line. The - I option tells xargs to replace \{\} in the command line given as argument by the value of the line being processed. The equivalent long form to the $-I$ option is - - replace $=R$.

The output should be something like this:

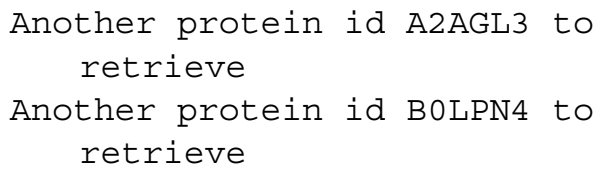

\footnotetext{
${ }^{43}$ apt install findutils

${ }^{44}$ In some versions the scripts may have to use xargs . exe to invoke the new version. Or rename the xargs shortcut in the bin folder to other name, that way the right version will always be invoked.
}

Another protein id E9PZQ0 to retrieve

Instead of creating inconsequential text messages, we can use xargs to create the URLs:

$$
\begin{aligned}
& \text { cat chebi_27732_xrefs_UniProt } \\
& \text { relevant_identifiers.csv } \\
& \text { | xargs -I \{\} echo 'https } \\
& \text { ://www.uniprot.org/uniprot } \\
& \text { / }\{\} . x m l '
\end{aligned}
$$

The output should be something like this:

https: //www. uniprot.org/uniprot/ A2AGL3 . xml

https: //www. uniprot.org/uniprot/

BOLPN4 . Xml

https://www. uniprot.org/uniprot/

E9PZQ0.xml

We can try to use these links in our internet browser to check if those displayed URLs are working correctly.

Now that we have the URLs, we can automatically download the files using the curl command instead of echo:

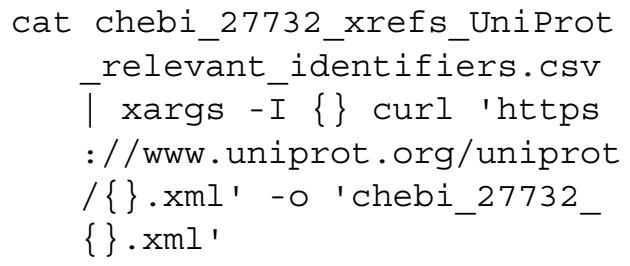

We should note that we now use the - o option to save the output to a given file, named after each protein identifier. The equivalent long form to the - o option is --output <file>.

To check if everything worked as expected we can use the Is command to view which files were created:

Is chebi_27732_*.xml

The asterisk character $(*)$ character is here used to represent any file whose name starts with chebi_27732_and ends with .xml.

To check the contents of any of them, we can use the less command:

less chebi_27732_P21817.xml 


\section{File Header}

We should note that the content of every file has to start with < ? xml otherwise there was a download error, and we have to run curl again for those entries. To check the header of each file, we can use the head command together with less.

\section{\$ head -n 1 chebi_27732_*.xml | less}

The - $\mathrm{n}$ option specifies how many lines to print, in the previous command just one.

If for any reason, we are not able to download the files from UniProt, we can get them from the book file archive ${ }^{45}$.

\section{Variable}

We can now update our script file named getproteins.sh to contain the following lines:

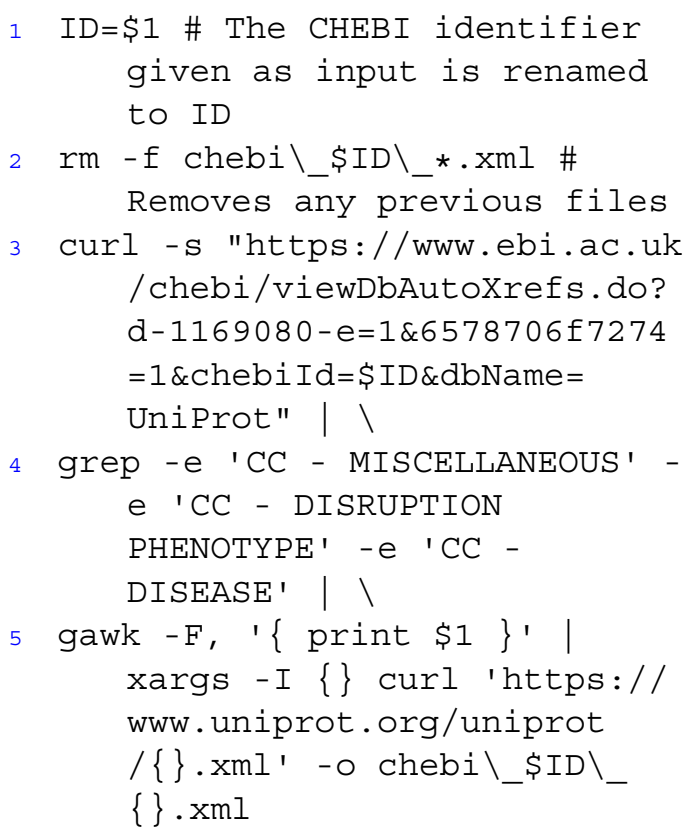

We should note that the last line now includes the xargs and curl commands, and the \$ID variable. This new variable is created in the first line to contain the first value given as argument

${ }^{45}$ http://labs.rd.ciencias.ulisboa.pt/book/
(\$1). So, every time we mention \$ID in the script we are mentioning the first value given as argument. This avoids ambiguity in cases where \$1 is used for other purposes, like in the gawk command. Since the preceding character of \$ID is an underscore (_), we have to add a backslash $(\backslash)$ before it. The second line uses the rm command to remove any files that were downloaded in a previous execution. We also now added two comments after the hash character, so we humans do not forget why these commands are needed for.

To execute the script once more:

./getproteins.sh 27732

And again, to check the results:

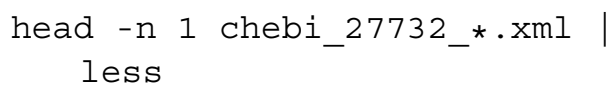

\section{XML Processing}

Assuming that our information need only concerns human diseases, we have to process the XML file of each protein to check if it represents a Homo sapiens (Human) protein.

\section{Human Proteins}

For performing this filter, we can again use the grep command, to select only the lines of any XML file that specify the organism as Homo sapiens:

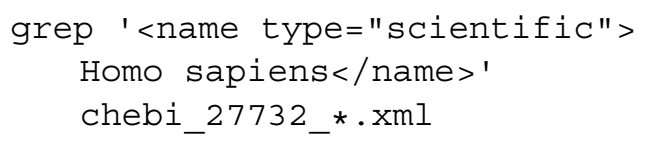

We should get in our display the filenames that represent a human protein, i.e. something like this:

Chebi_27732_P21817.xml:<name

type= "scientific" $>$ Homo

sapiens</name>

chebi_27732_Q15413.xml:<name

type="scientific" $>$ Homo

sapiens</name> 


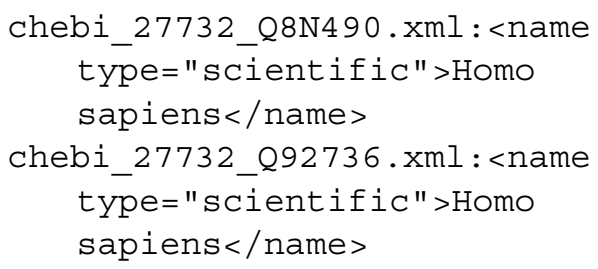

We should note that since the asterisk character (*) provides multiple files as argument to grep, the ones whose name starts with chebi_27732_ and ends with .xml, the output now includes the filename (followed by a colon) where each line was matched.

We can use the gawk command to extract only the filename, but grep has the -1 option to just print the filename:

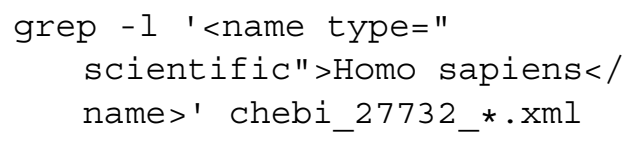

The equivalent long form to the -1 option is - files-with-matches.

The output will now show only the filenames:

chebi_27732_P21817.xml

Chebi_27732_Q15413.xml

chebi_27732_Q8N490.xml

chebi_27732_Q92736.xml

These four files represent the four Human proteins related to caffeine.

\section{PubMed Identifiers}

Now we need to extract the PubMed identifiers from these files to retrieve the related publications. For example, if we execute the following command:

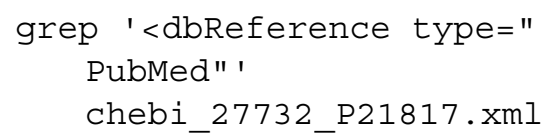

The output is a long list of publications related to protein $P 21817$ :

$<$ dbReference type="PubMed" id=" $2298749 " />$

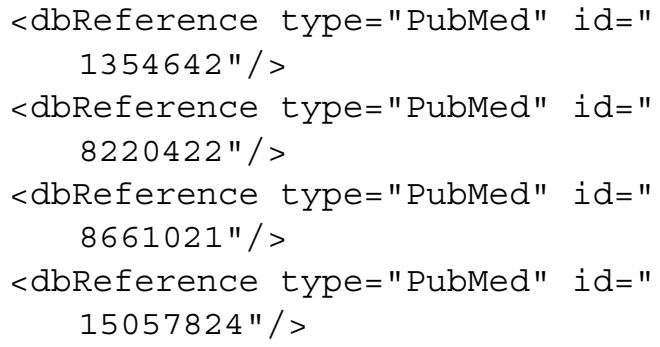

To extract just the identifier, we can again use the gawk command:

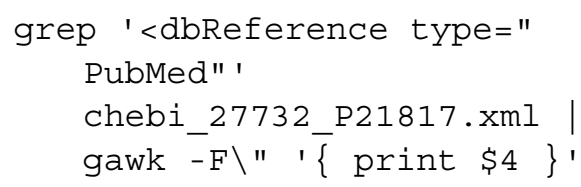

We should note that " is used as the separation character and, since the PubMed identifier appears after the third ", the $\$ 4$ represents the identifier.

Now the output should be something like this:

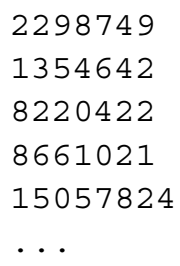

\section{PubMed Identifiers Extraction}

Now to apply to every protein we may again use the xargs command:

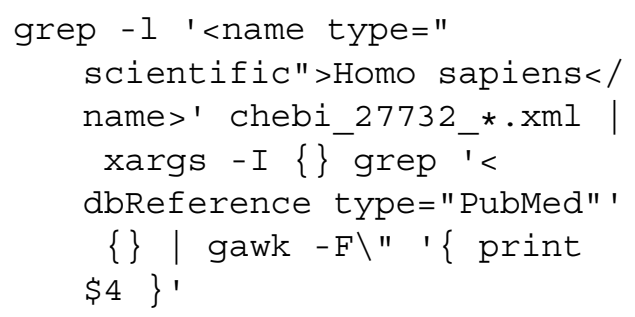

This may provide a long list of PubMed identifiers, including repetitions since the same publication can be cited in different entries. 


\section{Duplicate Removal}

To help us identify the repetitions, we can add the sort command (man sort or sort -help for more information), which will display the repeated identifiers in consecutive lines (due by sorting all identifiers):

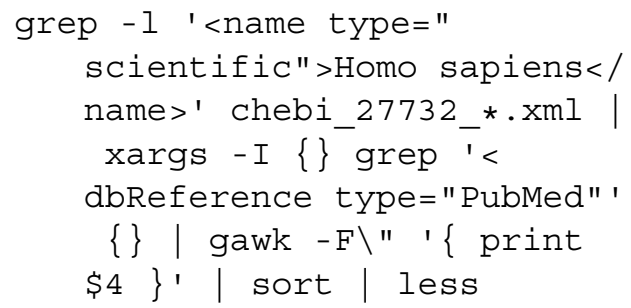

For example some repeated PubMed identifiers that we should easily be able to see:

10051009

10051009

10097181

10097181

10484775

10484775

...

Fortunately, we also have the -u option that removes all these duplicates:

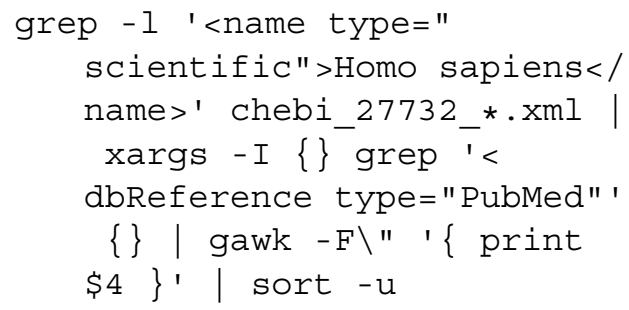

To easily check how many duplicates were removed, we can use the word count wc command with and without the usage of the -u option:

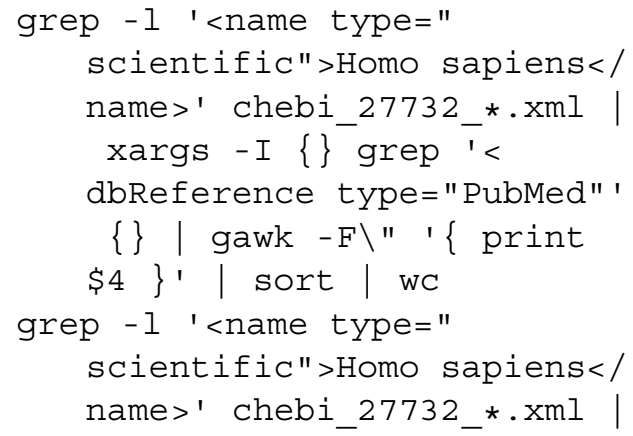

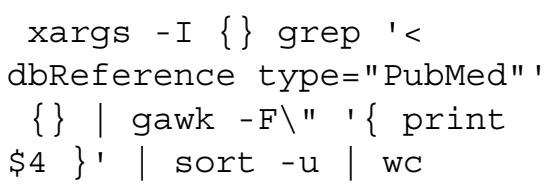

In case we have in our folder any auxiliary file, such as chebi_27732_P21817_entry .xml, we should add the option --exclude *entry.xml to the first grep command.

The output should be something like:

$\begin{array}{lll}255 & 255 & 2243\end{array}$

1291291136

wC prints the numbers of lines, words, and bytes, thus in our case we are interested in first number (man wC or wc --help for more information). We can see that we have removed $255-129=126$ duplicates.

Just for curiosity, we can also use the shell to perform simple mathematical calculations using the expr command:

expr 255 - 129

Now let us create a script file named getpublications.sh by using a text editor to add the following lines:

1 ID=\$1 \# The CHEBI identifier given as input is renamed to ID

2 grep -1 '<name type=" scientific">Homo sapiens $<$ / name>' chebi \_\$I \_*.xml | $\backslash$

3 xargs -I \{\} grep ' $<$ dbReference type $=$ "PubMed" ' \{\} $\mid 1$

4 gawk $-F \backslash "$ ' $\{$ print $\$ 4\}$ '। sort $-u$

Again, do not forget to save it in our working directory, and add the right permissions with chmod as we did previously with the other scripts.

To execute the script again:

\section{./getpublications.sh 27732}

We can verify how many unique publications were obtained by using the -1 option of wc, that provides only the number of lines: 


\section{. /getpublications.sh 27732 wC -1}

The output will be 129 as expected.

\section{Complex Elements}

Not always the XML elements are in the same line, as fortunately was the case of the PubMed identifiers. In those cases, we may have to use the xmllint command, a parser that is able to extract data through the specification of a XPath query, instead of using a single line pattern as in grep.

\section{XPath}

XPath (XML Path Language) is a powerful tool to extract information from XML and HTML documents by following their hierarchical structure. Check W3C for more about XPath syntax ${ }^{46}$. We should note that xmllint may not be installed by default depending on our operating system, but it should be very easy to do it ${ }^{47}$ If we are using MobaXterm, then we need to install the xmllint plugin ${ }^{48}$.

\section{Namespace Problems}

In the case of our protein XML files, we can see that their second line defines a specific namespace using the xmlns attribute ${ }^{49}$ :

<uniprot xmlns="http://uniprot . org/uniprot" xmlns:xsi="http: //www.w3 .org/2001/XMLSchemainstance" xsi:schemaLocation= "http://uniprot.org/uniprot http://www. uniprot.org/ support/docs/uniprot.xsd" >

\footnotetext{
${ }^{46}$ https://www.w3schools.com/xml/xpath_syntax.asp

${ }^{47}$ apt install libxml2-utils

${ }^{48}$ https://mobaxterm.mobatek.net/plugins.html

${ }^{49}$ https://www.w3schools.com/xml/xml_namespaces.asp
}

This complicates our XPath queries, since we need to explicitly specify that we are using the local name for every element in a XPath query. For example, to get the data in each reference element:

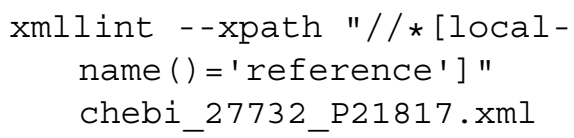

We should note that // means any path in the XML file until reaching a reference element. The square brackets in XPath queries normally represent conditions that need to be verified.

\section{Only Local Names}

If we are only interested in using local names there is a way to avoid the usage of local -name () for every element in a XPath query. We can identify the top-level element, in our case entry, and extract all the data that it encloses using a XPath query. For example, we can create the auxiliary file chebi_27732_P21817_entry.xml by adding the redirection operator:

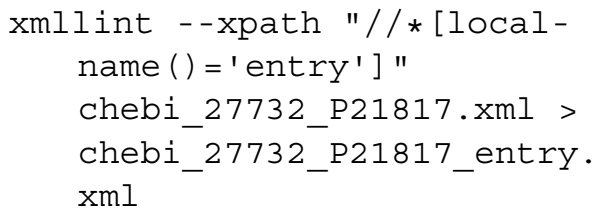

The new XML file now starts and ends with the entry element without any namespace definition:

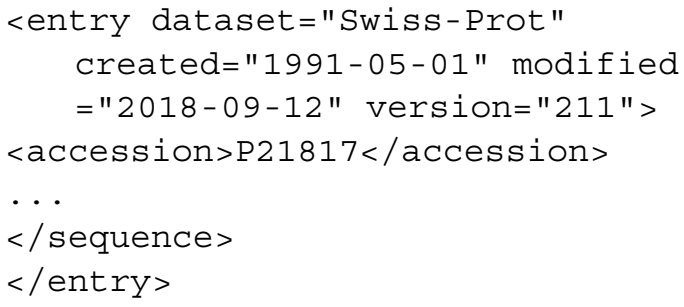

Now we can apply any XPath query, for example / / reference, on the auxiliary file without the need to explicitly say that it represents a local name: 


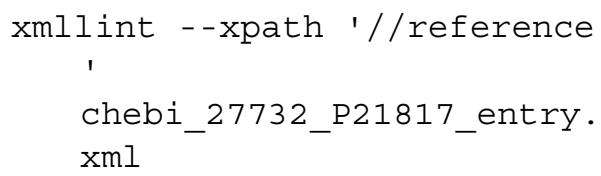

The output should contain only the data inside of each reference element:

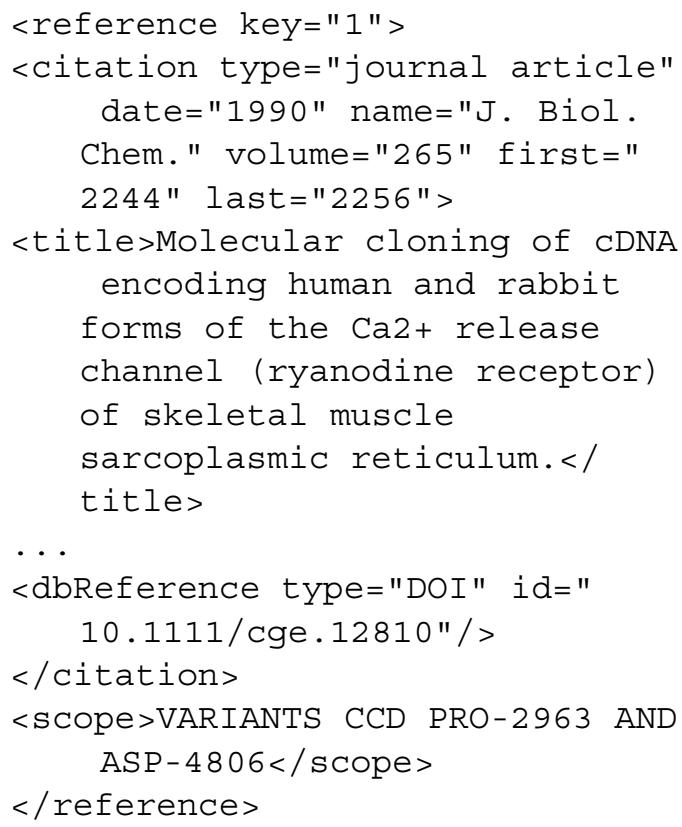

\section{Queries}

The XPath syntax allow us to create many useful queries, such as:

- //dbReference - elements of type $d b R e f e r e n c e$ that are descendants of something; Result:

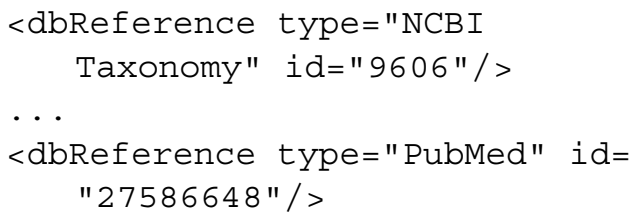

- /entry//abReference - equivalent to the previous query but specifying that the dbReference elements are descendants of the entry element;
- /entry/reference/citation/dbRe ference- equivalent to the previous query but specifying the full path in the XML file;

- //abReference/* - any child elements of a dbReference element; Result:

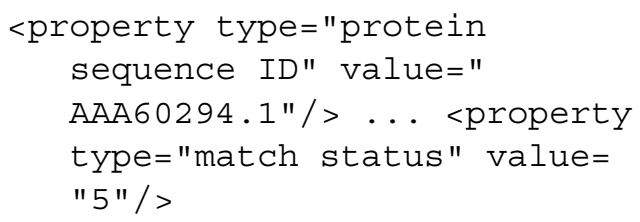

- //dbReference/property [1] - first property element of each dbReference element; Result:

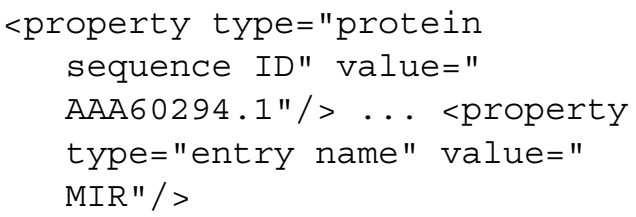

- //dbReference/property [2] - second property element of each dbReference element; Result:

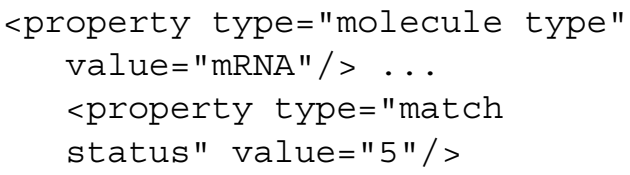

- //dbReference/property [3] - third property element of each dbReference element; Result:

<property type="molecule type" value $=$ "Genomic_DNA" $/>$. . . <property type="project" value="UniProtKB" / >

- //abReference/property/@type all type attributes of the property elements; Result:

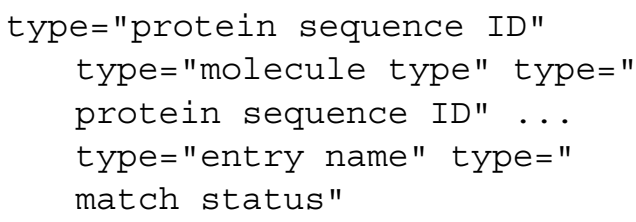

- //dbReference/property [@type=" protein sequence ID"] - the previous property elements that have an attribute type equal to protein sequence ID; Result: 
$<$ property type="protein

sequence ID" value="

AAA60294.1"/> ... <property

type="protein sequence ID"

value= "ENSP00000352608"/>

- //dbReference/property [@type=" protein sequence ID"]/@value the string assigned to each attribute value of the previous property elements; Result:

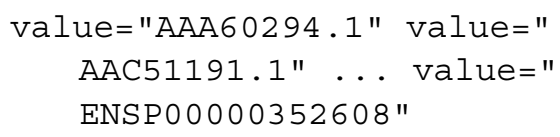

- //sequence/text () - the contents inside the sequence elements; Result:

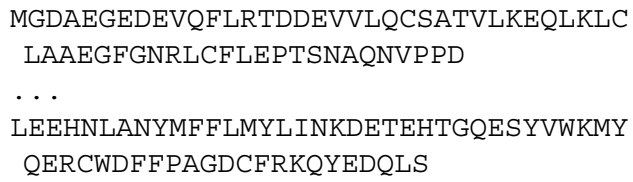

We should note that to try the previous queries we only need to replace the string after the -xpath option of the previous xmllint command, such as:

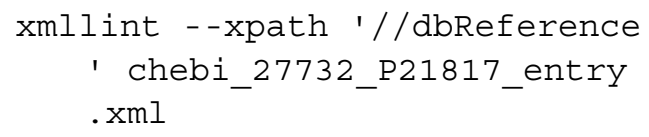

Thus, an alternative way to extract the PubMed identifiers using xmllint instead of grep, would be something like this:

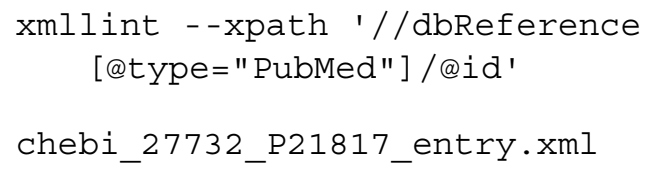

However, the output contains all identifiers in the same line and with the id label:

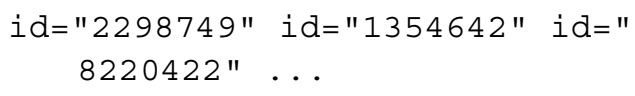

\section{Extracting XPath Results}

To extract the identifiers, we need to apply the $t r$ command to split the output in multiple lines (one line per identifier), and then the gawk command:

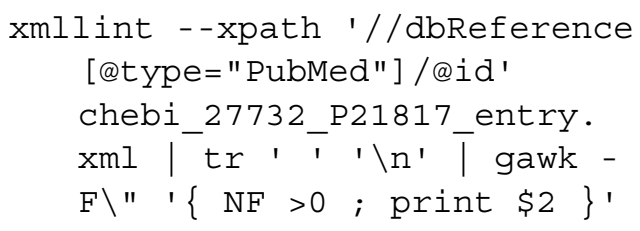

The tr command replaces each space by a newline character, and the gawk command extracts the value inside the double quotes. We should note that NF $>0$ is used to only select lines with at least a separation character ", i.e. in our case it ignores empty lines.

\section{Text Retrieval}

Now that we have all the PubMed identifiers, we need to download the text included in the titles and abstracts of each publication.

\section{Publication URL}

To retrieve from the UniProt citations service the publication entry of a given identifier, we can again use the curl command and a link to the publication entry. For example, if we click on the Format button of the UniProt citations service entry $^{50}$, we can get the link to the RDF/XML version. $\mathrm{RDF}^{51}$ is a standard data model that can be serialized in a XML format. Thus, in our case, we can deal with this format like we did with XML.

We can retrieve the publication entry by executing the following command:

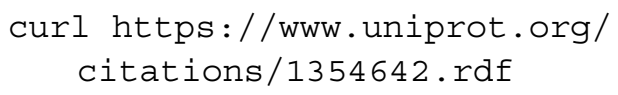

Thus, we can now update the script getpublications.sh to have the following commands:

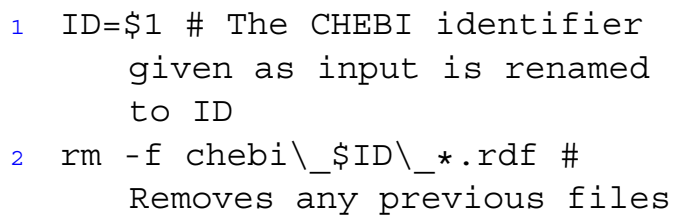

\footnotetext{
${ }^{50} \mathrm{https} / / /$ www.uniprot.org/citations/1354642

${ }^{51} \mathrm{https} / / / \mathrm{www} . w 3 . o r g / R D F /$
} 


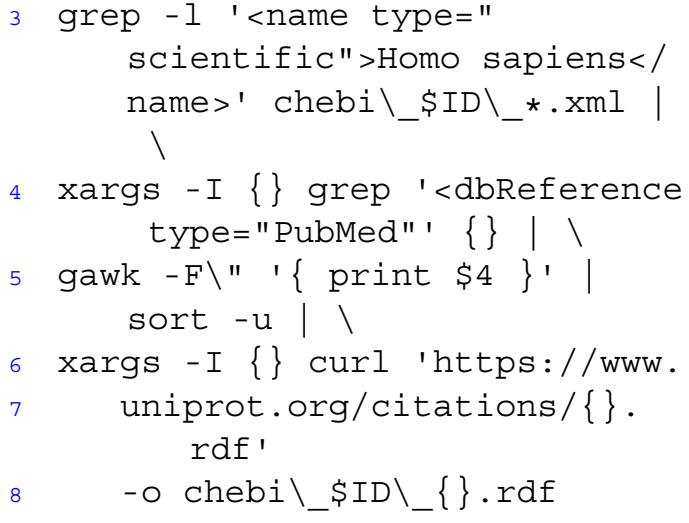

We should note that only the second and last lines were updated to remove and retrieve the files, respectively.

Now let us execute the script:

\section{./getpublications.sh 27732}

It may take a while to download all the entries, but probably no more than one minute with a standard internet connection.

To check if everything worked as expected we can use the Is command to view which files were created:

Is chebi_27732_*.rdf

If for any reason, we are not able to download the abstracts from UniProt, we can get them from the book file archive ${ }^{52}$.

\section{Title and Abstract}

Each file has the title and abstract of the publication as values of the title and rdfs:comment elements, respectively. To extract them we can again use the grep command:

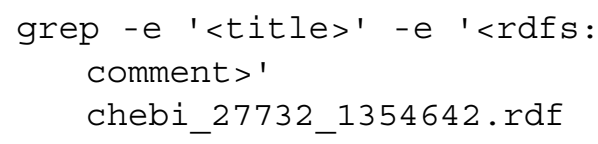

The output should be something like these two lines:

$$
\begin{array}{r}
<\text { title }>\text { Polymorphisms ... } \\
\text { hyperthermia.</title }
\end{array}
$$

$<$ rdfs:comment >Twenty-one ...

gene.</rdfs: comment >

To remove the XML elements, we can again use gawk:

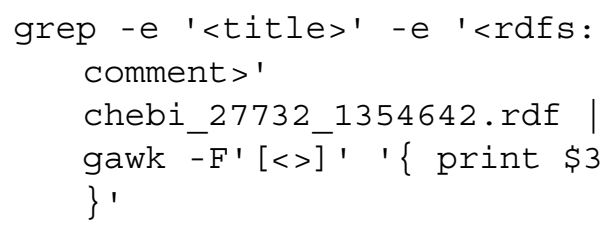

We should note that we now use two characters as field separators $<$ and $>$ to get the text between the first $>$ and the second <. The first field separator is $<$ so $\$ 2$ contains the string tit le or rdf $\mathrm{s}$ : comment while $\$ 1$ is empty. The second field separator is > so $\$ 3$ contains the string we want to keep.

The output should now be free of XML elements:

Polymorphisms ... hyperthermia.

Twenty-one ... gene.

Thus, let us create the script gettext.sh to have the following commands:

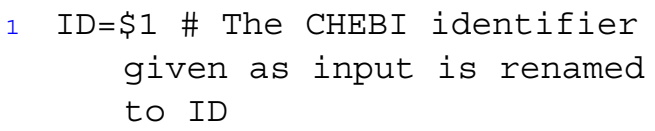

Again do not forget to save it in our working directory, and add the right permissions.

Now to execute the script and see the retrieved text:

./gettext.sh 27732 | less

We can save the resulting text in a file named chebi_27732.txt that we may share or read using our favorite text editor, by adding the redirection operator:

./gettext.sh $27732>$ chebi_27732.txt

${ }^{52} \mathrm{http} / / /$ labs.rd.ciencias.ulisboa.pt/book/ 


\section{Disease Recognition}

Instead of reading all that text to find any disease related with caffeine, we can try to find sentences about a given disease by using grep:

\section{' grep 'malignant hyperthermia' chebi 27732 .txt}

To save the filtered text in a file named chebi_27732_hyperthermia.txt, we only need to add the redirection operator:

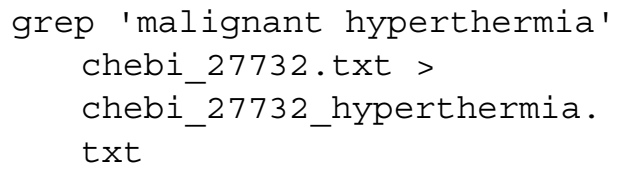

This is a very simple way of recognizing a disease in text. The next chapters will describe how to perform more complex text processing tasks.

\section{Further Reading}

If we really want to become an expert in shell scripting we may be interested in reading a book specialized in the subject, such as the book entitled The Linux command line: a complete introduction (Shotts Jr 2012).

A more pragmatic approach is to explore the vast number of online tutorials about shell scripting and web technologies, such as the ones provided by W3Schools ${ }^{53}$.

\footnotetext{
${ }^{53}$ https://www.w3schools.com/
}

Open Access This chapter is licensed under the terms of the Creative Commons Attribution 4.0 International License (http://creativecommons.org/licenses/by/4.0/), which permits use, sharing, adaptation, distribution and reproduction in any medium or format, as long as you give appropriate credit to the original author(s) and the source, provide a link to the Creative Commons licence and indicate if changes were made.

The images or other third party material in this chapter are included in the chapter's Creative Commons licence, unless indicated otherwise in a credit line to the material. If material is not included in the chapter's Creative Commons licence and your intended use is not permitted by statutory regulation or exceeds the permitted use, you will need to obtain permission directly from the copyright holder. 


\section{Text Processing}

\begin{abstract}
In the previous chapter we were able to automatically process structured data to retrieve biomedical text about any chemical compound, such as caffeine. This chapter will provide a step-by-step introduction to how we can process that text using shell script commands, specifically extract information about diseases related to caffeine. The goal is to equip the reader with an essential set of skills to extract meaningful information from any text.
\end{abstract}

\section{Keywords}

NLP: Natural Language Processing · Text mining $\cdot$ Pattern matching · String matching · Word matching · Evaluation metrics . Regular expressions - Tokenization - NER: Named-Entity Recognition $\cdot$ Relation extraction

In the previous chapter we were able to automatically process structured data to retrieve biomedical text about any chemical compound, such as caffeine. This chapter will provide a step-bystep introduction to how we can process that text using shell script commands, specifically extract information about diseases related to caffeine. The goal is to equip the reader with an essential set of skills to extract meaningful information from any text.

\section{Pattern Matching}

We used the grep command in the last chapter to find a disease in the text, since grep receives as argument a pattern to find an exact match in the text, like any search functionality provided by conventional text editors. However, we may need to search for multiple patterns even when interested in a single disease. For example, when searching for mentions of malignant hyperthermia, we may also be interested in finding mentions using related expressions, such as:

\section{MH - acronym \\ MHS - acronym for malignant hyperthermia susceptible}

Since we already know how to deal with multiple patterns by using the -e option, we may easily solve this problem by executing:

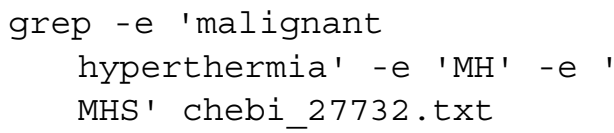

\section{Case Insensitive Matching}

When dealing with text, using a case sensitive search is usually a good approach to avoid wrong matches. For example, acronyms are normally in upper case, while the full name is usually in lowercase having sometimes the first letter of 
each word (or only the first word) in uppercase. So, instead of using a full case sensitive grep, we might think on performing a case sensitive grep for the acronyms and a case insensitive grep for the disease words using the - $i$ option:

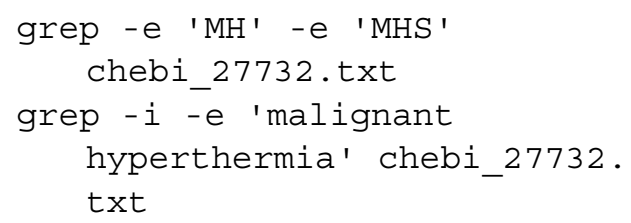

The equivalent long form to the $-i$ option is --ignore-case. We should note that each execution of grep will produce two separate lists of matching lines that might be overlapped.

Alternatively, we can also convert it to just one case sensitive grep, if we are sure that Malignant hyperthermia is the only alternative case to malignant hyperthermia present in the text. So, we can add it as another pattern:

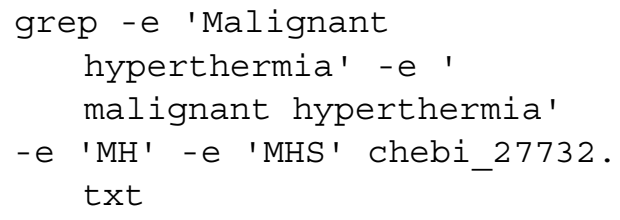

\section{Number of Matches}

To be sure that we are not losing any match, we can count the number of matching lines for both cases. First we execute a case insensitive grep and then we execute a case sensitive grep, both using the $-\mathrm{C}$ option:

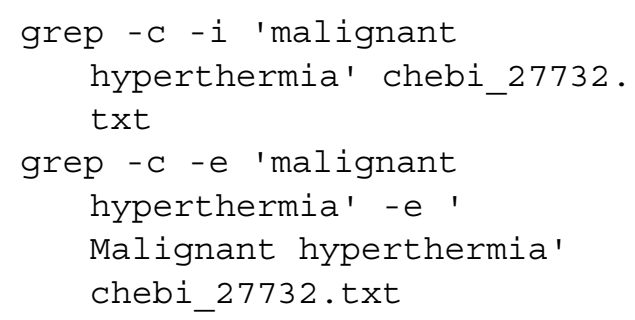

The equivalent long form to the $-\mathrm{c}$ option is - count.

In our case, the output should show 96 and 95 matching lines for the insensitive and sensitive patterns, respectively.
This means that there is a line that is not caught by the case sensitive pattern. To identify which one is, we can manually analyze each of the 96 matching lines one by one. But the goal of this book is exactly avoiding these type of tedious tasks. One thing we can do to solve this issue is to find from the case insensitive matches the one that do not match the case sensitive patterns.

\section{Invert Match}

Fortunately, the grep command has the -v option that inverts the matching and returns the lines of text that do not contain any matching. The equivalent long form to the $-\mathrm{v}$ option is - invert-match.

Thus, if we apply the inverted match with the case sensitive patterns to the output given by the case insensitive matching, we will get our outlier mention:

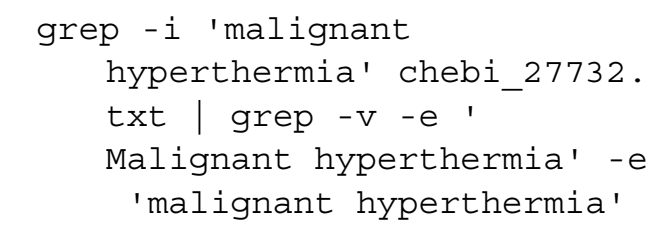

From the output, we can easily identify the missing matching line:

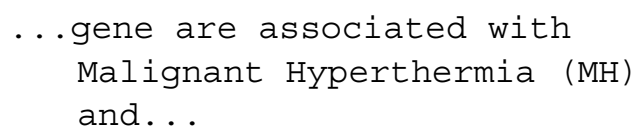

We were missing the case where both words have the first letter in uppercase.

Thus, to obtain all the matching lines in a case sensitive match we just have to include the missing match as another pattern:

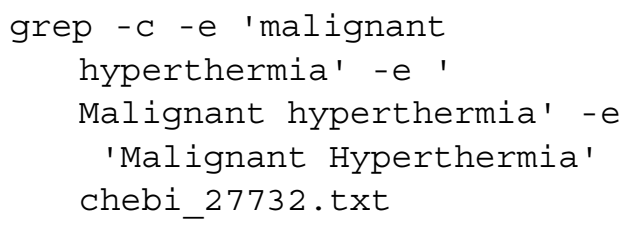

\section{File Differences}

Another alternative to compare different matches, is to use the diff command that 
receives as input two files and identifies their differences. So, we can create two auxiliary files and then apply the diff to them:

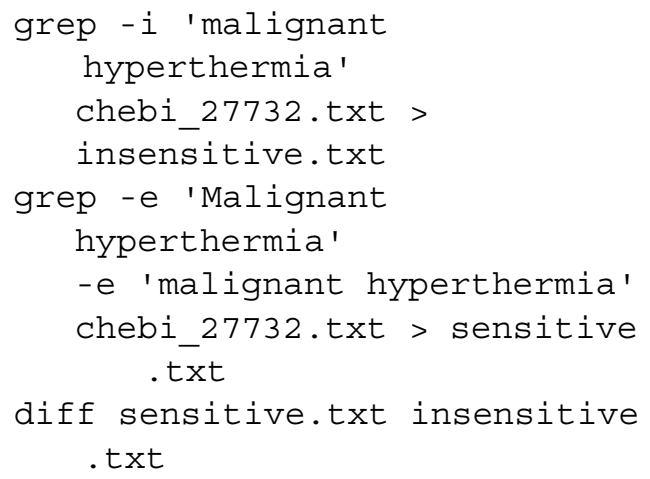

The output should be the same text.

A problem that may occur with case sensitive matching is that some acronyms are defined with lowercase letters in the middle, such as ChEBI, and humans are not consistent with the way they mention them. The same acronym may be mentioned in their original form or with all letters in uppercase, or just some of them. Moreover, these inconsistent mentions sometimes may even be found in the same publication. We hope not in this book! $\ddot{\sim}$

\section{Evaluation Metrics}

These inconsistencies made by humans when mentioning case sensitive expressions, is one of the reasons that most online search engines use case insensitive searches as default. This type of approach favors recall, while case sensitive search favor precision ${ }^{1}$.

Recall is the proportion of the number of correct matches found by our tool over the total number of correct mentions in the texts (found or not found). Case insensitive searches avoid missing mentions, so they favor recall.

Precision is the proportion of the number of correct matches found by our tool over the total number of matches found (correct or incorrect). Case sensitive searches avoid incorrect matches, so they favor precision.

\footnotetext{
${ }^{1}$ https://en.wikipedia.org/wiki/Precision_and_recall
}

Normally, there is a trade-off between precision and recall. Using a technique that improves precision, most of the times, will decrease recall, and vice-versa. To know how good the trade-off is, we can use the F-measure, which is the harmonic average of the precision and recall $^{2}$.

\section{Word Matching}

Acronyms (or terms) may also appear inside common words or longer acronyms. For example, when searching for $M H$, the word victimhood will produce a match:

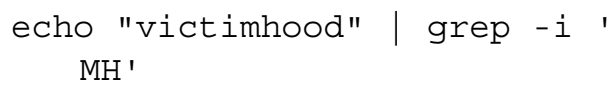

The problem with victimhood could be easily solved by using case sensitive matching, but not for a longer acronym. For example, the acronym NEDMHM for neurodevelopmental disorder with midbrain and hindbrain malformations will produce a case sensitive match:

\section{echo "NEDMHM" | grep 'MH'}

One way to address this problem is to use the -w option of grep to only match entire words, i.e. the match must be preceded and followed by characters that are not letters, digits, or an underscore (or be at the beginning or end of the line). The equivalent long form to the $-\mathrm{w}$ option is - -word-regexp.

Using this option, neither victimhood or NEDMHM will produce a match:

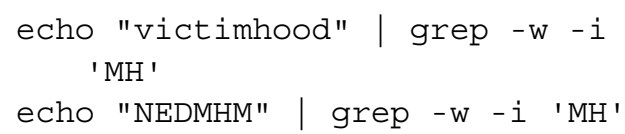

Word matching improves precision but decreases recall, since we may miss some less common acronyms that we are not aware of, but are still relevant for our study. For example, consider that we may also be interested in the following acronyms:

\footnotetext{
${ }^{2}$ https://en.wikipedia.org/wiki/F1_score
} 
MHE - acronym for malignant hyperthermia equivocal

MHN - acronym for malignant hyperthermia normal

If we apply word matching, we will not get a match, since both exact matches are followed by a letter:

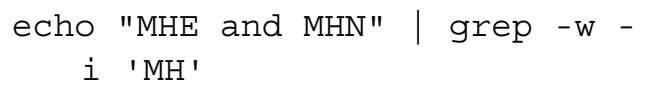

These are not trivial problems to solve by exact pattern matching, we may need regular expressions to address some of these issues more efficiently.

\section{Regular Expressions}

When dealing with natural language text we may need more flexibility than the one provided by exact matching. Regular expressions are an efficient tool to extend exact matching with flexible patterns, that may find different matches. As an example, we may be interested in finding all the mentions of the acronym MHS or MHN in a text. For doing that, regular expressions provide the alternation operator that helps us to solve this issue easily by specifying multiple alternatives to match in a specific part of the pattern, in this case an $S$ or an $N$ as the last character.

Regular expressions can be better understood by clearly separating three distinct components:

input - any string where we want to find something

pattern - a string that specifies what we are looking for

match - a fragment of the input (a substring)

where the pattern can be found

In our examples, the input is the text file chebi_27732.txt, but it can be the amino acid sequences that we previously extracted from the UniProt file entries. Until now the pattern has represented an exact string to look for, where each match is an exact replica of the pattern occurring at a given position of the input string. When using regular expressions, the pattern contains special characters, whose purpose are not to directly match with the input but instead have a special meaning. These special characters represent operators that specify which different types of strings we want to find in the input. For example, strings that start with $M H$ and end with $S$ or an $N$. By using regular expressions, the matches are not replicas of the pattern, they can be different strings as long as they satisfy the specified pattern.

\section{Extended Syntax}

The grep command allows us the possibility to include regular expression operators in the input pattern. grep understands two different versions of regular expression syntax: basic and extended $^{3}$. We will use the extended syntax for two reasons: (i) the basic does not support relevant operators, such as alternation; (ii) and to clearly differentiate exact matching from regular expression matching. Thus, instead of the -e option previously used in the grep command, we will start to use the $-\mathrm{E}$ option, which makes the command interpret the pattern as an extended regular expression. The equivalent long form to the $-\mathrm{E}$ option is --extended-regexp. We should note that this option does not affects the matching when using a pattern without any regular expression operator, such as MH. For example, the following commands will produce the same results:

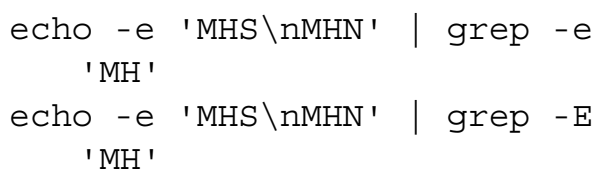

Note, that we use the -e option so the echo command interpret the $\backslash \mathrm{n}$ characters as a newline. Thus, the echo command outputs two lines, that are given as input to the grep command. We should note that the grep command filters lines.

\footnotetext{
${ }^{3}$ https://www.regular-expressions.info/posix.html
} 


\section{Alternation}

The first regular expression operator we will test is the alternation, which we introduced above. An alternation is represented by the bar character (|) that specifies a pattern where any match must include either the preceding or following characters. The preceding and following characters can be enclosed within parentheses to better specify the scope of the alternation operator. For example, the pattern for finding strings that start with $M H$ and end with $S$ or an $N$ can be written as:

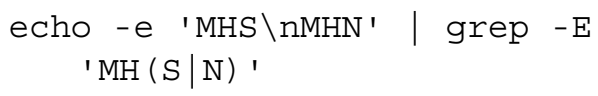

\section{Basic Syntax}

If we use the basic regular expression syntax no match will be found, since the alternation operator is not supported:

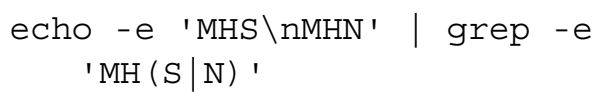

We will have a match only if the $\mid$ and the parentheses are in the input string, since it is not interpreted as an operator:

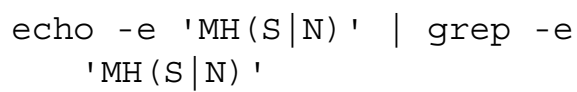

\section{Scope}

To better understand the scope of an alternation, we can remove the parentheses from the pattern and add the - w option:

$$
\begin{gathered}
\text { echo -e 'MHS } \backslash \text { nMHN' | grep -w } \\
\text { - E 'MHS } \mid \text { N' }
\end{gathered}
$$

We only get the first line. This is explained because the alternation operator is applied to all the preceding characters, i.e. the grep will search for the $M H S$ word or the $N$ word. If we add a single $N$ to the input string we already get another match:

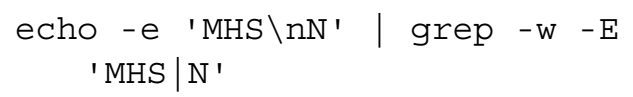

We can also move the opening parenthesis one character to the left:

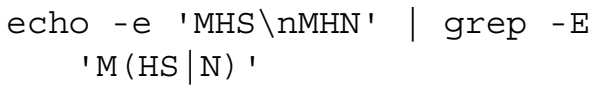

Only $M H S$ is now displayed, since the alternative now represents $M N$ without the $H$.

\section{Multiple Alternatives}

We are not limited to two alternatives, we can have multiple | operators in a pattern. For example, the following command will find any of the three acronyms $M H S, M H E$ or $M H N$ :

$$
\begin{gathered}
\text { echo -e 'MHS } \backslash \mathrm{nMHN} \backslash \mathrm{nMHE} \text { ' } \\
-\mathrm{E} \quad \mathrm{MH}(\mathrm{S}|\mathrm{N}| \mathrm{E}) \text { ' }
\end{gathered}
$$

We can now transform our previous grep command with multiple case sensitive patterns:

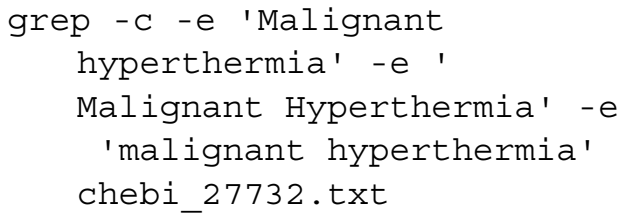

in a grep command with a single pattern using alternation:

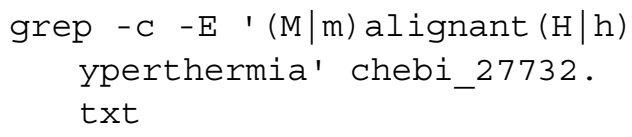

And we will obtain the same 96 matches.

\section{Multiple Characters}

A useful regular expression feature is that we can use the dot character (.) to represent any character, so if we want to find all the acronyms that start with $M H$ we can execute the following command:

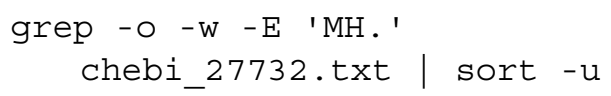

We should note that we use the - o option of the command grep so it just displays the matches and not all the line that includes the match. The equivalent long form to the -o option is - - only - matching.

The output will be the following threecharacter lines: 
$\mathrm{MH}$

$\mathrm{MH})$

$\mathrm{MH}$ ，

$\mathrm{MH}$.

$\mathrm{MH} 1$

MH2

$\mathrm{MHE}$

MHN

MHS

If we really want to match only the dot character, we have to precede it with a backslash character $(\backslash)$ :

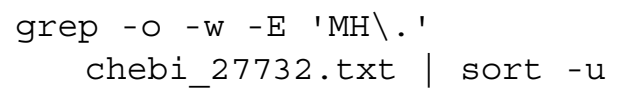

Now only the $M H$. will be displayed.

We can check that there are some matches that are not really acronyms, such as $M H$ ) and $M H$,.

\section{Spaces}

We should note that $M H$ appears because the space character can also be matched. For example, the following text includes a word match with $M H_{\sqcup}$ since the parenthesis is considered a word delimiter character (not a letter, digit or underscore):

... susceptible to $\mathrm{MH}$ (MHS) ...

On the other hand, the following text does not include a word match with $\mathrm{MH}_{\sqcup}$ :

... markers and $\mathrm{MH}$

susceptibility ...

Thus, what we really want is matches where the third character is a letter or a numerical digit.

Sometimes, the text includes other characters that also represent horizontal or vertical space in typography, such as the tab character. All these characters are known as whitespaces and can be represented by the expression $\backslash \mathrm{s}$ in a pattern ${ }^{4}$. The following command demonstrates that both the space and the tab characters are matched by $\backslash \mathrm{s}$ :

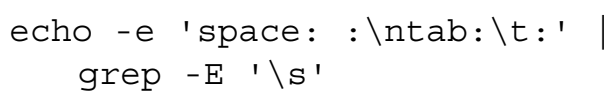

\footnotetext{
${ }^{4}$ https://en.wikipedia.org/wiki/Whitespace_character
}

\section{Groups}

Fortunately, the regular expressions include the group operator that let us easily specify a set of characters. A group operator is represented by a set of characters enclosed within square brackets. Any of the enclosed characters can be matched.

For example, the previous command to find any of the three acronyms can be replaced by:

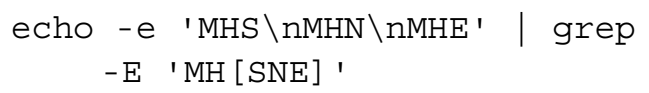

We should note that only one of the three letters, $S, N$ or $E$ will be matched in the input string.

\section{Ranges}

Still, this is not solving our need to only match letters or digit. However, we can also specify characters ranges with the dash character (-). For example, to find all the acronyms that start with $M H$ followed by any alphabet letter:

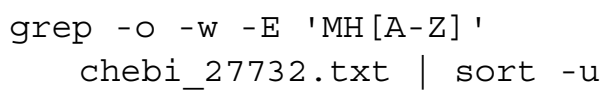

This will result in only three acronyms:

MHE

MHN

MHS

We should note that $\mathrm{A}-\mathrm{Z}$ represents any alphabet letter in uppercase, a lowercase letter will not be matched:

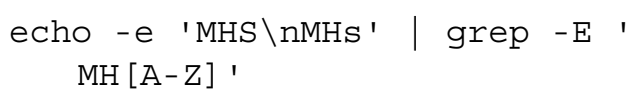

If we intend to keep the usage of a case sensitive grep and at the same time find lowercase matches, then we need to add the $a-z$ range:

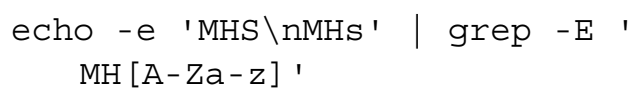

We should note that the dot character inside a range represents itself and not any character:

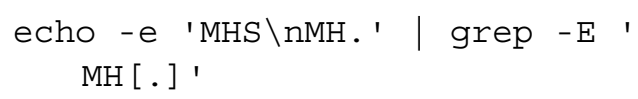

Additionally, to include the acronyms that end with a numerical digit we need to add the 0-9 range: 


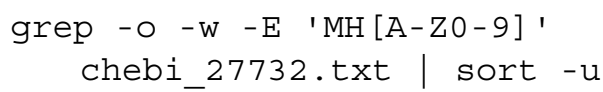

Finally, we have the correct list of all three character acronyms starting with $M H$ :

MH1

$\mathrm{MH} 2$

$\mathrm{MHE}$

MHN

MHS

\section{Negation}

Another frequent case is the need to match any character with a few exceptions. For example, if we need to find all the matches that start with $M H$ followed by any character except an alphabet letter. Fortunately, we can use the negation feature within a group operator. The negation feature is represented by the circumflex character $\left(^{\wedge}\right)$ right next to the left bracket. The negation means that all the characters and ranges enclosed within the brackets are the ones that cannot be matched. Thus, a solution to the above example is to add the $\mathrm{A}-\mathrm{Z}$ range after the circumflex:

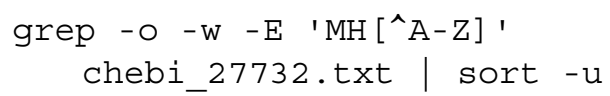

We can see that all of the three acronyms $M H S, M H E$ or $M H N$ will be missing from the output:

$\mathrm{MH}$

$\mathrm{MH}$ ，

$\mathrm{MH}$.

$\mathrm{MH}$ )

MH1

MH2

If we do not want the $\mathrm{MH}_{\sqcup}$ acronym, we can add the space character to the negative group:

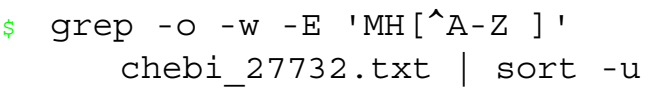

The output should now contain one less acronym:

$\mathrm{MH}$,

$\mathrm{MH}$.

\section{Quantifiers}

Above we were interested in finding acronyms composed of exactly three characters. However, we may need to find all acronyms that start with $M H$ independently of their length. This functionality is also available in regular expressions using the quantifiers operators.

\section{Optional}

The simplest quantifier is the optional operator that is specified by an item followed by the question mark character (?). The item can be a character, an operator or a sub-pattern enclosed by parentheses. That item becomes optional for matching, i.e. a match can either contain that item or not.

For example, to find all the acronyms starting with $M H$ and followed by one alphabetic letter or none:

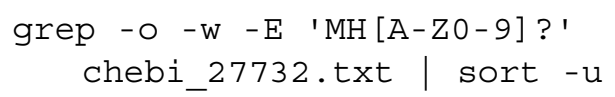

Given that the third character is optional the output will include the two-character acronym $\mathrm{MH}$, but not the $\mathrm{MH}_{\sqcup}$ match:

$\mathrm{MH}$

MH1

$\mathrm{MH} 2$

MHE

MHN

MHS

We can add the space character to the group:

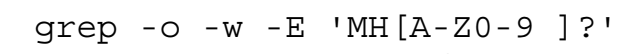

Now the output includes the two-character acronym $\mathrm{MH}$ and the $\mathrm{MH}_{\sqcup}$ match:

$\mathrm{MH}$

$\mathrm{MH}$

MH1

$\mathrm{MH} 2$

MHE

MHN

MHS 


\section{Multiple and Optional}

To find all the acronyms independently of their length, we can use the asterisk character $(*)$. The preceding item becomes optional and can be repeated multiple times. For example, to find all the acronyms starting with $M H$ and which may be followed any number of alphabetic letters or numeric digits:

$$
\begin{aligned}
& \text { grep -o - w }-\mathrm{E} \quad \text { 'MH [A-Z0-9]*' } \\
& \text { chebi_27732.txt | sort -u }
\end{aligned}
$$

The output now includes the four-character acronym MHS1:

$\mathrm{MH}$

$\mathrm{MH} 1$

MH2

MHE

MHN

MHS

MHS 1

We should note that the grep command uses a greedy approach, i.e. it will try to match as many characters as possible. For example, the following command will match $\mathrm{MHI}$ and not $M H$ :

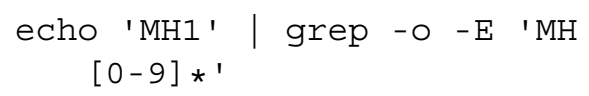

\section{Multiple and Compulsory}

To make the preceding item compulsory and able to repeat it multiple times, we may replace the asterisk by the plus character (+). For example, the following pattern will find all the acronyms starting with $M H$ followed by at least one alphabetic letter or numeric digit:

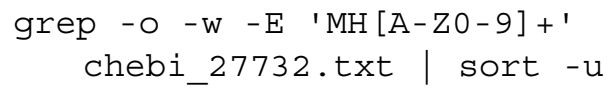

We should note that the output does not contain the two character acronym $M H$ :

$\mathrm{MH} 1$

MH2

MHE

MHN

MHS

MHS1

\section{All Options}

The above quantifiers are the most popular, but the functionality of all of them can be reproduced by using curly braces to specify the minimal and maximum number of occurrences. The item is followed by an expression of the type $\{n, m\}$ where $n$ and $m$ are to be replaced by a number specifying the minimum and maximum number of occurrences, respectively. $\mathrm{n}$ and $\mathrm{m}$ may also be omitted, which means that no minimum or maximum limit is to be imposed.

Using curly brackets, the question mark character (?) can be replaced by $\{0,1\}$. Thus, the following two patterns are equivalent:

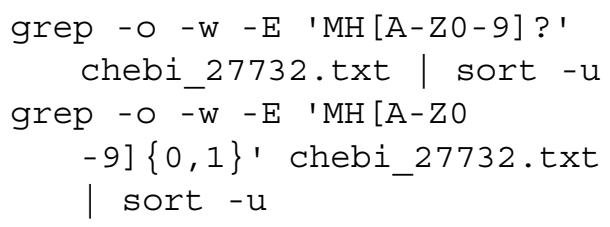

The asterisk character $(*)$ can be replaced by $\{0$,$\} . Thus, the following two patterns are$ equivalent:

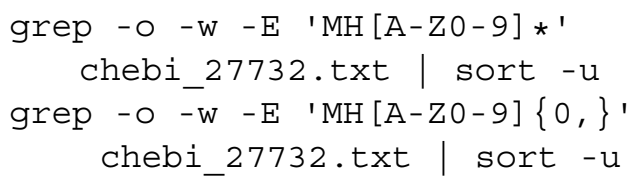

The plus character $(+)$ can be replaced by $\{1$,$\} . Thus, the following two patterns are$ equivalent:

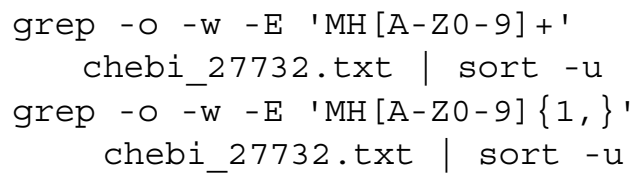

On the other hand using $\{1,1\}$ is the same as not having any operator. Thus, the following two patterns are equivalent:

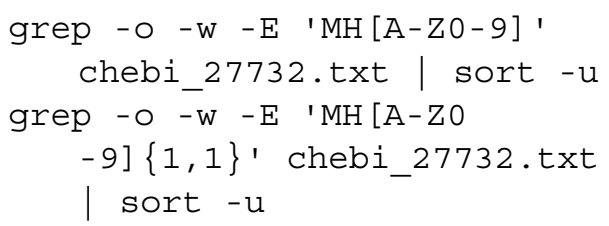

The previous commands display the all the three-character acronyms: 
$\mathrm{MH} 1$

$\mathrm{MH} 2$

MHE

MHN

MHS

For example, if we are looking for acronyms with exactly 4 characters then we can apply the following pattern:

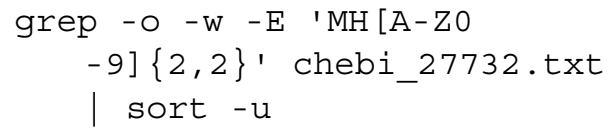

We should note that we use 2 as both the minimum and maximum since $M H$ already count as 2 characters.

The output of the previous command is now the four-character acronym:

MHS 1

\section{Position}

Sometimes besides the match, we are also interested in limiting the matches to specific parts of the input string. For example, to identify start and stop codons in a protein sequence, we need to limit the matches to the beginning or the end of the sequence. In text, we may for example be interested in lines starting with a name of a disease. To take in account the position of a match regular expressions patterns can start with the circumflex character $\left({ }^{\wedge}\right)$ and/or end with the dollar sign character $(\$)$.

If the pattern starts with a circumflex then only matches at the beginning of the line will be considered. On the other hand, if the pattern ends with a dollar then only matches at the end of the line will be considered.

\section{Beginning}

For example, if we are looking for lines starting with Malignant Hyperthermia we can use the following pattern:

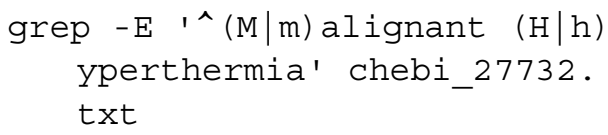

The output will include the list of lines beginning with a mention to Malignant Hyperthermia:

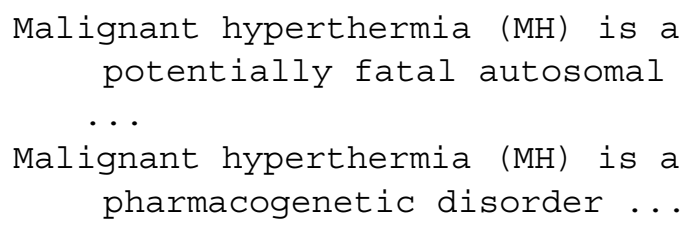

Malignant hyperthermia (MH) is a pharmacogenetic disorder ...

To check how many of the matching lines were filtered, we can count the number of occurrences when using the circumflex and when not:

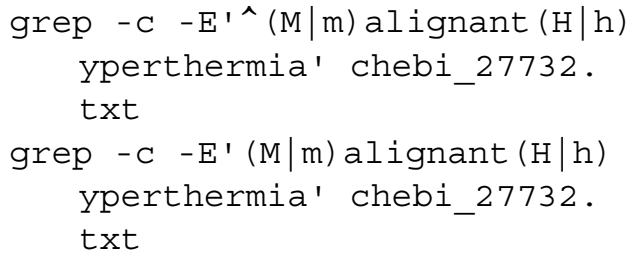

The output will show that only 23 of the 96 matches were considered.

\section{Ending}

If we are looking for lines ending with a mention to Malignant Hyperthermia, then we can add the dollar character to the end of the pattern:

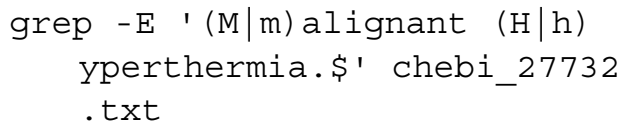

To allow a punctuation character before the end of the line, we added the dot character before the dollar character in the pattern. The dot character matches any character, including the dot itself.

The output will be the list of lines ending with a mention to Malignant Hyperthermia:

Novel mutation in the RYR1 gene (R2454C) in a patient with malignant hyperthermia. 


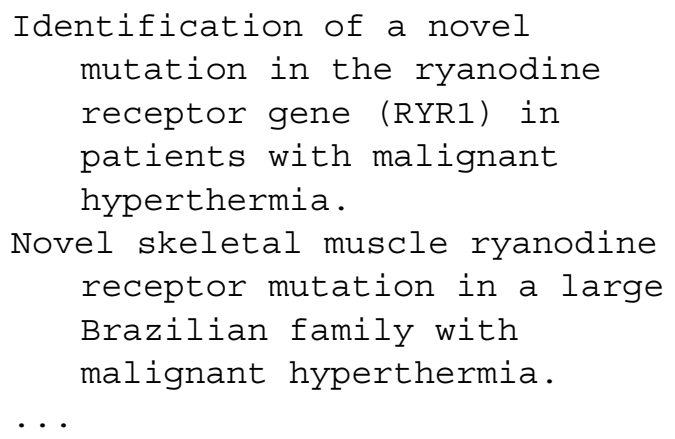

We can check how many lines were filtered by using again the $-\mathrm{C}$ option:

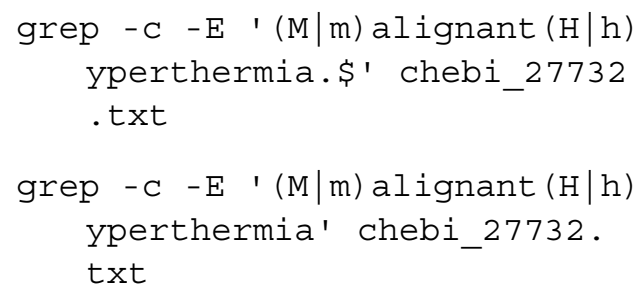

The output will show that only 15 of the 96 matches were at the end of the line.

\section{Near the End}

Sometimes we do not want the mention ending exactly at the last character. We may be more flexible and allow a following expression, or a given number of characters. For example, to allow 10 other characters between the end of the line and the mention of Malignant Hyperthermia, we can add a quantifier to the dot operator:

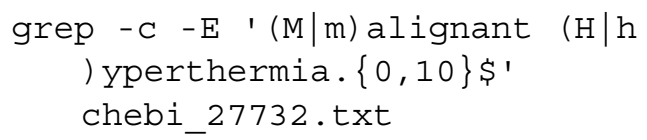

The output will show that we have 20 matches.

If we remove the $-c$ option, we will be able to check that words, such as families and patients, are now allowed to appear between the mention of Malignant Hyperthermia and the end of the line:

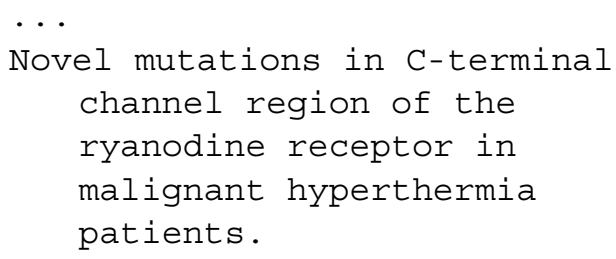

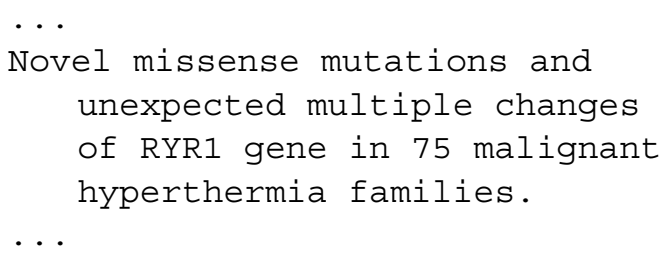

\section{Word in Between}

To allow a word in between, independently of its length, we can add to the pattern an optional sequence of non-space characters (the word) preceded by a space:

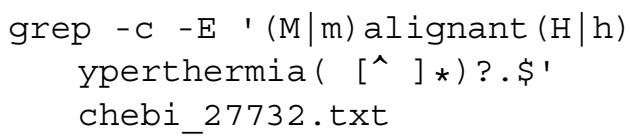

The output will show that we have 24 matches. We should note that the $\left[{ }^{\wedge}\right]$ operator avoids having two words.

If we remove the - $\mathrm{c}$ option, we will be able to check that lengthy words (with more than 10 characters), such as susceptibility, are now allowed to appear between the mention of $M a$ lignant Hyperthermia and the end of the line:

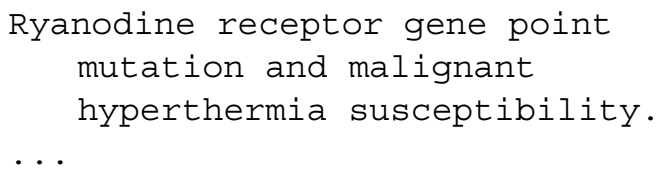

\section{Full Line}

If we want lines that start with a mention to $M a$ lignant Hyperthermia and end with an acronym, $M H$ or $M H S$, then we can execute two grep commands. The first gets the lines starting with Malignant Hyperthermia and the next filters the output of the latter with lines ending with an acronym:

$$
\begin{gathered}
\text { grep }-\mathrm{E} \text { '^ }(\mathrm{M} \mid \mathrm{m}) \text { alignant }(\mathrm{H} \mid \mathrm{h}) \\
\text { yperthermia' chebi_27732. } \\
\text { txt | grep -w -E 'MHS?.\$' }
\end{gathered}
$$

Alternatively, we can add both the circumflex and dollar operators to the same pattern. However, we cannot forget to add .* to match 
anything in between them, since we are asking full line matches:

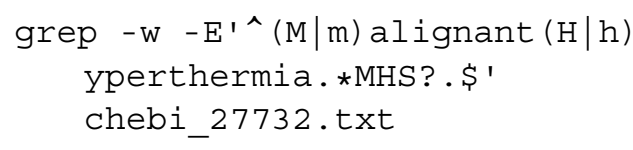

We can see that both commands match all the text of the abstract since each abstract is stored in a single line of the file:

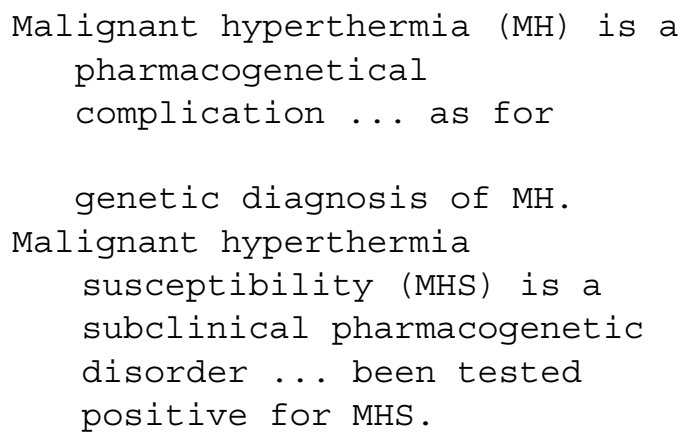

This demonstrates the problem of tokenization, since usually what we really need is to match a full sentence or a phrase. And in that case each line should represent a sentence or phrase from the abstract.

\section{Match Position}

For more advanced processing, we may be interested in knowing the exact position of the matches in a given line. This can be done by using the - b option of grep, which provides the number of bytes in the line before the start of the match:

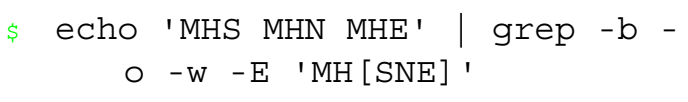

The equivalent long form to the - b option is - byte-offset.

The output shows the list of matches preceded by their position in the given line:

\section{$0:$ MHS \\ $4:$ MHN \\ $8:$ MHE}

\section{Tokenization}

As we have shown in the previous section, sometimes we need to work at the level of a sentence and not use a full document as the input string. Tokenization is a Natural Language Processing (NLP) task that aims at identifying boundaries in the text to fragment it into basic units called tokens. These tokens can be sentences, phrases, multi-word expressions, or words.

\section{Character Delimiters}

In most languages, some specific characters can be considered as accurate boundaries to fragment text into tokens. For example, the space character to identify words; the period (.), the question mark (?) and the exclamation mark (!) to identify the ending of a sentence; and the comma (,), the semicolon (;), the colon (:) or any kind of parenthesis to identify a phrase within a sentence. However, this problem may be more complex in languages without explicitly delimiters, such as Chinese (Wu and Fung 1994).

A common approach to tokenization is to use regular expressions to replace these delimiters by newline characters. This will result in a token per line. For example, we can replace the characters specifying the end of a sentence with a newline by using the tr command and then count the number of lines:

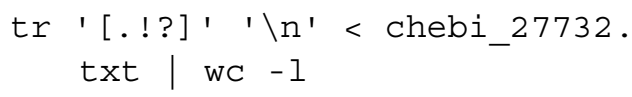

We get 1493 lines from the original 248 lines:

wC -1 chebi_27732.txt

Unfortunately, this is not just so simple. We need to analyze the output:

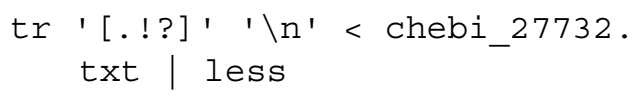




\section{Wrong Tokens}

We can check that: (i) many lines are empty because an extra newline character will be added to the last sentence, and (ii) the dot character is also used as a decimal mark in a number, then some sentences are split in multiple lines because they have decimal number in them. For example, the original sentence:

\section{These 10 mutations account for \\ $21.9 \%$ of the North American $\mathrm{MH}$-susceptible population}

is split in two lines:

\section{These 10 mutations account for 21 \\ 9\% of the North American MH- susceptible population}

\section{String Replacement}

This means that looking at just one character is not enough, we need some context. For performing this, we will use the sed command that we may consider as a more powerful version of the tr command. The sed command is a stream editor that can receive as input a string and perform basic text transformations, such as replace one expression by another, that are available in almost all text editors. For example, we can use a simple sed to convert every mention of caffeine by its ChEBI identifier:

$$
\begin{aligned}
& \text { sed }-E \text { 's/caffeine/CHEBI } \\
& \text { :27732/gi' chebi_27732.txt }
\end{aligned}
$$

The $-\mathrm{E}$ option allow us to use extended regular expressions, like we used before in grep. The $\mathrm{s}$ option has the following syntax 's/FIND/ REPLACE/FLAGS ', where: FIND is the pattern to find in the input string; REPLACE the expression to replace the matches; FLAGS are multiple options, such as $g$ to replace all matches in each line and not just the first one, and $i$ to be case insensitive.
For example, the original fragment of text:

$$
\begin{gathered}
\text {... link between the caffeine } \\
\text { threshold and tension ... }
\end{gathered}
$$

will be converted to:

\section{... link between the CHEBI:27732 \\ threshold and tension ...}

\section{Multi-character Delimiters}

To replace the delimiter characters by a newline when followed by at least one space character, we can use the following command:

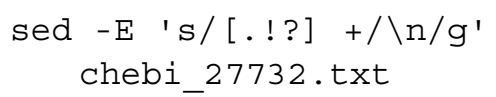

We should note that by making compulsory a space character, we avoid: (i) empty lines by splitting a sentence that is already at the end of the line (assuming there are no ghost space characters at the end of each line), and (ii) decimal markers because they are followed by numerical digits and not spaces.

We now get 1067 lines from the original 248 lines:

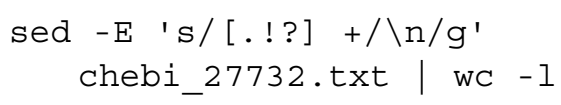

\section{Keep Delimiters}

The previous sed command is removing the delimiter characters from the text, and this may cause other problems. The best solution is to keep the delimiter characters and just add the newline. The sed command allows us to keep each match for a specific part of the pattern (sub-pattern) by enclosing it within parentheses. To include the match of a subpattern in the replace expression, we can use the backslash and its numerical order. Thus, we can improve our sed command by using this technique so we do not remove any delimiter character:

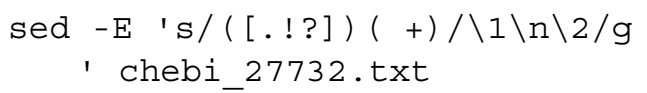


However, other common issues may still persist. For instance, there are some sentences starting right after the delimiter characters without any space in between:

$$
\begin{aligned}
& \ldots \text { bulk. Fetal ... } \\
& \ldots \text { sequencing. Whole ... }
\end{aligned}
$$

These sentences include a delimiter character directly followed by an alphabetic letter:

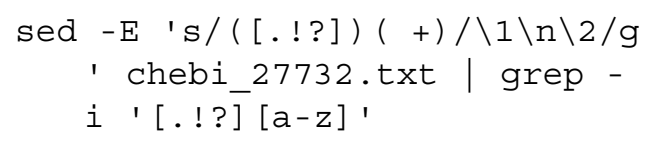

To minimize this issue, we can change the pattern so the compulsory space character become optional, but requiring a following uppercase alphabetic letter:

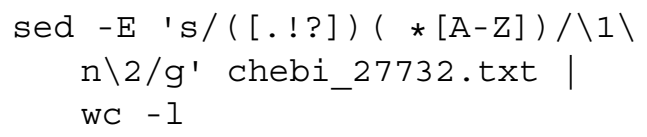

We now get 1127 lines, i.e. this pattern is more flexible and was able to split more 60 sentences. This does not mean that is free of errors. It is almost impossible to derive a rule that covers all the possible typos humans can produce.

As an example, Fig. 4.1 show a complex pattern adapted from Wikipedia. The pattern is equivalent to $\backslash .\{2\},[\mathrm{A}-\mathrm{Z}]$, and identifies multiples spaces at the beginning of a sentence. The pattern requires at least two spaces to be matched, but only after a period and before an uppercase letter.

\section{Sentences File}

Using our previous pattern, we can update our script named gettext.sh to provide the text already split in sentences by adding the sed command:

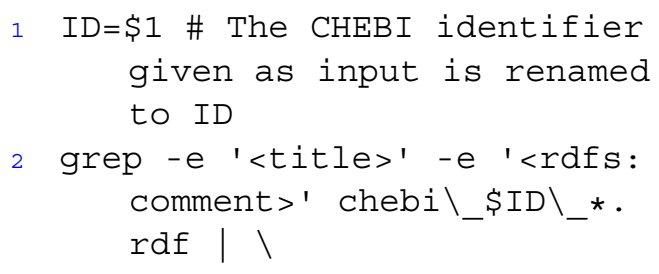

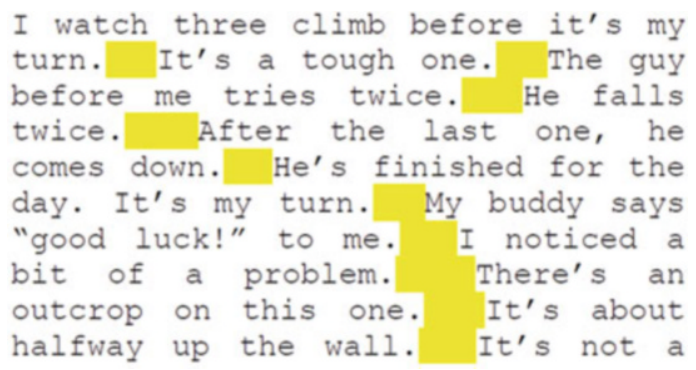

Fig. 4.1 Identifying multiple spaces at the beginning of a sentence using regular expressions (Adapted from: https:// en.wikipedia.org/wiki/Regular_expression)

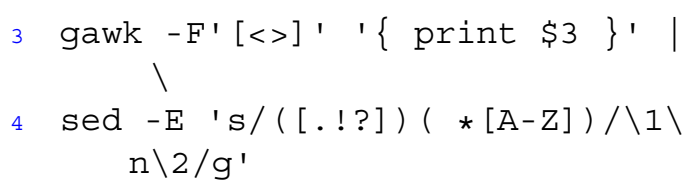

To save the output as a file named chebi_27732_ sentences.txt, we only need to add the redirection operator:

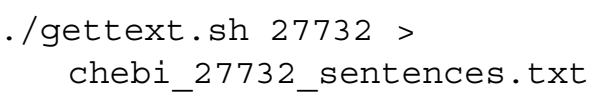

Each line of the file chebi_27732_sentences.txt represents a sentence.

\section{Entity Recognition}

To select the sentences with one of our acronyms, we can use the grep command and our sentences file:

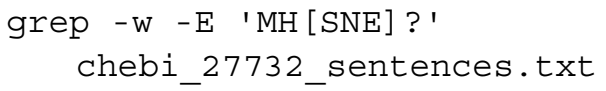

The output will only include matching sentences:

Interestingly, the data suggest
a link between the caffeine threshold and tension values and the MH/CCD phenotype.

Alternatively, we can use the $-\mathrm{n}$ option to get the number of the line and the -o option to get the acronym matched: 


\section{grep - $\mathrm{n}-\mathrm{O}-\mathrm{w}-\mathrm{E}$ 'MH [SNE] ?' chebi_27732_sentences.txt}

The equivalent long form to the $-\mathrm{n}$ option is - - line-number. The output should be something like this:

...

$1106: \mathrm{MH}$

$1106: \mathrm{MH}$

$1108: \mathrm{MH}$

$1110: \mathrm{MH}$

$1111: \mathrm{MH}$

We can now make a script that receives a pattern as argument and the input text as the standard input, to display the line numbers and the matches in a TSV format. Thus, let us create a script file named getentities.sh with the following lines:

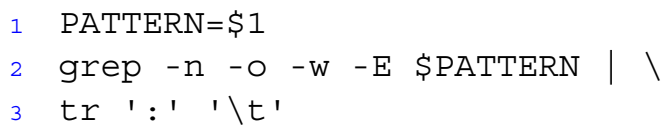

Again we should not forget to save the file in our working directory, and add the right permissions with chmod, as we did with our scripts in the previous chapter.

The first line stores the pattern given as argument in the variable PATTERN. The grep command finds the matches and the tr command replaces each colon by a tab character to produce TSV content.

We can now execute the script giving the pattern as argument and the sentences file as standard input:

\section{. /getentities.sh 'MH [SNE] ?' < chebi_27732_sentences.txt}

The output should be something like this:

$\begin{array}{ll}\cdots & \\ 1106 & \mathrm{MH} \\ 1106 & \mathrm{MH} \\ 1108 & \mathrm{MH} \\ 1110 & \mathrm{MH} \\ 1111 & \mathrm{MH}\end{array}$

We should note that now we have the values separated by a tab character, i.e. the output is in TSV format.
The output can also be saved as a TSV file that we can open directly in our preferred spreadsheet application. For example, to save it as chebi_27732.tsv, we only need to add the redirection operator:

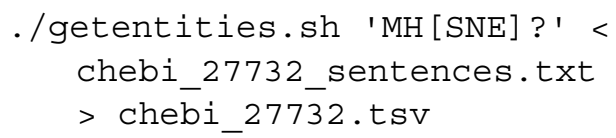

\section{Select the Sentence}

If we want to analyze a specific matched sentence, we can use a text editor and go to that line number. A more efficient alternative is to use the print $\mathrm{p}$ option of sed to output a given line number. For example, to check the $M H S$ match at line 2:

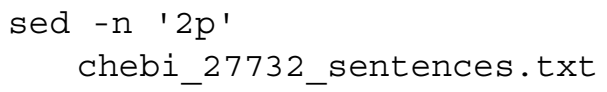

Now we can easily check the context of the match:

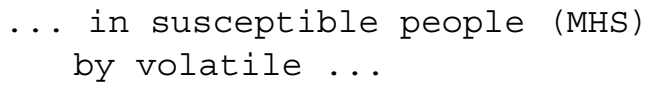

\section{Pattern File}

The script created in the previous section only accepts one pattern, however we may need to recognize different entities, or different mentions of the same entity, such as the official name, possible synonyms, and the acronyms. Fortunately, grep allows us to include a list of patterns directly from a file using the $-f$ option. The equivalent long form to the $-\mathrm{f}$ option is - file=FILE. For example, we can create a text file named patterns.txt with the following three patterns:

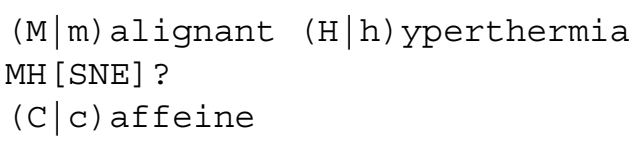

Then we can execute the previous grep but using multiple patterns specified in the pattern file: 


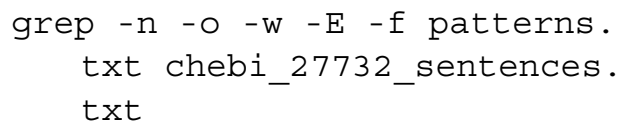

Analyzing the output, we can check that the same sentences may include different entities: ...

$1110: \mathrm{MH}$

1110 : caffeine

1111 : caffeine

$1111: \mathrm{MH}$

We can now update our script named getentities.sh to receive as input not a single pattern but the filename where multiple patterns can be found.

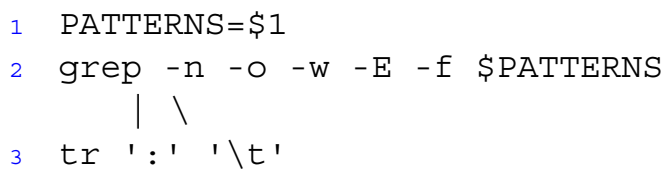

We can execute the script giving as argument the file containing the patterns:

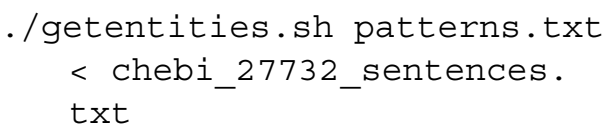

To save the output as a file named chebi_27732.tsı,111: . . caffeine threshold and we only need to add the redirection operator:

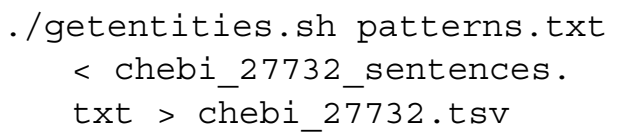

Using the patterns.txt file is very useful if for example we are not focused in a single disease, and we want to find any disease mentioned in the text. In these cases, we have to create a file with the full lexicon of diseases. This topic will be addressed in the following chapter.

\section{Relation Extraction}

Finding the relevant entities in text is sometimes not enough. We need to know which sentences may describe possible relationships between those entities, such as a relation between a disease and a compound.
This is a complex text mining challenge, but a simple approach is to construct a pattern that allow any kind of characters between two entities:

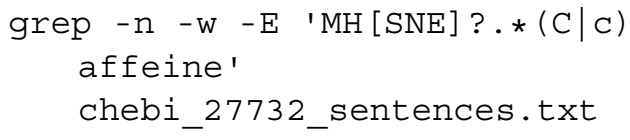

The following sentence is one of the seven displayed sentences mentioning a possible relation:

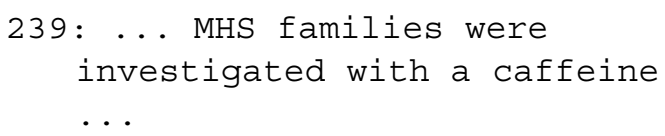

However, we are missing all the sentences that have caffeine first:

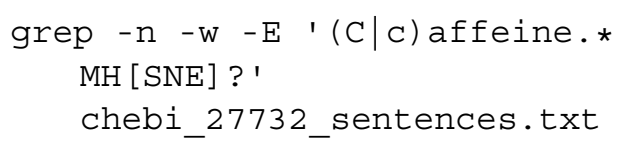

We will be able to see that sometimes caffeine comes first:

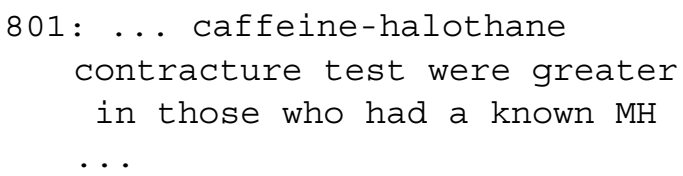

\section{Multiple Filters}

The most flexible approach is use two grep commands. The first selects the sentences mentioning one of the entities, and the other selects from the previously selected sentences the ones mentioning the other entity. For example, we can first search for the acronyms and then for caffeine:

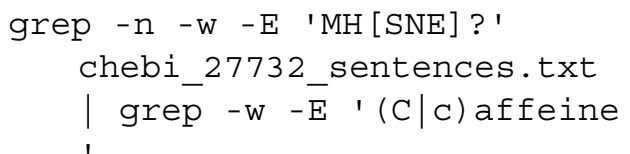

This will show all the nine sentences mentioning caffeine and an acronym. 


\section{Relation Type}

If we are interested in a specific type of relationship, we may have an additional filter for a specific verb. For example, we can add a filter for sentences with the verb response or diagnosed:

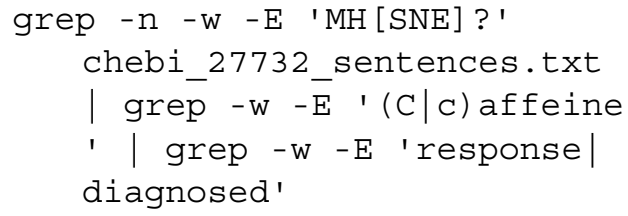

We should note that this does not take in account where the verb appears in the sentence. For example, in the following sentence the verb response appears first than any of the two entities:

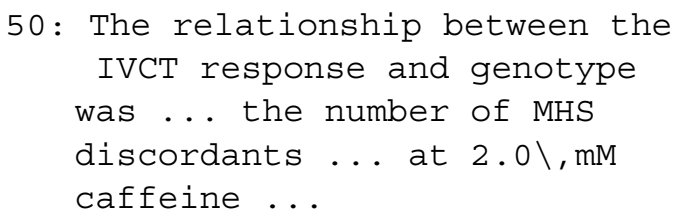

If the verb needs to appear between the two entities, we have to construct a pattern that have these words in the middle of them:

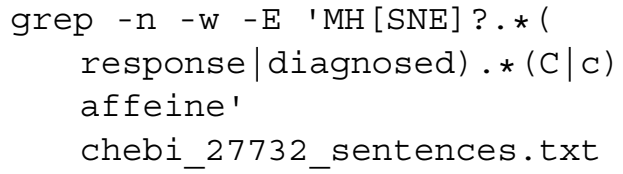

We can see now that the previous sentence (line 50) is not presented as a match.

\section{Remove Relation Types}

We may also be interested in ignoring specific type of relations. To do that, we only need to use the -v (or - -invert-match) option. For example, to ignore sentences with the word response or diagnosed:

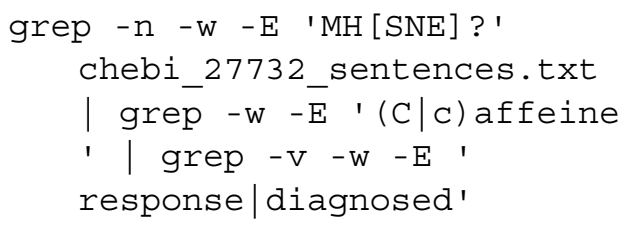

All the resulting sentences do not mention response or diagnosed.

\section{Further Reading}

If we want to have a deeper knowledge about text processing tasks and challenges, we may be interested in reading some chapters of the book entitled Speech and language processing (Jurafsky and Martin 2014). The book is a highly specialized document explaining in full detail the topics here briefly described.

To have an overview about the state-of-art in text processing tools using biomedical literature, we should consider reading a recent and comprehensive survey (Lamurias and Couto 2019).

Open Access This chapter is licensed under the terms of the Creative Commons Attribution 4.0 International License (http://creativecommons.org/licenses/by/4.0/), which permits use, sharing, adaptation, distribution and reproduction in any medium or format, as long as you give appropriate credit to the original author(s) and the source, provide a link to the Creative Commons licence and indicate if changes were made.

The images or other third party material in this chapter are included in the chapter's Creative Commons licence, unless indicated otherwise in a credit line to the material. If material is not included in the chapter's Creative Commons licence and your intended use is not permitted by statutory regulation or exceeds the permitted use, you will need to obtain permission directly from the copyright holder. 


\section{Semantic Processing}

\section{Abstract}

In the previous chapter we were able to automatically process text by recognizing a limited set of entities. This chapter will introduce the world of semantics, and present step-by-step examples to retrieve and enhance text and data processing by using semantics. The goal is to equip the reader with the basic set of skills to explore semantic resources that are nowadays available using simple shell script commands.

\section{Keywords}

Ontologies · OWL: Web Ontology

Language - Semantic resources - DO: disease ontology - ChEBI: chemical entities of biological interest - Ancestors - Recursion . Lexicons $\cdot$ Entity linking $\cdot$ Semantic similarity

\section{Classes}

In the previous chapters we searched for mentions of caffeine and malignant hyperthermia in text. However, we may miss related entities that may also be of our interest. These related entities can be found in semantic resources, such as ontologies. The semantics of caffeine and malignant hyperthermia are represented in ChEBI and DO ontologies, respectively.

\section{OWL Files}

Thus, we can start by retrieving both ontologies, i.e. their OWL files.

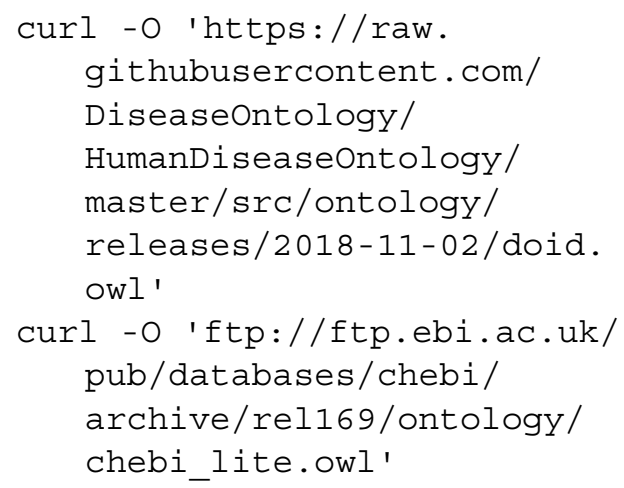

The $-O$ option saves the content to a local file named according to the name of the remote file, usually the last part of the URL. The equivalent long form to the -O option is - - remote-name.

The previous commands will create the files chebi_lite.owl and doid.owl, respectively. We should note that these links are for the specific releases used in this book. Using another release may change the output of the examples presented in this chapter.

The links may also change in the future, so we may need to check them on the BioPortal ${ }^{1}$ or

${ }^{1}$ http://bioportal.bioontology.org/ 
on the OBO Foundry ${ }^{2}$ webpages. Alternatively, we can also get the OWL files from the book file archive $^{3}$.

\section{Class Label}

Both OWL files use the XML format syntax. Thus, to check if our entities are represented in the ontology, we can search for ontology elements that contain them using a simple grep command:

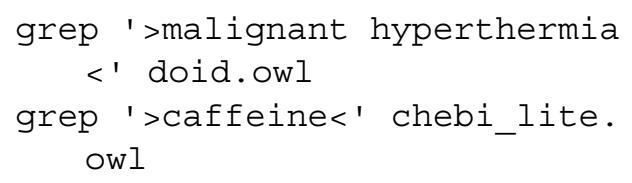

For each grep the output will be the line that describes the property label ( $r d f s:$ label), which is inside the definition of the class that represents the entity:

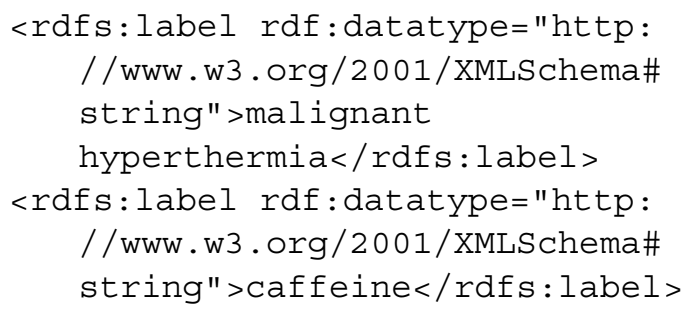

\section{Class Definition}

To retrieve the full class definition, a more efficient approach is to use the xmllint command, which we already used in previous chapters:

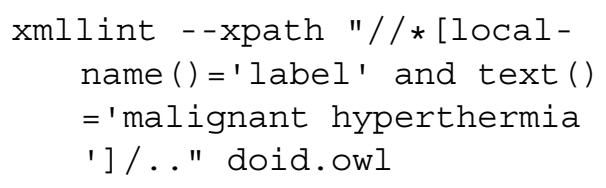

The XPath query starts by finding the label that contains malignant hyperthermia and then .. gives the parent element, in this case the Class element.

\footnotetext{
${ }^{2}$ http://www.obofoundry.org/

${ }^{3}$ http://labs.rd.ciencias.ulisboa.pt/book/
}

From the output we can see that the semantics of malignant hyperthermia is much more than its label:

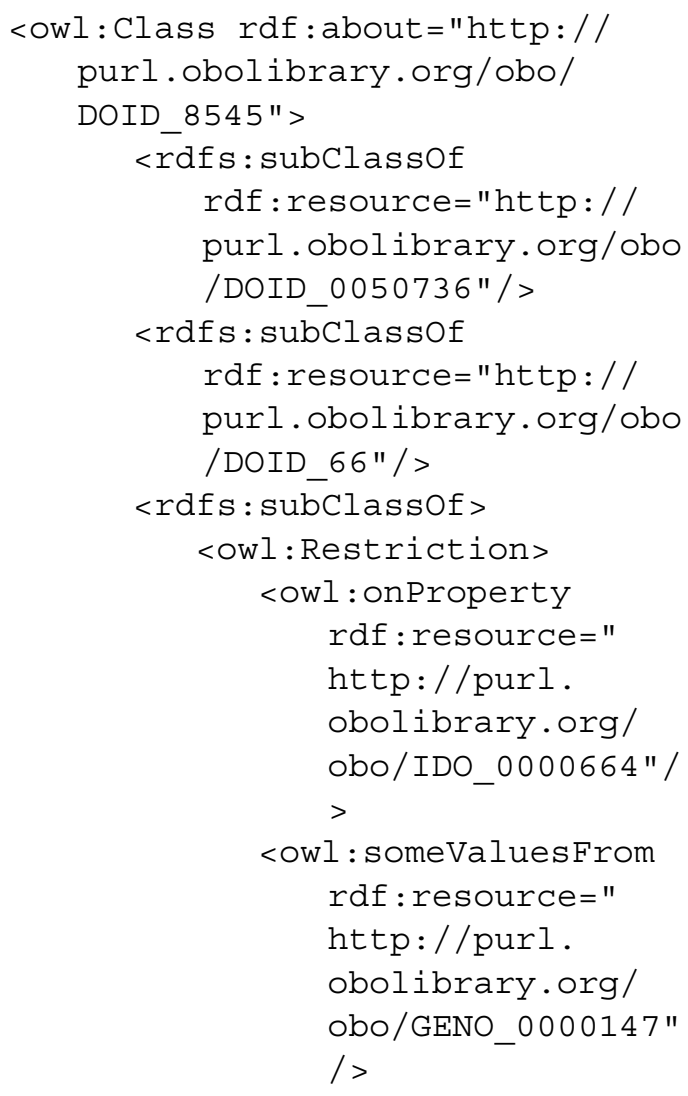


www . w3 . org/2001/

XMLSchema\#string" >

malignant hyperpyrexia

due to anesthesia</

oboInOwl : hasExact Synonym $>$

<oboInOwl : hasOBONamespace

rdf : datatype="http : / /

www.w3.org/2001/

XMLSchema\#string" >

disease_ontology</

oboInOwl : hasOBONamespace $>$

<oboInOwl:id rdf:datatype=

"http: / / www.w3 .org

/2001/XMLSchema\#string"

>DOID : 8545 </oboInOwl : id

$>$

<oboInOwl : insubset

rdf : resource = "http: / /

purl.obolibrary.org/obo

/doid\#DO_MGI_slim"/>

<oboInOwl : insubset

rdf : resource $=$ "http : / / purl.obolibrary.org/obo /doid\#DO_rare_slim"/> <oboInOwl: insubset

rdf : resource $=$ "http $: / /$

purl.obolibrary.org/obo /doid\#NCIthesaurus" / > $<$ rdfs:comment rdf:datatype $="$ http $: / /$ www $\cdot \mathrm{w} 3$.org

/2001/XMLSchema\#string" >Xref MGI.

OMIM mapping confirmed by DO. [ $\mathrm{SN}] .</$ rdfs : comment $>$

$<$ rdfs:label rdf: datatype="

http: //www.w3.org/2001/

XMLSchema\#string" >

malignant hyperthermia<

/rdfs:label>

$<$ owl:Class $>$

A graphical visualization of this class is depicted in Fig. 5.1.

For example, we can check that malignant hyperthermia is a subclass of (specialization) the entries 0050736 and 66 . We can directly use the

\section{Class: malignant hyperthermia}

Term IRI: http:/lpurl.obolibrary.org/obo/DOID 8545

Definition: A muscle tissue disease that is characterized by a drastic and uncontrolled increase in skeletal muscle oxidative metabolism, which overwhelms the body's capacity to supply oxygen, remove carbon dioxide, and regulate body temperature. [database_cross_reference:

url:http://en.wikipedia.org/wiki/Malignant_hyperthermia][database cross_reference: url:http:/len.wikipedia.org/wiki/Malignant_hyperthermia]

[database cross reference: url:http:l/en.wikipedia org/wiki/Malignant hyperthermia][database cross reference:

url:http://en,wikipedia.org/wiki/Malignant_hyperthermia][database_cross_reference: url:http://en.wikipedia.org/wiki/Malignant_hyperthermia]

\section{Annotations}

- database cross reference: ICD9CM:995.86; MESH:D008305; ICD10CM:T88.3; UMLS CUI:C0024591; ORDO:423; CSP2005:2871-4352; GARD:6964;

MTHICD9_2006:995.86; NCl:C84869; OMIM:PS145600

- has_exact_synonym: anesthesia related hyperthermia; malignant hyperpyrexia due to anesthesia

- has_obo_namespace: disease_ontology

- http:I/WWW.W3.org/2000/01/rdf-schemattcomment: Xref MGI. OMIM mapping confirmed by DO. [SN].

- id: DOID:8545

- in subset: DO MGI slim; DO rare slim; NClthesaurus

\section{Class Hierarchy}

Thing

+ disease

+ disease of anatomical entity

+ musculoskeletal system disease

+ muscular disease

+ muscle tissue disease

- distal arthrogryposis

- rippling muscle disease 2

- rippling muscle disease

- myostatin-related muscle hypertrophy

- myotonia congenita

+ myopathy

- malignant hyperthermia

Fig. 5.1 Class description of malignant hyperthermia in the Human Disease Ontology (Source: http://www.ontobee. org/) 
$\operatorname{link}^{4}$ in our browser to know more about this parent disease. We will see that it represents a muscle tissue disease. This means that malignant hyperthermia is a special case of a muscle tissue disease.

We can do the same to retrieve the full class definition of caffeine:

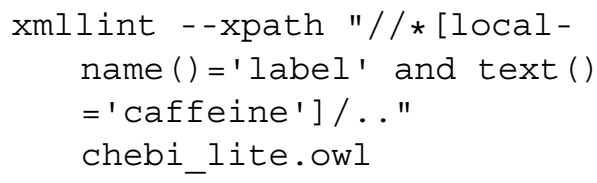

From the output we can see that the types of semantics available for caffeine differs from the semantics of malignant hyperthermia, but they still share many important properties, such as the definition of subclassOf:

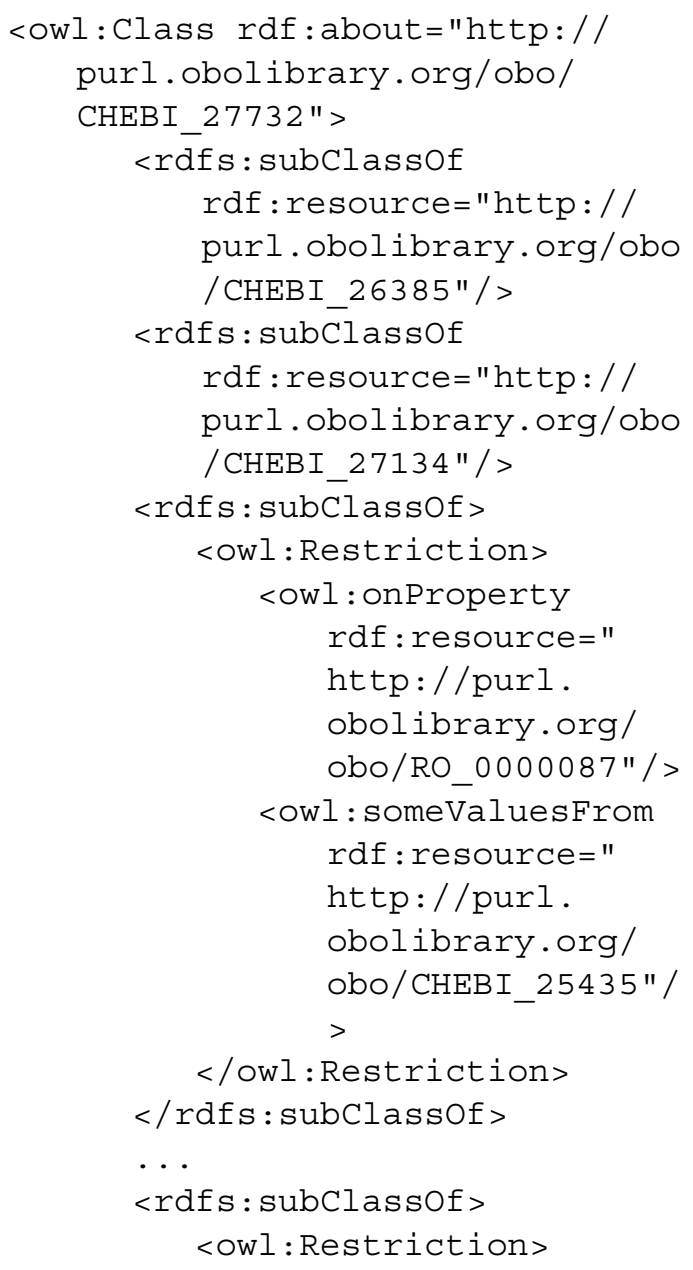

${ }^{4}$ http://purl.obolibrary.org/obo/DOID_66

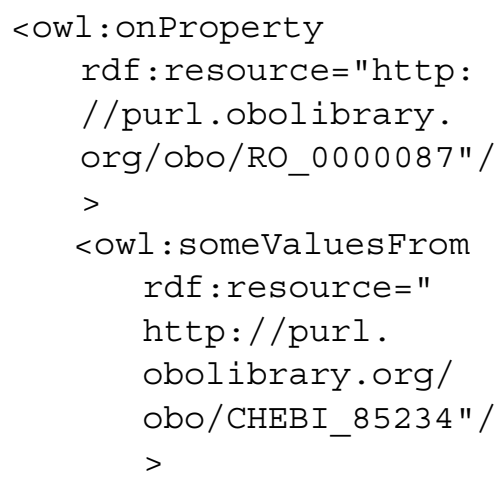

$<$ /owl: Restriction>

$</$ rdfs: subClassOf >

<Obo: IAO_0000115

rdf : datatype="http $: / /$

www.w3.org/2001/

XMLSchema\#string" >A

trimethylxanthine in which the three methyl groups are located at positions 1,3 , and 7 . A purine alkaloid that occurs naturally in tea and coffee. $</$ obo:IAO_0000115> <oboInOwl: hasAlternativeId rdf $:$ datatype $=$ "http $: / /$ www.w3 .org/2001/XML Schema\#string" > CHEBI : 22982 </oboInOwl : has AlternativeId> <oboInOwl : hasAlternativeId rdf $:$ datatype $=$ "http $: / /$ www.w3.org/2001/ XMLSchema\#string" > CHEBI : 32 95</oboInOwl : hasAlternativeId> <oboInOwl : hasAlternativeId rdf : datatype $=$ "http $: / /$ www.w3 .org/2001/XML Schema\#string" >CHEBI : $41472<$ /oboInOwl : hasAlternativeId> <oboInOwl : hasOBONamespace rdf : datatype= "http : / / www.w3.org/2001/ XMLSchema\#string" > 
chebi_ontology $<$ /

oboInOwl : hasOBONamespace $>$

<oboInOwl: id rdf: datatype=

"http: / / www.w3 .org

/2001/XMLSchema\#string"

$>$ CHEBI : $27732<1$

oboInOwl: id>

<oboInOwl : insubset

rdf : resource="http: //

purl.obolibrary.org/obo

/chebi\#3_STAR"/>

<rdfs: label rdf : datatype="

http: //www.w3 .org/2001/

XMLSchema\#string" >

caffeine</rdfs:label>

$<$ owl: Class >

A graphical visualization of this class is depicted in Fig. 5.2.

The class caffeine is a specialization of two other entries: 26385 (purine alkaloid $^{5}$ ), and 27134 (trimethylxanthine ${ }^{6}$ ). However, it contains additional subclass relationships that do not represent subsumption (is-a).

\section{Related Classes}

Figures 5.3 and 5.4 show other related classes of malignant hyperthermia and caffeine, respectively.

For example, the relationship between caffeine and the entry 25435 (mutagen $^{7}$ ) is defined by the entry 0000087 ( has role ${ }^{8}$ ) of the Relations Ontology. This means that the relationship defines that caffeine has role mutagen.

We can also search in the OWL file for the definition of the type of relation has role:

\$ xmllint --xpath "//*[localname ()$=$ 'ObjectProperty'] [@

* [local-name ()$=$ ' about $\left.^{\prime}\right]=$ ' http://purl.obolibrary.org

/obo/RO_0000087']"

chebi_lite.owl

\footnotetext{
${ }^{5}$ http://purl.obolibrary.org/obo/CHEBI_26385

${ }^{6}$ http://purl.obolibrary.org/obo/CHEBI_27134

${ }^{7}$ http://purl.obolibrary.org/obo/CHEBI_25435

${ }^{8}$ http://purl.obolibrary.org/obo/RO_0000087
}

The XPath query starts by finding the elements ObjectProperty and then selects the ones containing the about attribute with the relation URI as value.

We can check that the relation is neither transitive or cyclic:

<owl: ObjectProperty rdf:about=" http: //purl.obolibrary.org/ obo/RO_0000087"> <oboInOwl : hasDbXref rdf : datatype $=$ "http $: / /$ www.w3.org/2001/ XMLSchema\#string" > $\mathrm{RO}: 0000087</$ oboInOwl : hasDbXref > <oboInOwl : hasOBONamespace rdf : datatype $=$ "http $: / /$ www.w3.org/2001/ XMLSchema\#string" > chebi_ontology</ oboInOwl : hasOBONamespace $>$

<oboInOwl: id rdf: datatype= "http://www.w3.org /2001/XMLSchema\#string" $>$ has_role</oboInOwl:id>

<oboInOwl:is_cyclic rdf: datatype $=$ "http $: / /$ www $\cdot$ w3 .org/2001/XMLSchema\# boolean" $>$ false $<$ / oboInOwl:is_cyclic> <oboInOwl: is_transitive rdf : datatype="http $: / /$ www.w3.org/2001/ XMLSchema\#boolean" > false</oboInOwl: is_transitive>

<oboInOwl: shorthand rdf: datatype $=$ "http : / / www . w3 .org/2001/XMLSchema\# string" >has_role $</$ oboInOwl : shorthand> <rdfs:label rdf:datatype=" http://www.w3.org/2001/ XMLSchema\#string" > has role</rdfs: label>

</owl: ObjectProperty> 


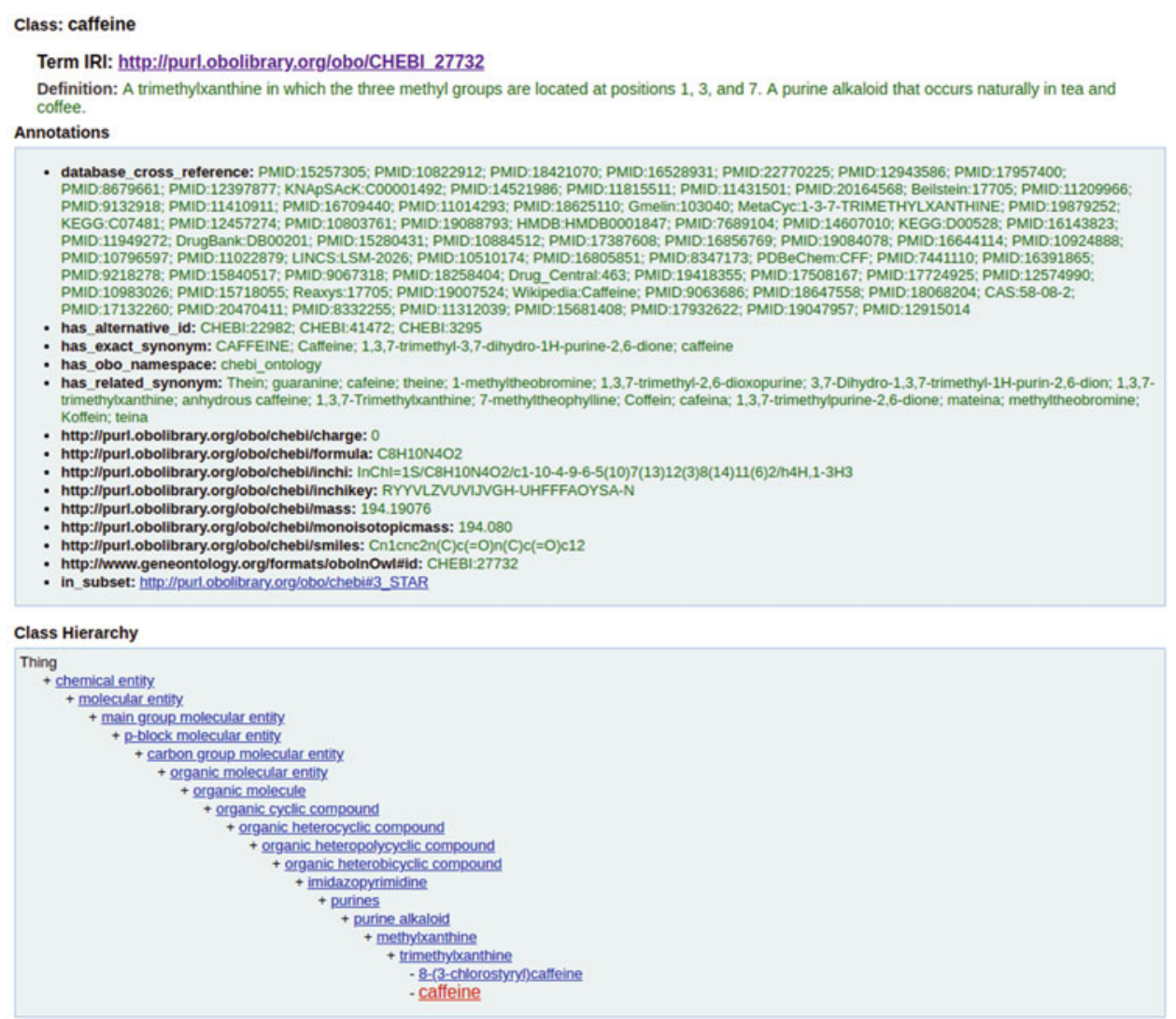

Fig. 5.2 Class description of caffeine in ChEBI (Source: http://www.ontobee.org/)

\section{Superclasses \& Asserted Axioms}

- muscle tissue disease

- autosomal dominant disease

- has material basis in some autosomal dominant inheritance

Fig. 5.3 Related classes of malignant hyperthermia in the Human Disease Ontology (Source: http://www.ontobee. org/)

A graphical visualization of this property is depicted in Fig. 5.5.

\section{URIs and Labels}

In the previous examples, we searched the OWL file using labels and URIs. To standardize the process, we will create two scripts that will convert a label into a URI and vice-versa. The idea is to perform all the internal ontology processing using the URIs and in the end convert them to labels, so we can use them in text processing.

\section{URI of a Label}

To get the URI of malignant hyperthermia, we can use the following query:

$$
\begin{aligned}
& \text { xmllint - -xpath "//*[local- } \\
& \text { name()='label' and text() } \\
& \text { ='malignant hyperthermia } \\
& \text { ']/../@*[local-name }()=\text { ' } \\
& \text { about']" doid.owl }
\end{aligned}
$$

We added the @*[1ocal-name ()$=$ 'about ' ] to extract the URI specified as an attribute of that class.

The output will be the name of the attribute and its value: 


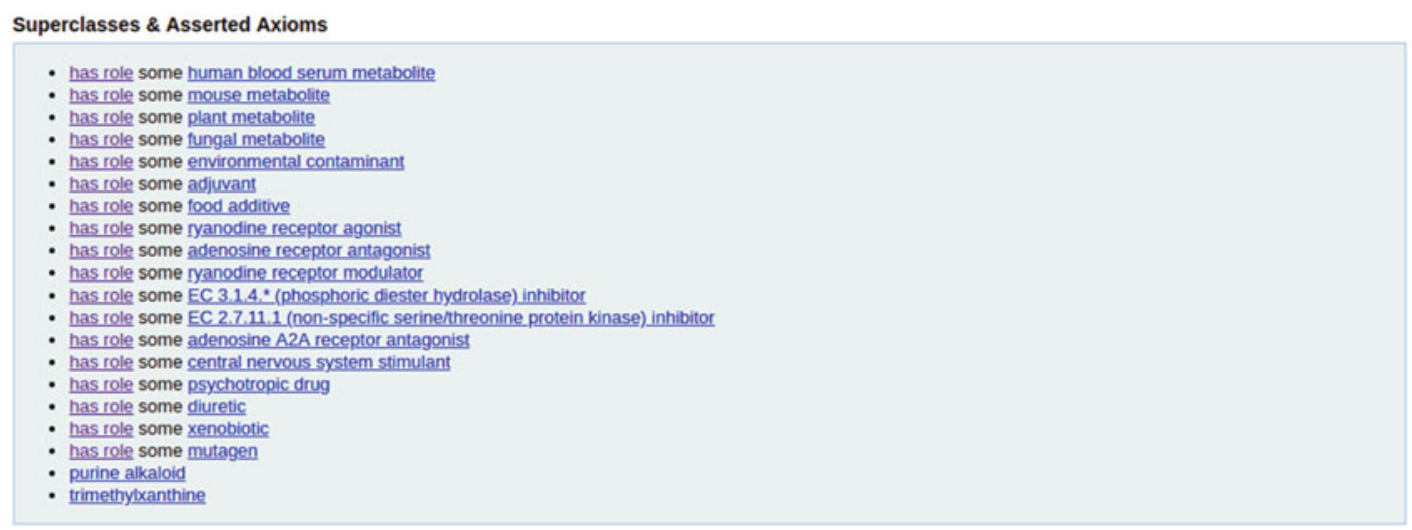

Fig. 5.4 Related classes of caffeine in ChEBI (Source: http://www.ontobee.org/)

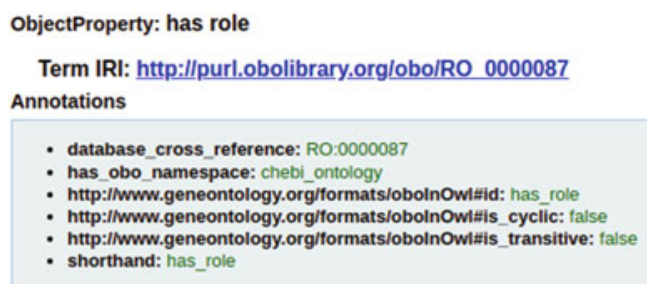

Fig. 5.5 Description of has role property (Source: http://www.ontobee.org/)

rdf : about="http: //purl . obolibrary.org/obo/DOID_8545"

To extract only the value, we can add the string function to the XPath query:

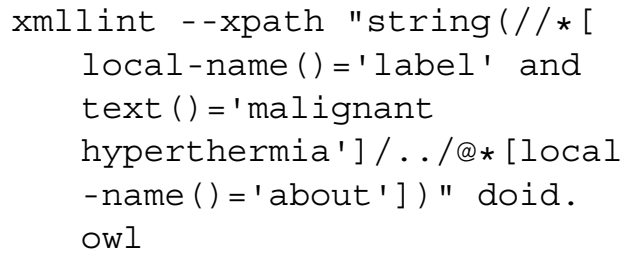

Unfortunately, the string function returns only one attribute value, even if many are matched. Nonetheless, we use the string function because we assume that malignant hyperthermia is an unambiguous label, i.e. only one class will match.

The output will now be only the attribute value:

\section{http://purl.obolibrary.org/obo/ DOID_8545}

To get the URI of caffeine is just about the same command:

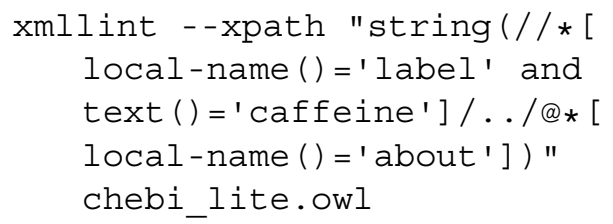

We can now write a script that receives multiple labels given as standard input and the OWL file where to find the URIs as argument. Thus, we can create the script named geturi.sh with the following lines:

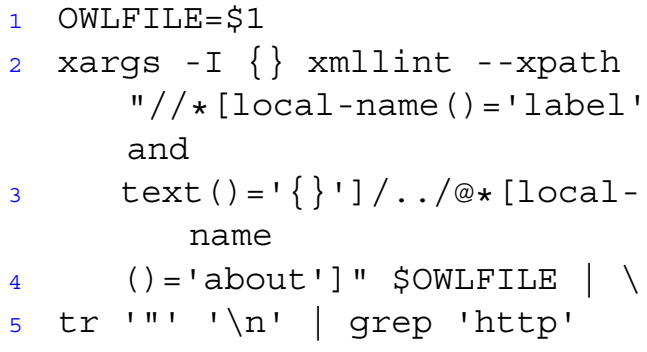

Again we cannot forget to save the file in our working directory, and add the right permissions using chmod as we did with our scripts in the previous chapters. The xargs command is used to process each line of the standard input. The $t r$ 
command was added because xmllint displays all the matches in the same line, so we split the output using the character delimiting the URI, i.e. ". Then we use the grep command to keep only the lines with a URI, i.e. the ones that contain the term http.

Now to execute the script we only need to provide the labels as standard input:

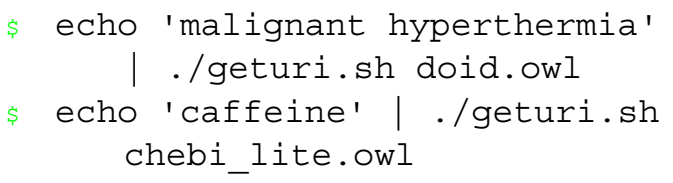

The output should be the URIs of those classes:

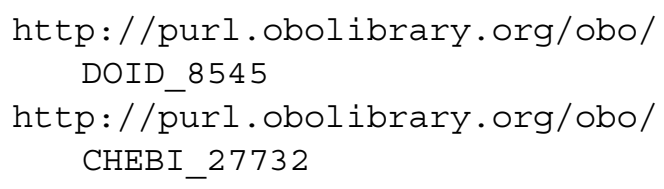

We can also execute the script using multiple labels, one per line:

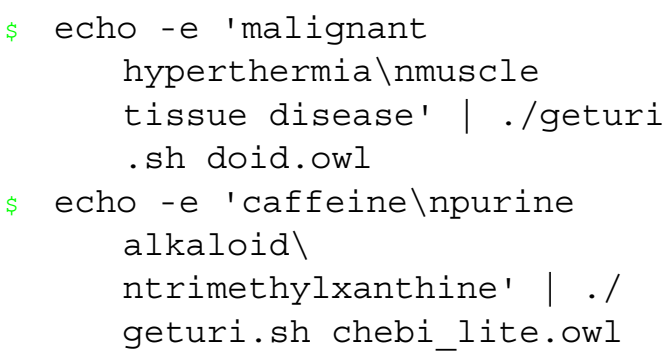

The output will be a URI for each label:

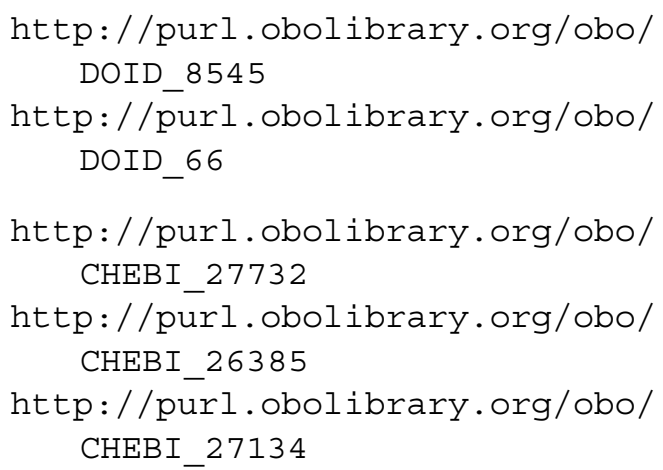

\section{Label of a URI}

To get the label of the disease entry with the identifier 8545, we can also use the xmllint command:

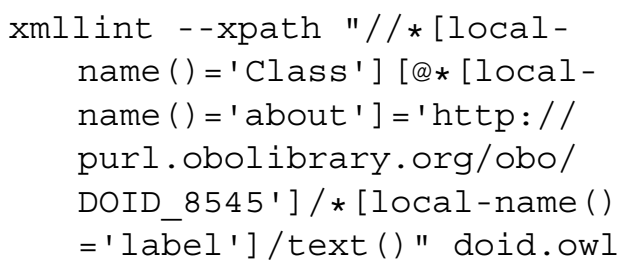

We added the @*[local-name ()$=$ label '] to select the element within the class that describes the label.

The output should be the label we were expecting:

malignant hyperthermia

We can do the same to get the label of the compound entry with the identifier 27732 :

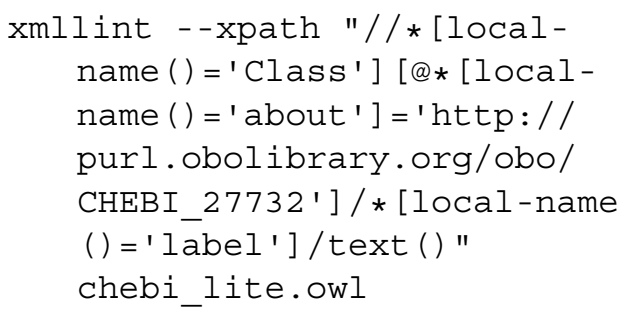

Again, the output should be the label we were expecting:

caffeine

We can now write a script that receives multiple URIs given as standard input and the OWL file where to find the labels. We can create a script named getlabels.sh with the following lines:

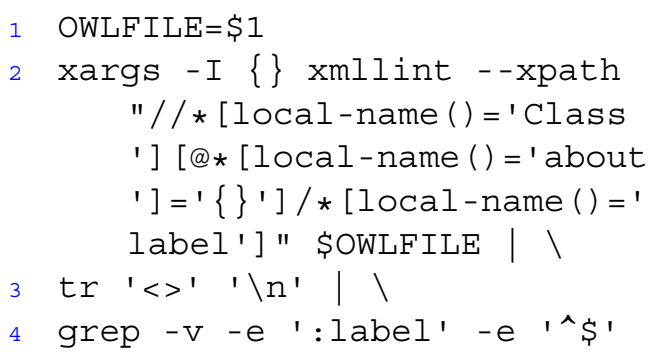

The xargs command is used to process each line of the standard input. The text function does not add a newline character after each match, so if we have multiple matches is almost impossible to separate them. This explains why we removed the text function from the XPath. Then we have to split the result in multiple lines using the $t r$ command and filtering the lines that contain the : label keyword or are empty. 
Now to execute the script we only need to provide the URIs as standard input:

\$ echo 'http://purl.obolibrary. org/obo/DOID_8545' | ./ getlabels.sh doid.owl

echo 'http://purl.obolibrary. org/obo/CHEBI_27732'| . / getlabels.sh chebi_lite. owl

The output should be the labels of those classes:

malignant hyperthermia caffeine

We can also execute the script with multiple URIs:

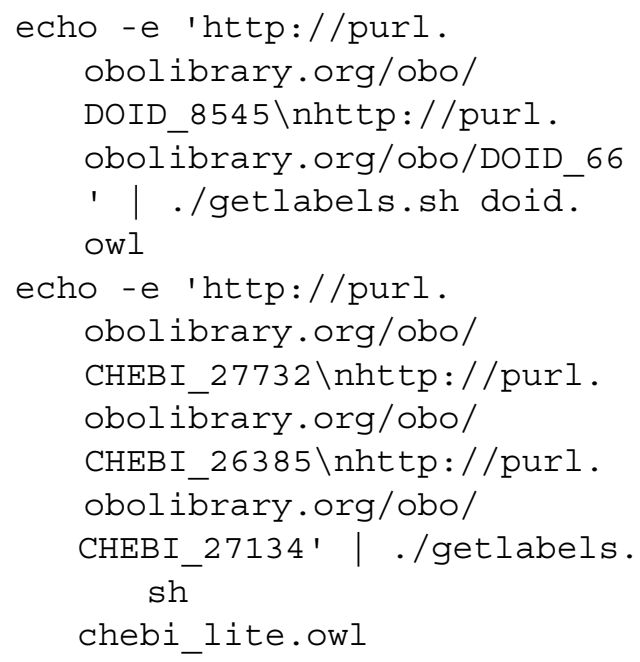

The output will be a label for each URI:

malignant hyperthermia

muscle tissue disease

caffeine

purine alkaloid

trimethylxanthine

To test both scripts, we can feed the output of one as the input of the other, for example:

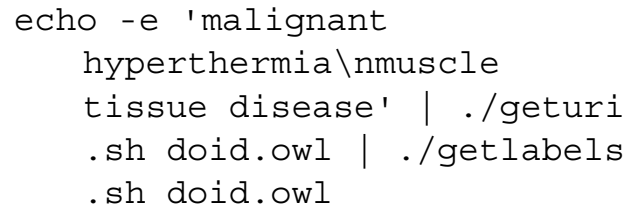

echo -e 'caffeine $\backslash$ npurine alkaloid ntrimethylxanthine' | ./ geturi.sh chebi_lite.owl

| ./getlabels.sh chebi_lite.owl

The output will be the original input, i.e. the labels given as arguments to the echo command:

malignant hyperthermia

muscle tissue disease

\section{caffeine}

purine alkaloid

trimethylxanthine

Now we can use the URIs as input:

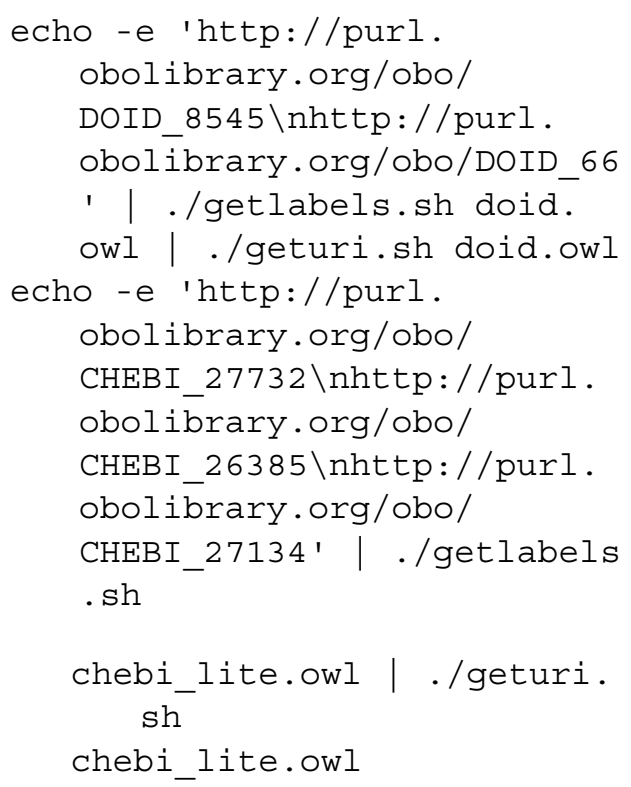

Again the output will be the original input, i.e. the URIs given as arguments to the echo command:

http://purl.obolibrary.org/obo/ DOID 8545

http://purl.obolibrary.org/obo/ DOID_66

http://purl.obolibrary.org/obo/ CHEBI_27732

http://purl.obolibrary.org/obo/ CHEBI_26385

http://purl.obolibrary.org/obo/ CHEBI_27134 


\section{Synonyms}

Concepts are not always mentioned using the same official label. Frequently, we can find in text alternative labels. This is why some of the classes also specify alternative labels, such as the ones represented by the element hasExact Synonym.

For example, to find all the synonyms of a disease, we can use the same XPath as used before but replacing the keyword label by hasExact Synonym:

$$
\begin{aligned}
& \text { xmllint --xpath "//*[local- } \\
& \text { name()='Class'] [@*[local- } \\
& \text { name( ='about'] = 'http:// } \\
& \text { purl.obolibrary.org/obo/ } \\
& \text { DoID_8545']/*[local-name() } \\
& \text { ='hasExactSynonym'] " doid. } \\
& \text { owl }
\end{aligned}
$$

The output will be the two synonyms of $m a$ lignant hyperthermia:

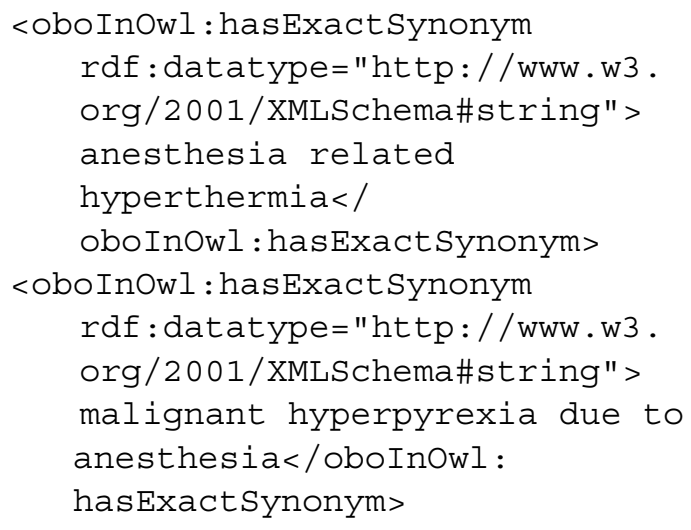

We can also get both the primary label and the synonyms. We only need to add an alternative match to the keyword label:

1 xmllint --xpath "//*[local-

name ()$=$ 'Class' $^{\text {Cl }}$ @* [local-

name ()$=$ ' about' $]=$ ' http: $/ /$

purl.obolibrary.org/obo/

DOID_8545']/*[1ocal-name ()

= 'hasExact Synonym' or

local-name ( ) = 'label'] "

doid.owl

The output will include now the two synonyms plus the official label:
<oboInOwl : has Exact Synonym

rdf : datatype="http $: / /$ www.$w 3$. org/2001/XMLSchema\#string" > anesthesia related

hyperthermia</

oboInOwl : has Exact Synonym>

<oboInOwl : hasexact Synonym rdf : datatype $=$ "http $: / /$ www $\cdot w 3$. org/2001/XMLSchema\#string" > malignant hyperpyrexia due to anesthesia</

oboInOwl : hasExact Synonym> <rdfs:label rdf:datatype="http:

//www.w3 .org/2001/XMLSchema\# string" $>$ malignant

hyperthermia</rdfs: label>

Thus, we can now update the script getlabels.sh to include synonyms:

1 OWLFILE $=\$ 1$

2 xargs -I \{\} xmllint - xpath

$" / / *$ [local-name ()$=$ ' Class

'] [@* [local-name ()$=$ ' about

' $]=$ ' \{\}$\left.^{\prime}\right] /$ * [local -name ()$=$ '

hasExactsynonym' or local-

name $($ ) = ' hasRelatedsynonym '

or local-name ( ) ='label']"

\$OWLFILE | |

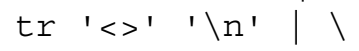

We should note that the XPath query and the grep command were modified by adding the hasExactSynonym keyword. We also added the hasRelatedSynonym which is available for some classes.

We can test the script exactly in the same way as before:

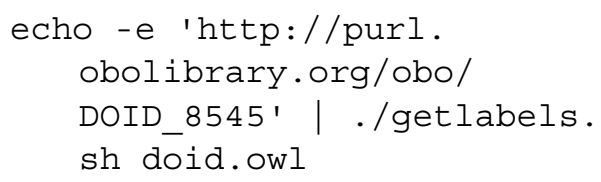

But now the output will display multiple labels for this class:

anesthesia related hyperthermia 
malignant hyperpyrexia due to anesthesia

malignant hyperthermia

\section{URI of Synonyms}

Since the script now returns alternative labels, we may encounter some problems if we send the output to the geturi.sh script:

echo 'http://purl.obolibrary. org/obo/DOID_8545' | ./ getlabels.sh doid.owl | ./ geturi.sh doid.owl

The previous command will display XPath warnings for the two synonyms:

XPath set is empty

XPath set is empty

http: //purl.obolibrary.org/obo/

DOID_8545

If we do not want to know about these mismatches, we can always redirect them to the null device:

echo 'http://purl.obolibrary. org/obo/DOID_8545'| ./ getlabels.sh doid.owl | ./ geturi.sh doid.owl 2>/dev/ null

However, we can update the script geturi.sh to also include synonyms:

1 OWLFILE $=\$ 1$

2 xargs -I \{\} xmllint - xpath

$" / / *[$ (local-name ()$=$ '

hasExactSynonym' or localname ( ) = ' hasRelated.Synonym ' or local-name ()$=$ 'label') and text ()$=$ ' $\left.\}\}^{\prime}\right] / \ldots / @ *[$ local-name ()$=$ ' about' ] " \$OWLFILE | $\mid$

3 tr '"' '\n' | grep 'http'

Now we can execute the same command:

echo 'http://purl.obolibrary. org/obo/DOID_8545'| ./ getlabels.sh doid.owl | ./ geturi.sh doid.owl
Every label should now be matched exactly with the same class:

http: //purl.obolibrary.org/obo/ DOID_8545

http://purl.obolibrary.org/obo/ DOID_8545

http://purl.obolibrary.org/obo/ DOID_8545

If we want to avoid repetitions, we can add the sort command with the $-u$ option to the end of each command, as we did in previous chapters:

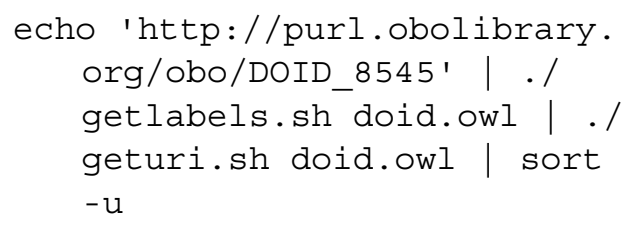

The output should now be only one URI:

http://purl.obolibrary.org/obo/ DOID_8545

\section{Parent Classes}

Parent classes represent generalizations that may also be relevant to recognize in text. To extract all the parent classes of malignant hyperthermia, we can use the following XPath query:

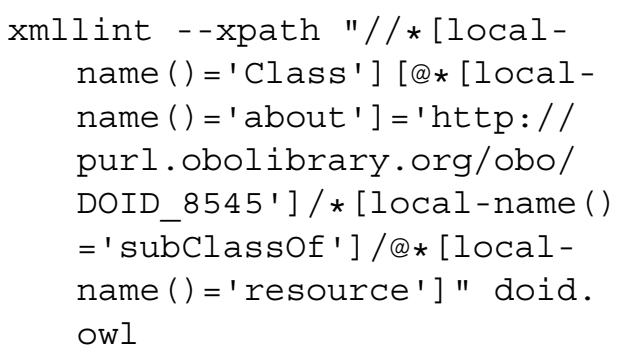

The first part of the XPath is the same as the above to get the class element, then [localname ( ) = 'subClassOf'] is used to get the subclass element, and finally @* [1ocal-name ()$=$ 'resource' ] is used to get the attribute containing its URI.

The output should be the URIs representing the parents of class 8545 :

rdf : resource="http $: / /$ purl .

obolibrary.org/obo/

DOID_0050736" 
rdf : resource="http: / / purl . obolibrary.org/obo/DOID_66"

We can also execute the same command for caffeine:

$$
\begin{aligned}
& \text { xmllint - -xpath "//*[local- } \\
& \text { name()='Class'] [@*[local- } \\
& \text { name()='about'] = 'http:// } \\
& \text { purl.obolibrary.org/obo/ } \\
& \text { CHEBI_27732']/*[local-name } \\
& \text { ()='subclassof']/@*[local- } \\
& \text { name()='resource'] " } \\
& \text { chebi_lite.owl }
\end{aligned}
$$

The output will now include two parents:

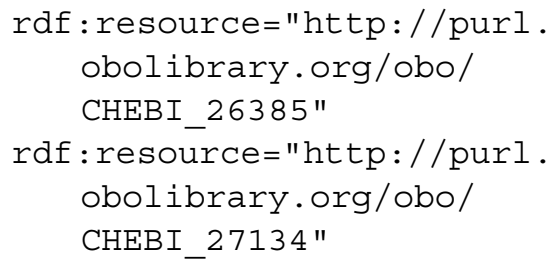

We should note that we no longer can use the string function, because ontologies are organized as DAGs using multiple inheritance, i.e. each class can have multiple parents, and the string function only returns the first match. To get only the URIs, we can apply the previous technique of using the $t r$ and grep commands:

$$
\begin{aligned}
& \text { xmllint --xpath "//*[local- } \\
& \text { name ()='Class'] [@*[local- } \\
& \text { name ()='about'] ='http:// } \\
& \text { purl.obolibrary.org/obo/ } \\
& \text { CHEBI_27732']/*[local-name } \\
& \text { ()='subClassof']/@*[local- } \\
& \text { name()='resource']" } \\
& \text { chebi_lite.owl | tr '"' ' } \\
& \text { n' | grep 'http' }
\end{aligned}
$$

Now the output only contains the URIs:

http: //purl.obolibrary.org/obo/

CHEBI_26385

http://purl.obolibrary.org/obo/

CHEBI_27134

We can now create a script that receives multiple URIs given as standard input and the OWL file where to find all the parents as argument.
The script named getparents.sh should contain the following lines:

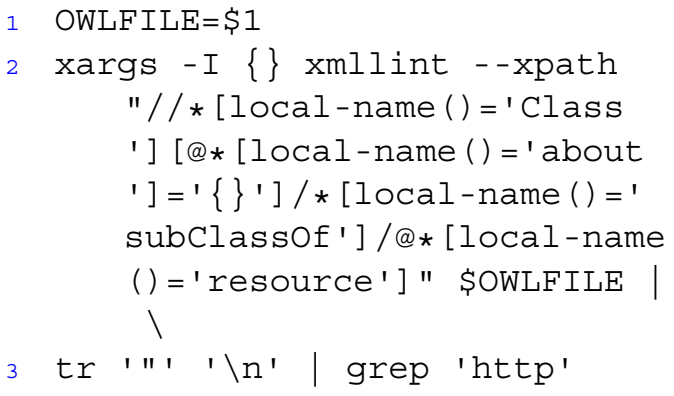

To get the parents of malignant hyperthermia, we will only need to give the URI as input and the OWL file as argument:

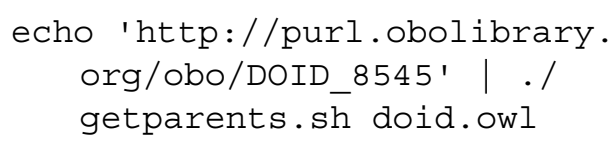

The output will include the URIs of the two parents:

http: //purl.obolibrary.org/obo/ DOID_0050736

http://purl.obolibrary.org/obo/ DOID_66

\section{Labels of Parents}

But if we need the labels we can redirect the output to the getlabels.sh script:

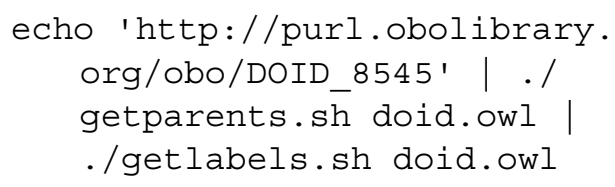

The output will now be the label of the parents of malignant hyperthermia:

autosomal dominant disease muscle tissue disease

Again, the same can be done with caffeine:

echo 'http://purl.obolibrary. org/obo/CHEBI_27732'| ./ getparents.sh chebi_lite. owl . /getlabels.sh chebi_lite.owl 
And now the output contains the labels of the parents of caffeine:

purine alkaloid

trimethylxanthine

\section{Related Classes}

If we are interested in using all the related classes besides the ones that represent a generalization (subClassOf), we have to change our XPath to:

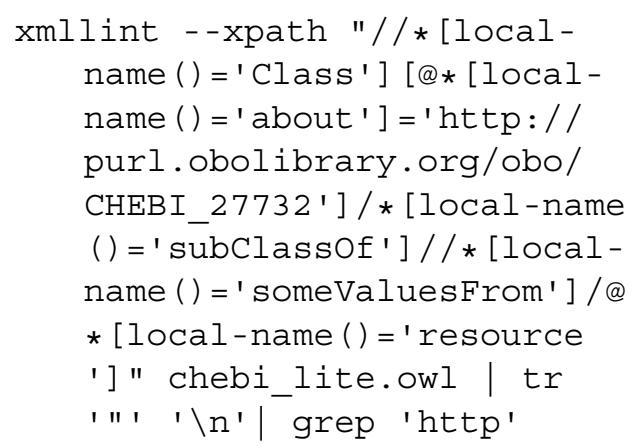

We should note that these related classes are in the attribute resource of someValuesFrom element inside a subClass Of element.

The URIs of the 18 related classes of caffeine are now displayed:

http: //purl.obolibrary.org/obo/ CHEBI_25435

http://purl.obolibrary.org/obo/ CHEBI_35337

http://purl.obolibrary.org/obo/ CHEBI_35471

http://purl.obolibrary.org/obo/ CHEBI 35498

http://purl.obolibrary.org/obo/

CHEBI_35703

http://purl.obolibrary.org/obo/

CHEBI_38809

http://purl.obolibrary.org/obo/

CHEBI_50218

http://purl.obolibrary.org/obo/

CHEBI_50925

http://purl.obolibrary.org/obo/

CHEBI_53121

http: //purl.obolibrary.org/obo/

CHEBI_60809 http: //purl.obolibrary.org/obo/ CHEBI_64047

http://purl.obolibrary.org/obo/ CHEBI_67114

http://purl.obolibrary.org/obo/ CHEBI_71232

http://purl.obolibrary.org/obo/ CHEBI_75771

http://purl.obolibrary.org/obo/ CHEBI 76924

http://purl.obolibrary.org/obo/ CHEBI_76946

http://purl.obolibrary.org/obo/ CHEBI_78298

http://purl.obolibrary.org/obo/ CHEBI_85234

\section{Labels of Related Classes}

To get the labels of these related classes, we only need to add the getlabels.sh script:

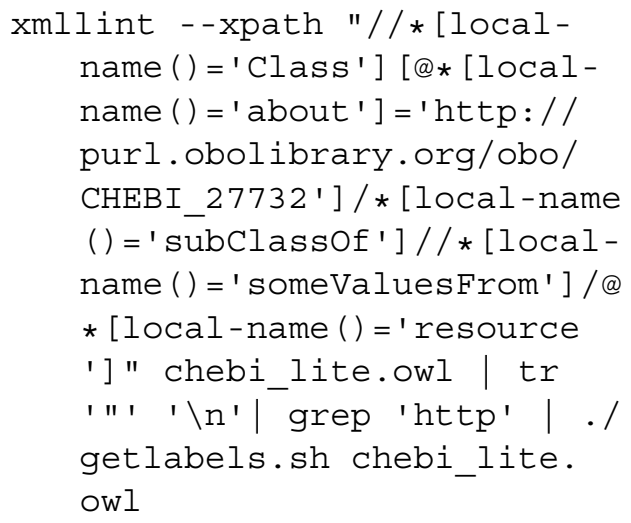

The output is now 18 terms that we could use to expand our text processing:

mutagen

central nervous system stimulant psychotropic drug

diuretic

xenobiotic

ryanodine receptor modulator

EC 3.1.4.* (phosphoric diester hydrolase) inhibitor

EC 2.7.11.1 (non-specific serine /threonine protein kinase) inhibitor 


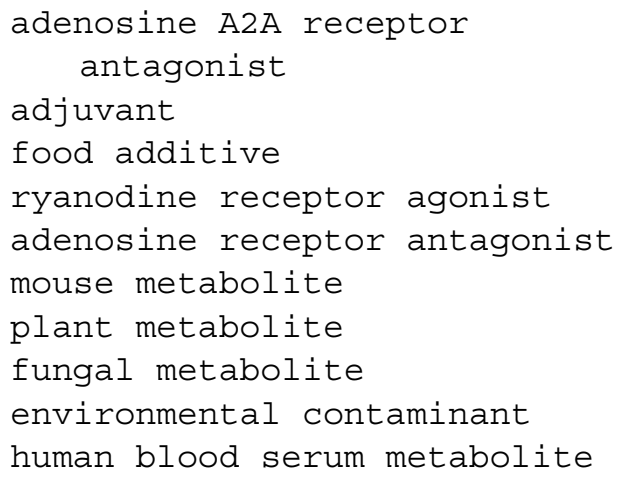

\section{Ancestors}

Finding all the ancestors of a class includes many chain invocations of the getparents.sh until we get no matches. We also should avoid relations that are cyclic, otherwise we will enter in a infinite loop. Thus, for identifying the ancestors of a class, we will only consider parent relations, i.e. subsumption relations.

\section{Grandparents}

In the previous section we were able to extract the direct parents of a class, but the parents of these parents also represent generalizations of the original class. For example, to get the parents of the parents (grandparents) of malignant hyperthermia we need to invoke getparents.sh twice:

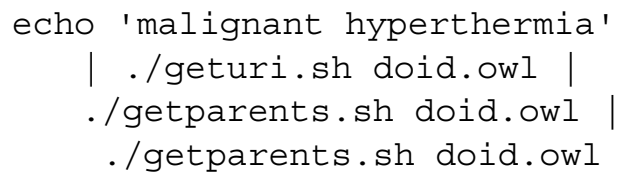

And we will find the URIs of the grandparents of malignant hyperthermia:

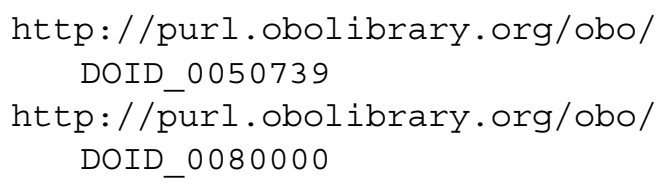

Or to get their labels we can add the getlabels.sh script:

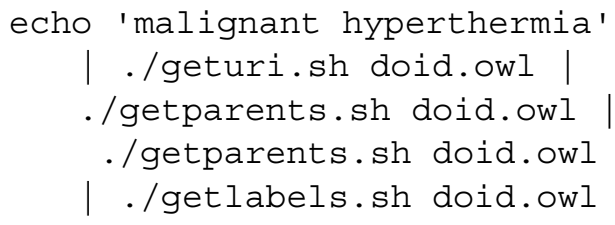

And we find the labels of the grandparents of malignant hyperthermia:

autosomal genetic disease

muscular disease

\section{Root Class}

However, there are classes that do not have any parent, which are called root classes. In Figs. 5.1 and 5.2, we can see that disease and chemical entity are root classes of DO and ChEBI ontologies, respectively. As we can see these are highly generic terms.

To check if it is the root class, we can ask for their parents:

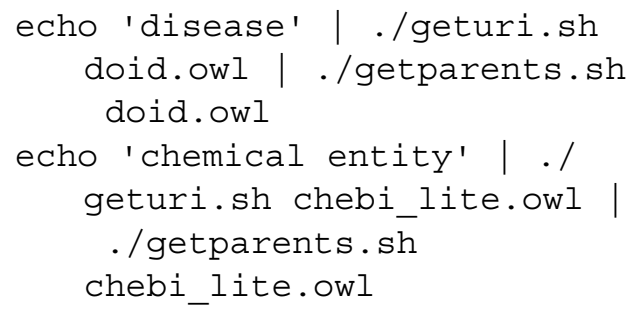

In both cases, we will get the warning that no matches were found, confirming that they are the root class.

XPath set is empty

\section{Recursion}

We can now build a script that receives a list of URIs as standard input, and invokes getparents.sh recursively until it reaches the root class.

The script named getancestors.sh should contain the following lines:

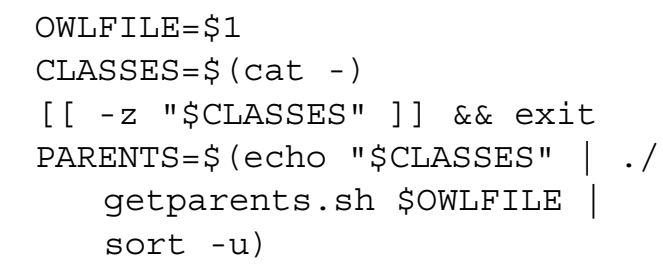


5 echo "\$PARENTS"

6 echo "\$PARENTS" | . /

getancestors.sh \$OWLFILE

The second line of the script saves the standard input in a variable named CLASSES, because we need to use it twice: (i) to check if the input as any classes or is empty (third line) and (ii) to get the parents of the classes given as input (fourth line). If the input is empty then the script ends, this is the base case of the recursion ${ }^{9}$. This is required so the recursion stops at a given point. Otherwise, the script would run indefinitely until the user stops it manually.

The fourth line of the script stores the output in a variable named PARENTS, because we need also to use it twice: (i) to output these direct parents (fifth line), and (ii) to get the ancestors of this parents (sixth line). We should note that we are invoking the getancestors.sh script inside the getancestors.sh, which defines the recursion step. Since the subsumption relation is acyclic, we expect that at some time we will reach classes without parents (root classes) and then the script will end.

We should note that the echo of the variables CLASSES and PARENTS need to be inside commas, so the newline characters are preserved.

\section{Iteration}

Recursion is most of the times computational expensive, but usually it is possible to replace recursion with iteration to develop a more efficient algorithm. Explaining iteration and how to refactor a recursive script is out of scope of this book, nevertheless the following script represents an equivalent way to get all the ancestors without using recursion:

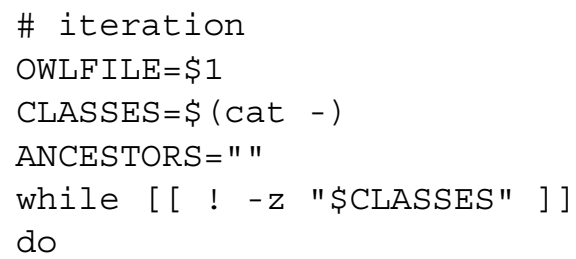

${ }^{9}$ https://en.wikipedia.org/wiki/Recursion
7

8 10 11

The script uses the while command that basically implements iteration by repeating a set of commands (lines 6-8) while a given condition is satisfied (line 4).

To test the recursive script, we can provide as standard input the label malignant hyperthermia:

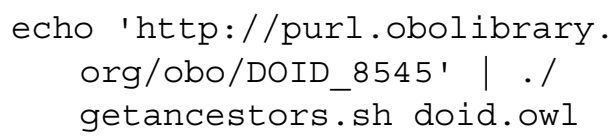

The output will be the URIs of all its ancestors:

http://purl.obolibrary.org/obo/ DOID_0050736

http://purl.obolibrary.org/obo/ DOID_66

http://purl.obolibrary.org/obo/ DOID_0050739

http://purl.obolibrary.org/obo/ DOID_0080000

http://purl.obolibrary.org/obo/ DOID_0050177

http://purl.obolibrary.org/obo/ DOID 17

http://purl.obolibrary.org/obo/ DOID_630

http://purl.obolibrary.org/obo/ DOID 7

http://purl.obolibrary.org/obo/ DOID_4

We should note that we will still receive the XPath warning when the script reaches the root class and no parents are found:

XPath set is empty

To remove this warning and just get the labels of the ancestors of malignant hyperthermia, we can redirect the warnings to the null device:

echo 'malignant hyperthermia' ./geturi.sh doid.owl 


$$
\begin{aligned}
& \text {./getancestors.sh doid.owl } \\
& 2>/ \text { dev/null | ./getlabels } \\
& \text {.sh doid.owl }
\end{aligned}
$$

The output will now include the name of all ancestors of malignant hyperthermia:

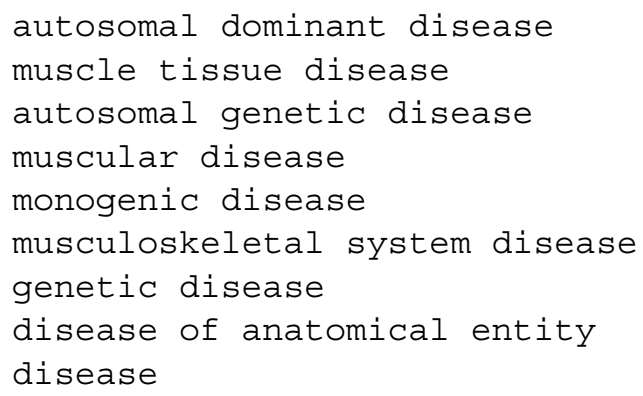

We should note that the first two ancestors are the direct parents of malignant hyperthermia, and the last one is the root class. This happens because the recursive script print the parents before invoking itself to find the ancestors of the direct parents.

We can do the same with caffeine, but be advised that given the higher number of ancestors in ChEBI we may now have to wait a little longer for the script to end.

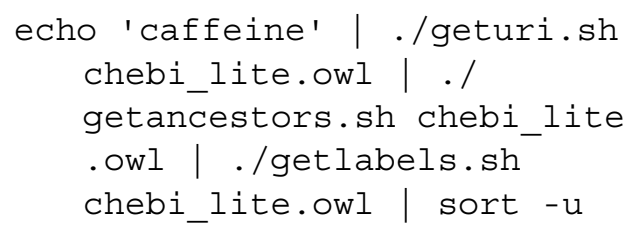

The results include repeated classes that were found by using different branches, so that is why we need to add the sort command with the $-u$ option to eliminate the duplicates.

The script will print the ancestors being found by the script:

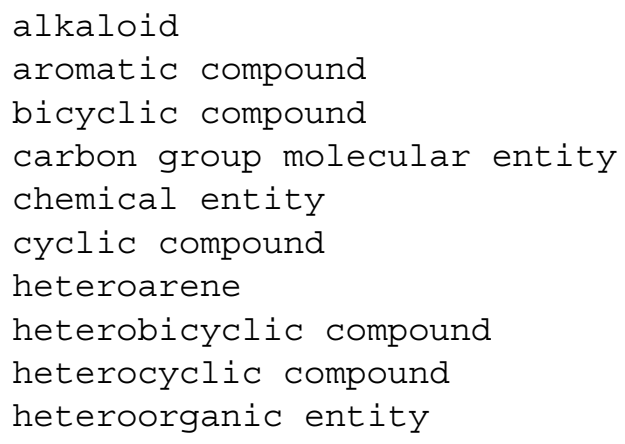

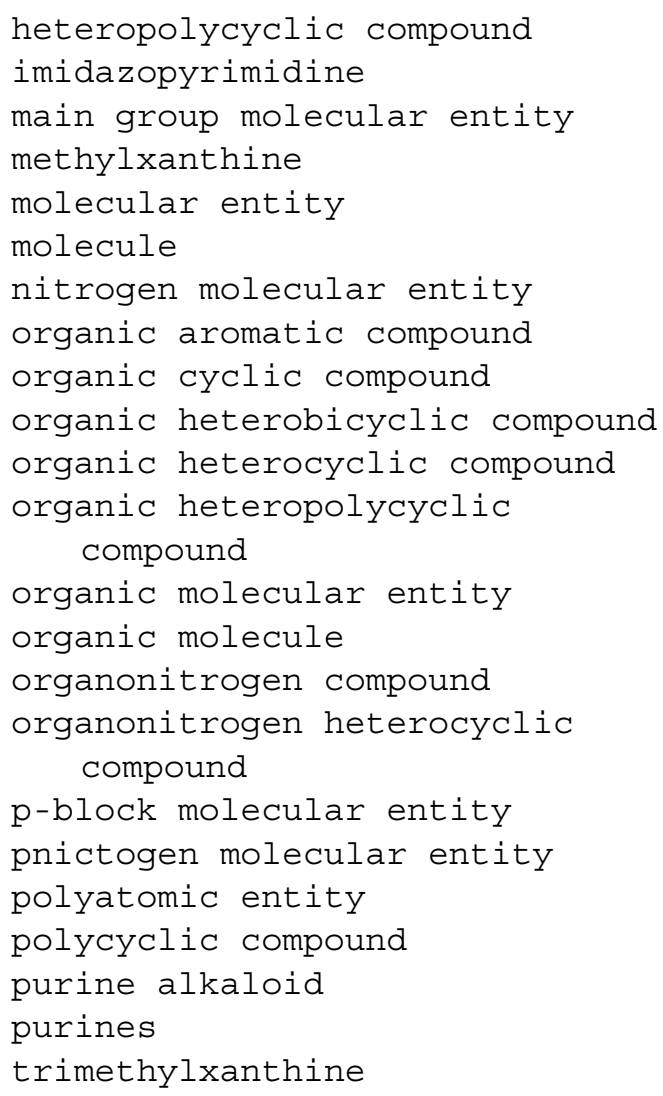

\section{My Lexicon}

Now that we know how to extract all the labels and related classes from an ontology, we can construct our own lexicon with the list of terms that we want to recognize in text.

Let us start by creating the file do_8545_ lexicon.txt representing our lexicon for malignant hyperthermia with all its labels:

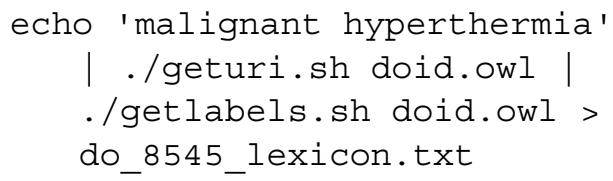

\section{Ancestors Labels}

Now we can add to the lexicon all the labels of the ancestors of malignant hyperthermia by adding the redirection operator: 


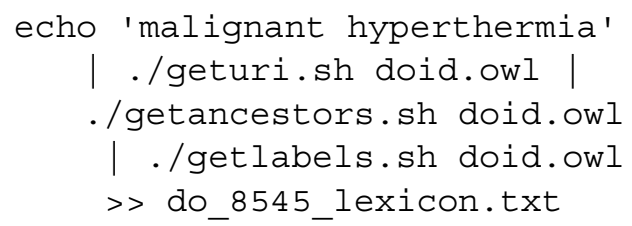

We should note that now we use $>>$ and not $>$, this will append more lines to the file instead of creating a new file from scratch.

Now we can check the contents of the file do_8545_lexicon.txt to see the terms we got:

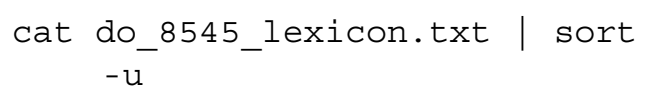

We should note that we use the sort command with the $-u$ option to eliminate any duplicates that may exist.

We should be able to see the following labels: anesthesia related hyperthermia autosomal dominant disease autosomal genetic disease disease

disease of anatomical entity genetic disease

malignant hyperpyrexia due to anesthesia

malignant hyperthermia

monogenic disease

muscle tissue disease

muscular disease

musculoskeletal system disease

We can also apply the same commands for caffeine to produce its lexicon in the file chebi_27732_lexicon.txt by adding the redirection operator:

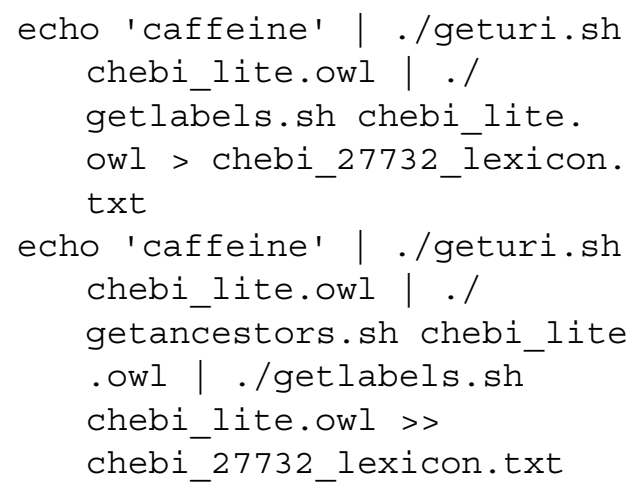

We should note that it may take a while until it gets all labels.
Now let us check the contents of this new lexicon:

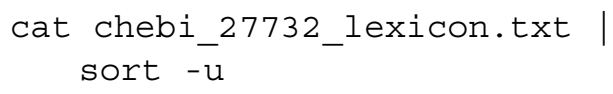

Now we should be able to see that this lexicon is much larger:

alkaloid

aromatic compound

bicyclic compound

caffeine

...

\section{Merging Labels}

If we are interested in finding everything related to caffeine or malignant hyperthermia, we may be interested in merging the two lexicons in a file named lexicon.txt:

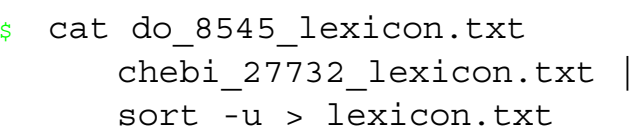

Using this new lexicon, we can recognize any mention in our previous file named chebi_27732_sentences.txt:

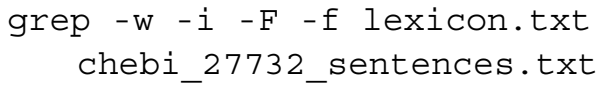

We added the $-F$ option because our lexicon is a list of fixed strings, i.e. does not include regular expressions. The equivalent long form to the $-F$ option is - -fixed-strings.

We now get more sentences, including some that do not include a direct mention to caffeine or malignant hyperthermia. For example, the following sentence was selected because it mentions molecule, which is an ancestor of caffeine:

The remainder of the molecule is hydrophilic and presumably constitutes the cytoplasmic domain of the protein.

Another example is the following sentence, which was selected because it mentions disease, which is an ancestor of malignant hyperthermia: 


\section{Our data suggest that divergent activity profiles may cause varied disease phenotypes by specific mutations.}

We can also use our script getentities.sh giving this lexicon as argument. However, since we are not using any regular expressions it would be better to add the $-\mathrm{F}$ option to the grep command in the script, so the lexicon is interpreted as list of fixed strings to be matched. Only then we can execute the script safely:

./getentities.sh lexicon.txt < chebi_27732_sentences.txt

\section{Ancestors Matched}

Besides these two previous examples, we can check if there other ancestors being matched by using the grep command with the - o option:

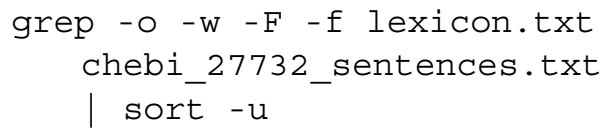

We can see that besides the terms caffeine and malignant hyperthermia, only one ancestor of each one of them was matched, molecule and disease, respectively:

caffeine

disease

malignant hyperthermia

molecule

This can be explained because our text is somehow limited and because we are using the official labels and we may be missing acronyms, and simple variations such as the plural of a term. To cope with this issue, we may use a stemmer ${ }^{10}$, or use all the ancestors besides subsumption. However, if our lexicon is small is better to do it manually and maybe add some regular expressions to deal with some of the variations.

\footnotetext{
${ }^{10}$ https://en.wikipedia.org/wiki/Stemming
}

\section{Generic Lexicon}

Instead of using a customized and limited lexicon, we may be interested in recognizing any of the diseases represented in the ontology. By recognizing all the diseases in our caffeine related text, we will be able to find all the diseases that may be related to caffeine

\section{All Labels}

To extract all the labels from the disease ontology we can use the same XPath query used before, but now without restricting it to any URI:

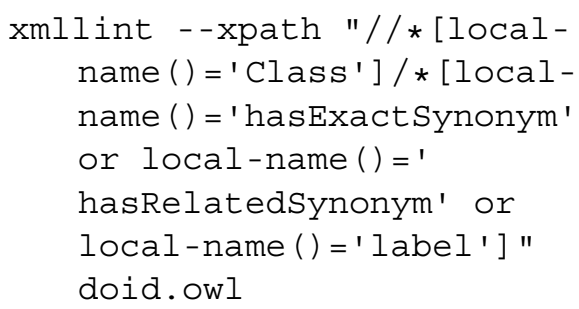

We can create a script named getalllabels.sh, that receives as argument the OWL file where to find all labels containing the following lines:

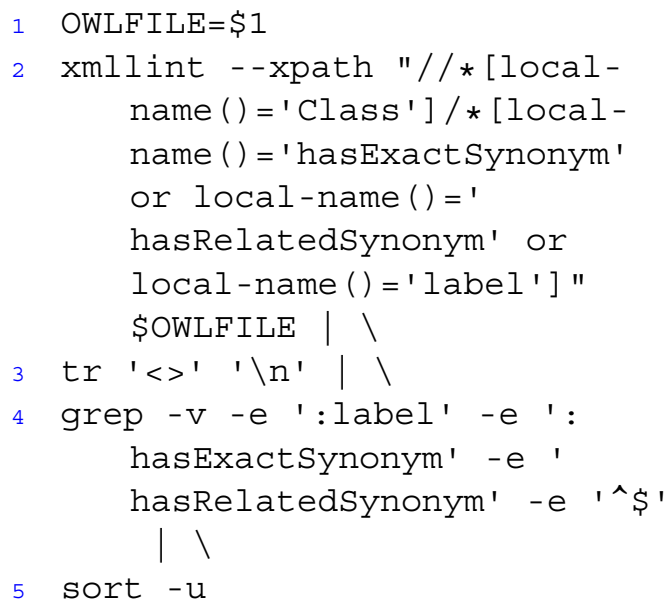

We should note that this script is similar to the getlabels.sh script without the xargs, since it does not receive a list of URIs as standard input.

Now we can execute the script to extract all labels from the OWL file:

./getalllabels.sh doid.owl

The output will contain the full list of diseases: 


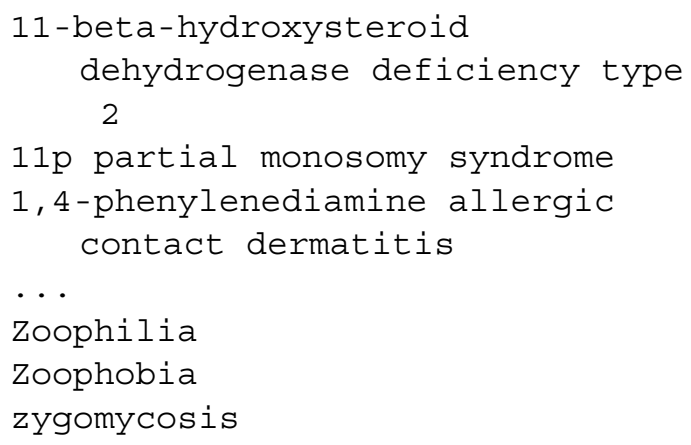

To create the generic lexicon, we can redirect the output to the file diseases.txt:

\section{-/getalllabels.sh doid.owl > diseases.txt}

We can check how many labels we got by using the wc command:

\section{wc - 1 diseases.txt}

The lexicon contains more than 29 thousand labels.

We can now recognize the lexicon entries in the sentences of the file chebi_27732_sentences.txt by using the grep command:

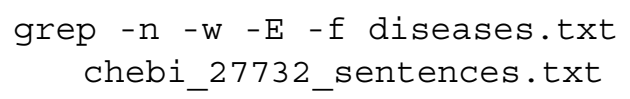

However, we will get the following error:

grep: Unmatched ) or \)

This error happens because our lexicon contains some special characters also used by regular expressions, such as the parentheses.

One way to address this issue is to replace the $-E$ option by the $-F$ option, that treats each lexicon entry as a fixed string to be recognized:

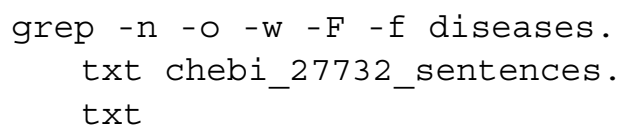

The output will show the large list of sentences mentioning diseases:

1:malignant hyperthermia

2:malignant hyperthermia

9: central core disease

10 : disease

10 : myopathy 1092:malignant hyperthermia

1092: central core disease

1103 :malignant hyperthermia

1104 :malignant hyperthermia

1106: central core disease

1106 :myopathy

\section{Problematic Entries}

Despite using the $-F$ option, the lexicon contains some problematic entries. Some entries have expressions enclosed by parentheses or brackets, that represent alternatives or a category:

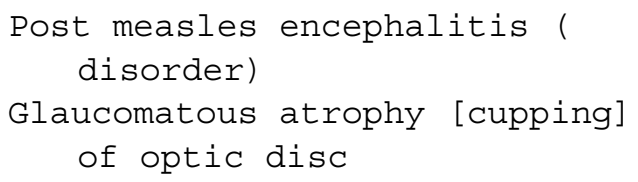

Other entries have separation characters, such as commas or colons, to represent a specialization. For example:

Tapeworm infection: intestinal

taenia solum

Tapeworm infection: pork

Pemphigus, Benign Familial

ATR, nondeletion type

A problem is that not all have the same meaning. A comma may also be part of the term. For example:

46, XY DSD due to LHB deficiency

Other case includes using \&amp; to represent an ampersand. For example:

Gonococcal synovitis \&amp;/or tenosynovitis

However, most of the times the alternatives are already included in the lexicon in different lines. For example:

Gonococcal synovitis and tenosynovitis

Gonococcal synovitis or tenosynovitis

As we can see by these examples, it is not trivial to devise rules that fully solve these issues. Very likely there will be exceptions to any rule we devise and that we are not aware of. 


\section{Special Characters Frequency}

To check the impact of each of these issues, we can count the number of times they appear in the lexicon:

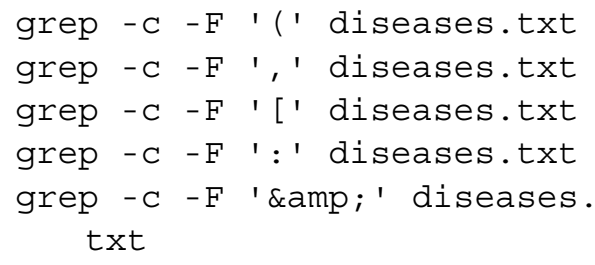

We will be able to see that parentheses and commas are the most frequent, with more than one thousand entries.

\section{Completeness}

Now let us check if the ATR acronym representing the alpha thalassemia-X-linked intellectual disability syndrome is in the lexicon:

\section{grep -E '^ATR' diseases.txt}

All the entries include more terms than only the acronym:

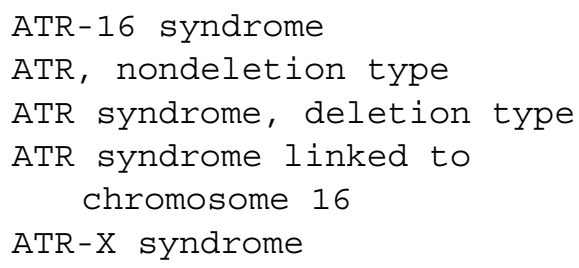

Thus, a single ATR mention will not be recognized.

This is problematic if we need to match sentences mentioning that acronym, such as:

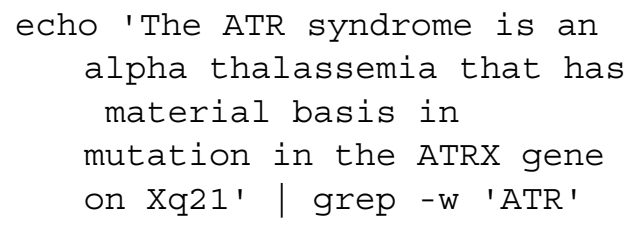

We will now try to mitigate these issues as simply as we can. We will not try to solve them completely, but at least address the most obvious cases.

\section{Removing Special Characters}

The first fix we will do, is to remove all the parentheses and brackets by using the tr command, since they will not be found in the text:

\$ tr - d ' [ ] () \{\}$^{\prime}<$ diseases.txt

Of course, we may lose the shorter labels, such as Post measles encephalitis, but at least now, the disease Post measles encephalitis disorder will be recognized:

$$
\begin{gathered}
\text { tr - d ' [] ()\{\}'< diseases.txt } \\
\text { | grep 'Post measles } \\
\text { encephalitis disorder' }
\end{gathered}
$$

If we really need these alternatives, we would have to create multiple entries in the lexicon or transform the labels in regular expressions.

\section{Removing Extra Terms}

The second fix is to remove all the text after a separation character, by using the sed command:

$$
\begin{gathered}
\text { tr }-d{ }^{\prime}[]()\{\} '<\text { diseases.txt } \\
\mid \text { sed }-E \text { 's/ }[,: ;] . * \$ / / 1
\end{gathered}
$$

We should note that the regular expression enforces a space after the separation character to avoid separation characters that are not really separating two expressions, such as: $46, X Y D S D$ due to LHB deficiency

We can see that now we are able to recognize both ATR and ATR syndrome:

$$
\begin{gathered}
\text { tr }-d{ }^{\prime}[]()\{\} '<\text { diseases.txt } \\
\text { | sed }-E \text { 's } /[,: ;] . * \$ / / \text { | } \\
\text { grep -E 'AATR' }
\end{gathered}
$$

\section{Removing Extra Spaces}

The third fix is to remove any leading or trailing spaces of a label:

$$
\begin{gathered}
\text { tr }-d{ }^{\prime}[]()\{\} '<\text { diseases.txt } \\
\mid \text { sed }-\mathrm{E} ' \mathrm{~s} /[,: ;] . * \$ / / ; \mathrm{s} \\
/ \wedge * / / ; \mathrm{s} / * \$ / / 1
\end{gathered}
$$

We should note that we added two more replacement expressions to the sed command by separating them with a semicolon. 
We can now update the script getallabels.sh to include the previous tr and sed commands:

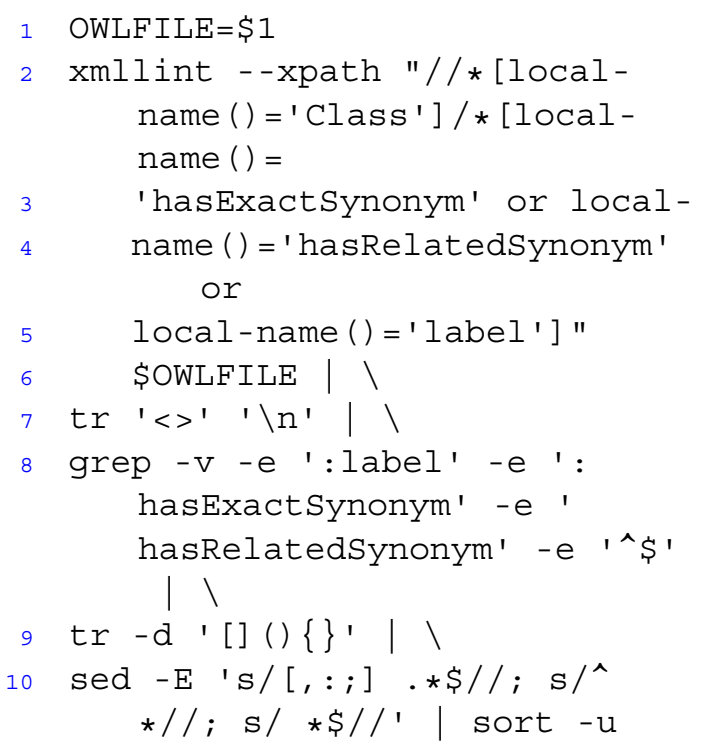

And we can now generate a fixed lexicon:

\section{./getalllabels.sh doid.owl > diseases.txt}

We can check again the number of entries:

wc - 1 diseases.txt

We now have a lexicon with about 28 thousand labels. We have less entries because our fixes made some entries equal to others already in the lexicon, and thus the - $u$ option filtered them.

\section{Disease Recognition}

We can now try to recognize lexicon entries in the sentences of file chebi_27732_ sentences.txt:

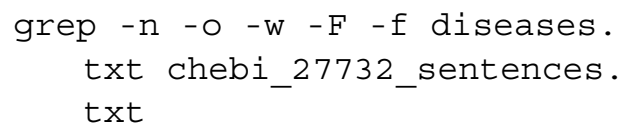

To obtain the list of labels that were recognized, we can use the grep command:

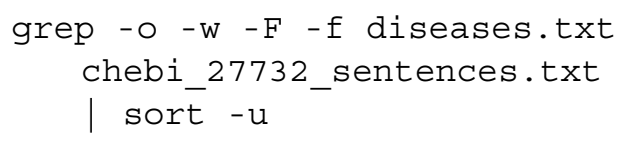

We will get a list of 43 unique labels representing diseases that may be related to caffein:

Andersen-Tawil syndrome

arrhythmogenic right ventricular cardiomyopathy

ARVD2

ataxia telangiectasia

ATR

atrial fibrillation

benign congenital myopathy

cancer

cardiac arrest

cardiomyopathy

catecholaminergic polymorphic

ventricular tachycardia

central core disease

chorea

congenital hip dislocation

congenital myopathy

deficiency

disease

dystonia

epilepsy

FHL1

hand

hepatitis C

HL

hypercholesterolaemia

hypokalemic periodic paralysis

Hypokalemic periodic paralysis

intellectual disability

long QT syndrome

LQT1

LQT2

LQT3

LQT5

LQT6

malignant hyperthermia

migraine

myopathy

myotonic dystrophy type 1

nemaline myopathy

nemaline rod myopathy

ophthalmoplegia

rod myopathy

scoliosis

syndrome 


\section{Performance}

The grep is quite efficient but of course when using large lexicons and texts we may start to feel some performing issues. Its execution time is proportional to the size of the lexicon, since each term of the lexicon will correspond to an independent pattern to match. This means that for large lexicons we may face serious performance issues.

\section{Inverted Recognition}

A solution for dealing with large lexicons is to use the inverted recognition technique (Couto et al. 2017; Couto and Lamurias 2018). The inverted recognition uses the words of the input text as patterns to be matched against the lexicon file. When the number of words in the input text is much smaller than the number of terms in the lexicon, grep has much fewer patterns to match. For example, the inverted recognition technique applied to ChEBI has shown to be more than 100 times faster than using the standard technique.

\section{Case Insensitive}

Another performance issue arises when we use the $-i$ option to perform a case insensitive matching. For instance, in most computers if we execute the following command, we will have to wait much longer than not using the - $i$ option:

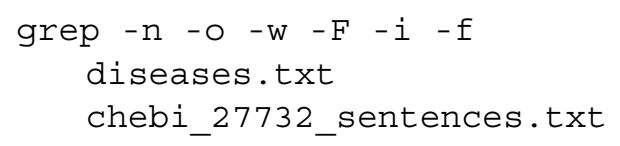

One solution is to convert both the lexicon and text to lowercase (or uppercase), but this may result in more incorrect matches, such as incorrectly matching acronyms in lowercase.

\section{ASCII Encoding}

The low performance issue of case insensitive matching is normally due to the usage of UTF-8 character encoding ${ }^{11}$, instead of ASCII character

\footnotetext{
${ }^{11}$ https://en.wikipedia.org/wiki/UTF-8
}

encoding ${ }^{12}$. UTF-8 allow us to use special characters, such as the euro symbol, in a standard way so it is interpreted by every computer around the world in the same way. However, for normal text without special characters ASCII works fine and more efficiently. In Unix shells we can normally specify the usage of ASCII encoding by adding the expression LC_ALL=C before the command (man locale for more information).

So, another solution is to execute the following command:

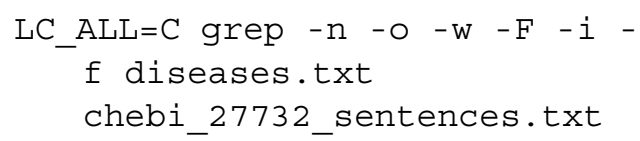

We will be able to watch the significant increase in performance.

To check how many labels are now being recognized we can execute:

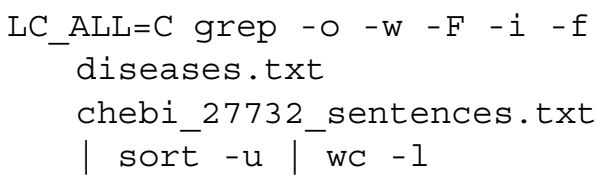

We have now 60 labels being recognized.

To check which new labels were recognized, we can compare the results with and without the - i option:

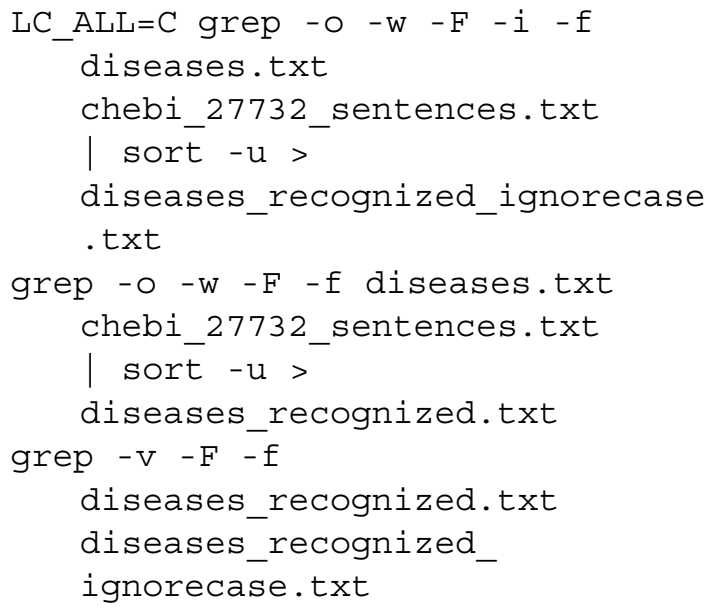

We are now able to see that the new labels are:

${ }^{12} \mathrm{https}: / /$ en.wikipedia.org/wiki/ASCII 


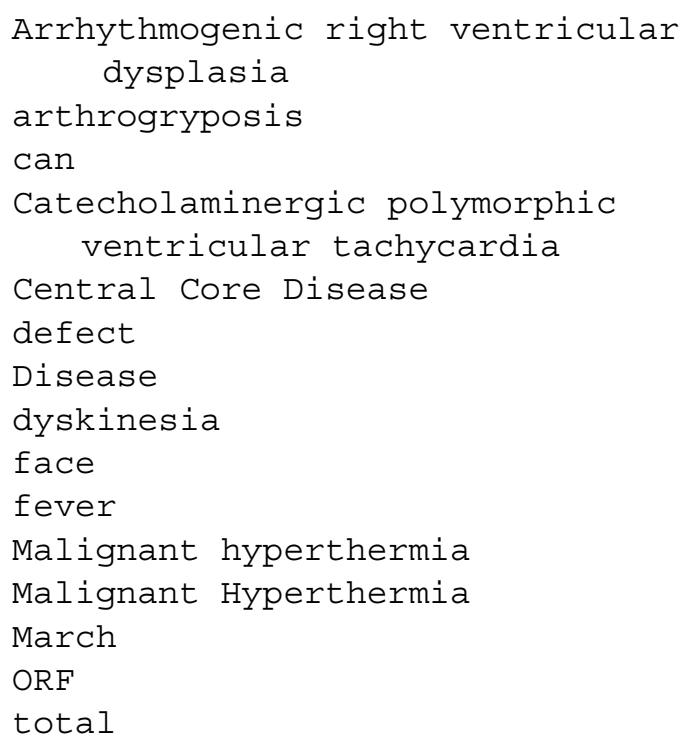

\section{Correct Matches}

Some important diseases could only be recognized by performing a case insensitive match, such as arthrogryposis. This disease was missing because in the lexicon we had the uppercase case version of the labels, but not the lowercase version. We can check it by using the grep command:

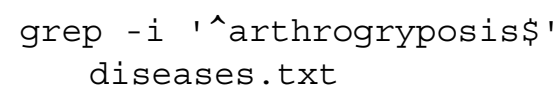

The output does not include the lowercase case version:

\section{Arthrogryposis}

ARTHROGRYPOSIS

We can also check in the text which versions are used:

\$ grep -w -i 'arthrogryposis' chebi_27732_sentences.txt

We can see that only the lowercase version is used:

... (multiple arthrogryposis, congenital dislocation of the hips ...

... fetal akinesia, arthrogryposis multiplex ...

Another example is dyskinesia:

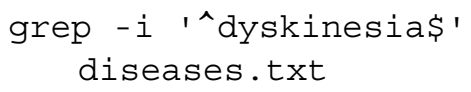

The lexicon has only the disease name with the first character in uppercase:

Dyskinesia

\section{Incorrect Matches}

However, using a case insensitive match may also create other problems, such as the acronym $C A N$ for the disease Crouzon syndrome-acanthosis nigricans syndrome:

\section{grep - $i$ '^CAN\$' diseases.txt}

By using a case insensitive grep we will recognize the common word $C A N$ as a disease. For example, we can check how many times $C A N$ is recognized:

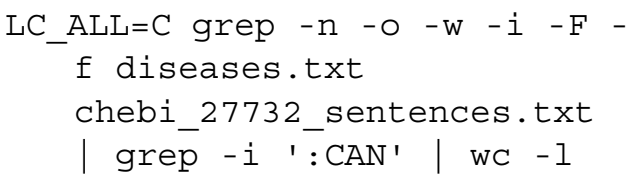

It is recognized 18 times.

And to see which type of matches they are, we can execute the following command:

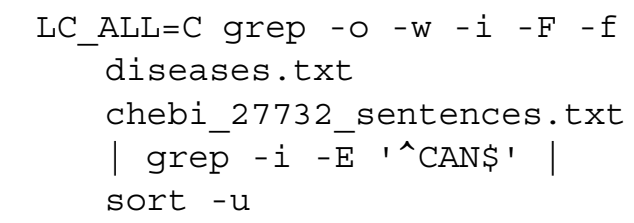

We can verify that the matches are incorrect mentions of the disease acronym:

can

This means we created at least 18 mismatches by performing a case insensitive match.

\section{Entity Linking}

When we are using a generic lexicon, we may be interested in identifying what the recognized labels represent. For example, we may not be aware of what the matched label $A D 2$ represents. 
To solve this issue, we can use our script geturi.sh to perform linking (aka entity disambiguation, entity mapping, normalization), i.e. find the classes in the disease ontology that may be represented by the recognized label. For example, to find what $A D 2$ represents, we can execute the following command:

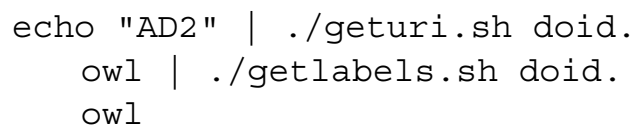

In this case, the result clearly shows that $A D 2$ represents the Alzheimer disease:

AD2

Alzheimer disease 2, late onset

Alzheimer disease associated with APOE4

Alzheimer disease-2

Alzheimer's disease 2

\section{Modified Labels}

However, we may not be so lucky with the labels that were modified by our previous fixes in the lexicon. For example, we can test the case of ATR:

\$ echo "ATR" | ./geturi.sh doid. owl

As expected, we received the warning that no URI was found:

XPath set is empty

An approach to address this issue may involve keeping a track of the original label in a lexicon using another file.

\section{Ambiguity}

We may also have to deal with ambiguity problems where a label may represent multiple terms. For example, if we check how many classes the acronym ATS may represent:

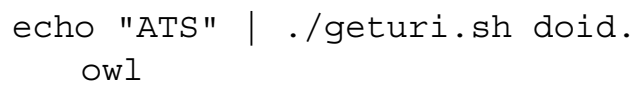

We can see that it may represent two classes: http://purl.obolibrary.org/obo/ DOID_0050434

http://purl.obolibrary.org/obo/

DOID_0110034

These two classes represent two distinct diseases, namely Andersen-Tawil syndrome and X-linked Alport syndrome, respectively.

We can also obtain their alternative labels by providing the two URI as standard input to the getlabels.sh script:

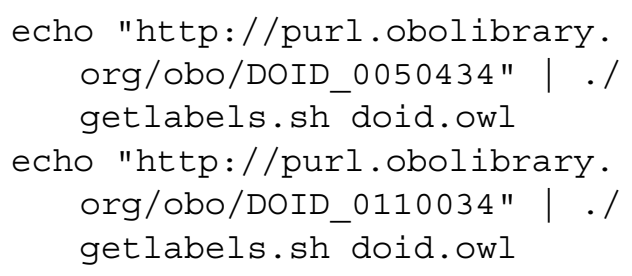

We will get the following two lists, both containing ATS as expected:

\section{ANDERSEN CARDIODYSRHYTHMIC PERIODIC PARALYSIS}

ATS

Andersen syndrome

LQT7

Long QT syndrome 7

Potassium-Sensitive

Cardiodysrhythmic Type

Andersen-Tawil syndrome

\section{ATS}

nephropathy and deafness, $\mathrm{X}$ -

\section{linked}

$\mathrm{X}$-linked Alport syndrome

If we find a ATS mention in the text, the challenge is to identify which of the syndromes the mention refers to. For addressing this challenge, we may have to use advanced entity linking techniques that analyze the context of the text.

\section{Surrounding Entities}

An intuitive solution is to select the class closer in terms of meaning to the others classes mentioned in the surrounding text. This assumes that entities present in a piece of text are somehow semantically related to each other, which is normally 
the case. At least the author assumed some type of relation between them, otherwise the entities would not be in the same sentence.

Let us consider the following sentence about genes and related syndromes from our text file chebi_27732_sentences.txt (on line 436):

... channel genes, KCNQ1 (LQT1), KCNH2 (LQT2), SCN5A (LQT3), KCNE1 (LQT5), and KCNE2 (LQT6 ), along with KCNJ2 (Andersen -Tawil syndrome) and ...

Now assume that the label Andersen-Tawil syndrome been replaced by the acronym ATS:

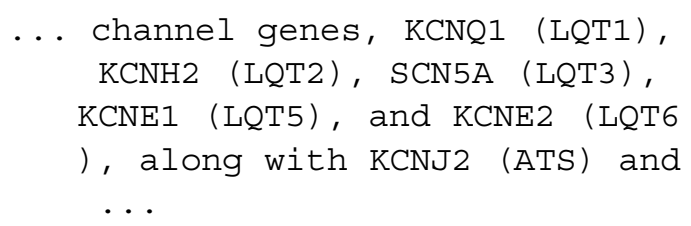

Then, to identify the diseases in the previous sentence, we can execute the following command:

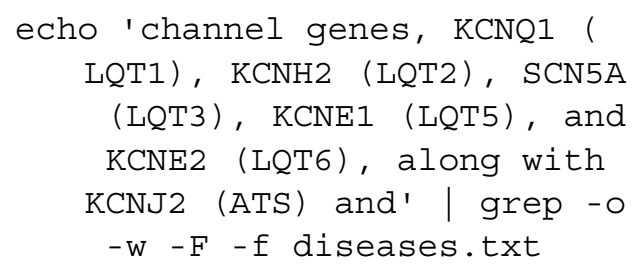

We have a list of labels that can help us decide which is the right class representing ATS:

LQT1

$\mathrm{LQT2}$

LQT3

LQT5

LQT6

ATS

To find their URIs we can use the geturi.sh script:

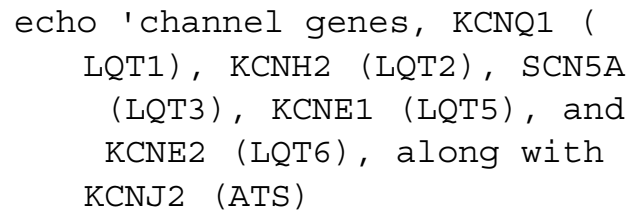

The only ambiguity is for ATS that returns two URIs, one representing the AndersenTawil syndrome (DOID:0050434) and the other representing the X-linked Alport syndrome (DOID:0110034):

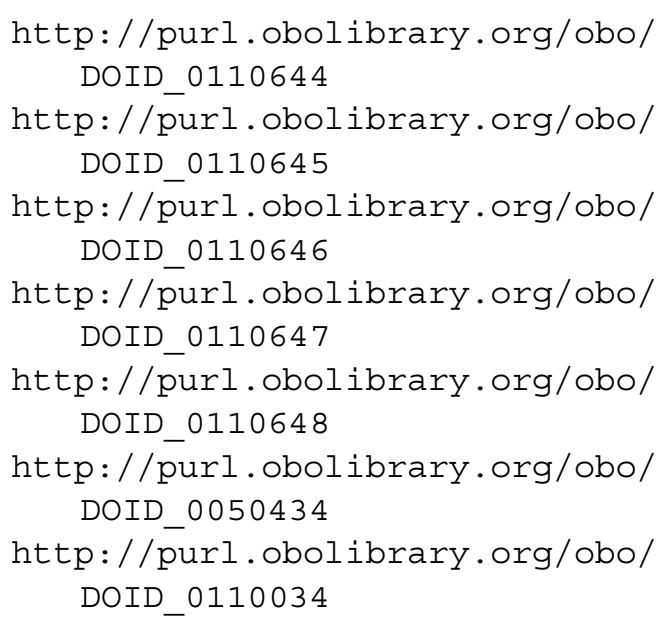

To decide which of the two URIs we should select, we can measure how close in meaning they are to the other diseases also found in the text.

\section{Semantic Similarity}

Semantic similarity measures have been successfully applied to solve these ambiguity problems (Grego and Couto 2013). Semantic similarity quantifies how close two classes are in terms of semantics encoded in a given ontology (Couto and Lamurias 2019). Using the web tool Semantic Similarity Measures using Disjunctive Shared Information (DiShIn) ${ }^{13}$, we can calculate the semantic similarity between our recognized classes. For example, we can calculate the similarity between LQT1 (DOID:0110644) and Andersen-Tawil syndrome (DOID:0050434) (see Fig. 5.6), and the similarity between $L Q T 1$ and X-linked Alport syndrome (DOID:0110034) (see Fig. 5.7).

\section{Measures}

DiShIn provides the similarity values for three measures, namely Resnik, Lin and Jiang-Conrath

\footnotetext{
${ }^{13}$ http://labs.rd.ciencias.ulisboa.pt/dishin/
} 


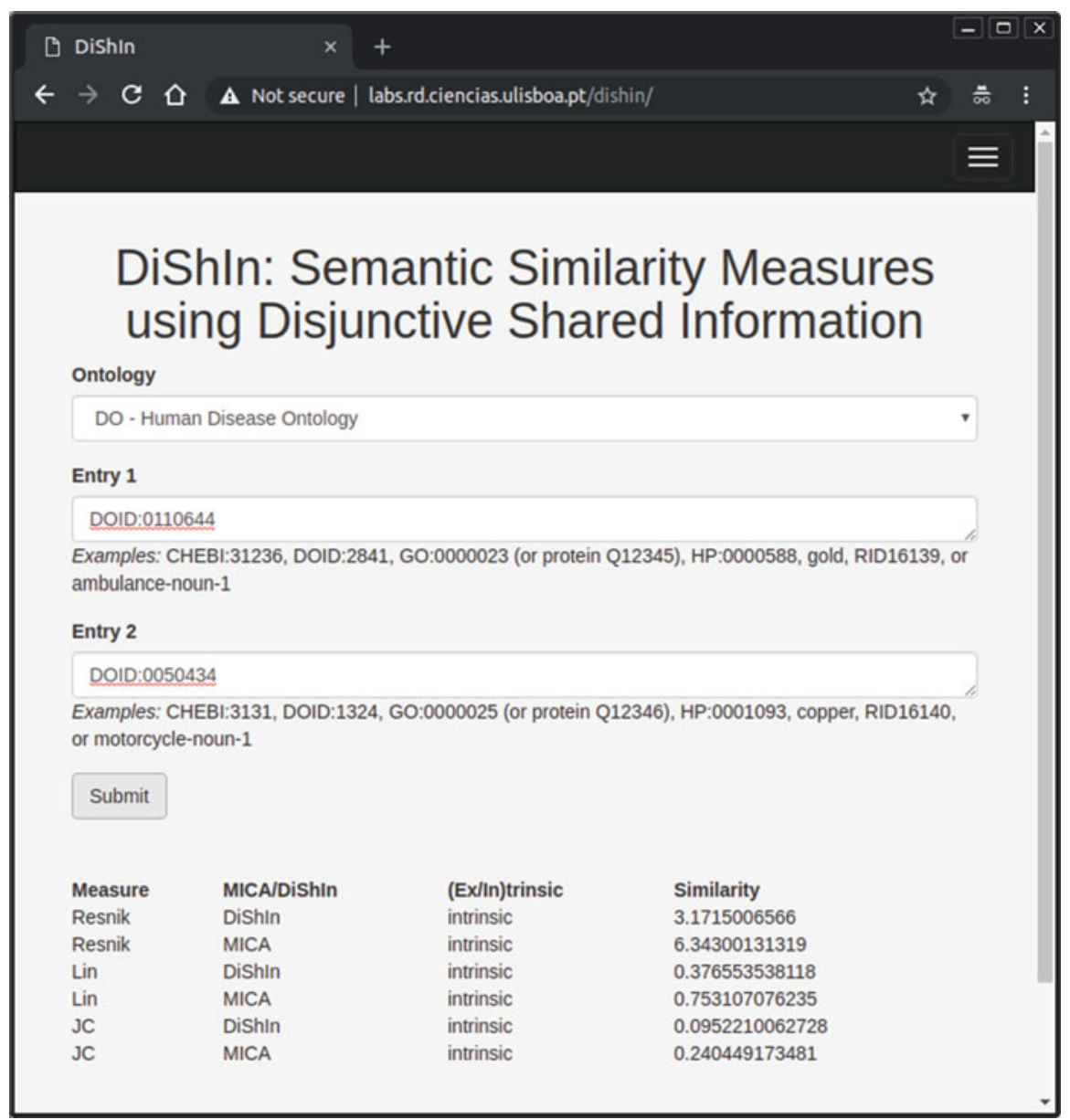

Fig. 5.6 Semantic similarity between LQT1 (DOID:0110644) and Andersen-Tawil syndrome (DOID:0050434) using the online tool DiShIn

(Resnik 1995; Lin et al. 1998; Jiang and Conrath 1997). The last two measures provide values between 0 and 1, and Jiang-Conrath is a distance measure that is converted to similarity.

We can see that for all measures $L Q T 1$ is much more similar to Andersen-Tawil syndrome than to X-linked Alport syndrome. Moreover, JiangConrath's measure gives the only similarity value larger than zero for X-linked Alport syndrome, since it is a converted distance measure. We obtain similar results if we replace $L Q T 1$ by $L Q T 2$, LQT3, LQT5, or LQT6. This means that by using semantic similarity we can identify AndersenTawil syndrome as the correct linked entity for the mention ATS in this text.

\section{DiShIn Installation}

To automatize this process we can also execute DiShIn as a command line ${ }^{14}$, however we may need to install python (or python3) and SQLite ${ }^{15}$.

First, we need to install it locally using the git command line:

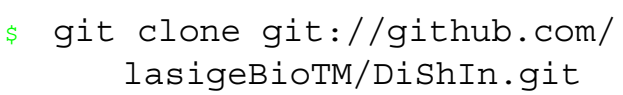

The git command automatically retrieves a tool from the GitHub ${ }^{16}$ software repository.

\footnotetext{
${ }^{14}$ https://github.com/lasigeBioTM/DiShIn

15 apt install python sqlite3 or apt

install python3 sqlite3

${ }^{16}$ https://en.wikipedia.org/wiki/GitHub
} 


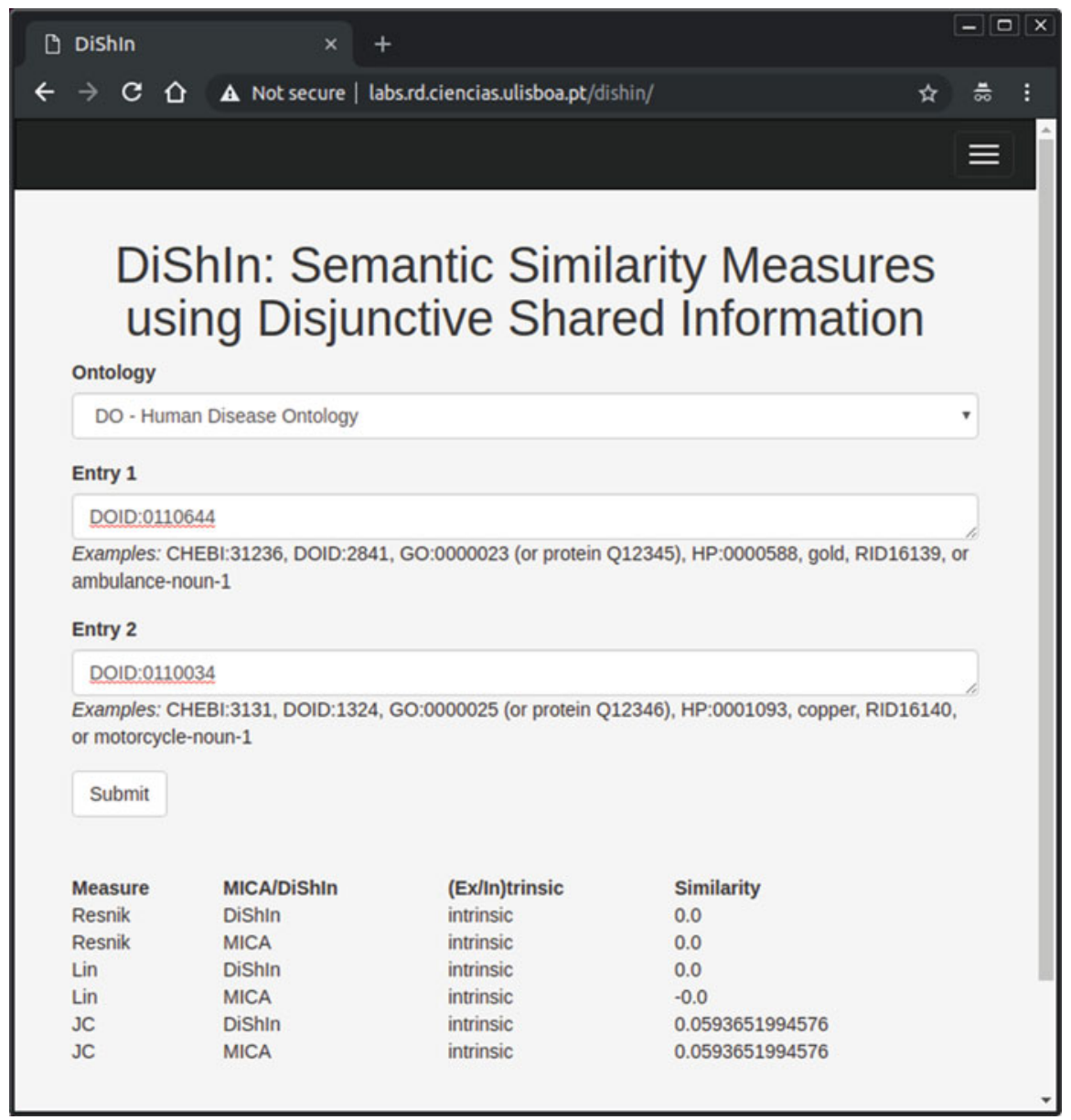

Fig. 5.7 Semantic similarity between LQT1 (DOID:0110644) and X-linked Alport syndrome (DOID:0110034) using the online tool DiShIn

If everything works fine, we should be able to see something like this in our display:

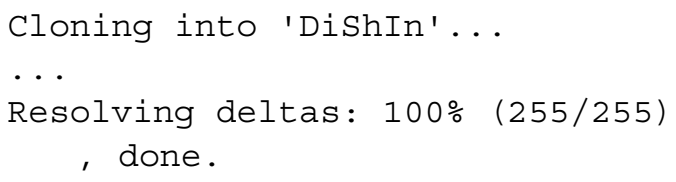

If the git command is not available, we can alternatively download the compressed file (zip), extract its contents and then move to the DiShIn folder:

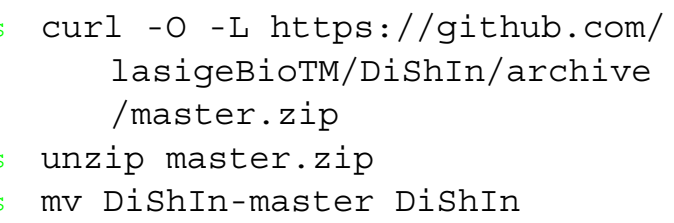

The option $-\mathrm{L}$ enables the curl command to follow a URL redirection ${ }^{17}$. The equivalent long form to the -L option is - location.

We now have to copy the Human Disease Ontology in to the folder using the cp command, and then enter into the DiShIn folder:

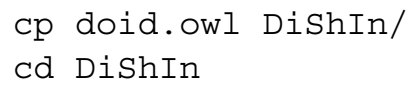

\section{Database File}

To execute DiShIn, we need first to convert the ontology file named doid.owl into a database (SQLite) file named doid.db:

\footnotetext{
${ }^{17}$ https://en.wikipedia.org/wiki/URL_redirection
} 
python dishin.py doid.owl doid .db http://purl.obolibrary .org/obo/ http://www.w3 . org/2000/01/rdf-schema\# subClassof ' '

If the module rdflib is not installed, the following error will be displayed:

ImportError: No module named rdflib

We can try to install it ${ }^{18}$, but this will still take a few minutes to run.

Alternatively, we can download the latest database version:

curl - O http://labs.rd. ciencias.ulisboa.pt/book/ doid.db

\section{DiShIn Execution}

After being installed, we can execute DiShIn by providing the database and two classes identifiers:

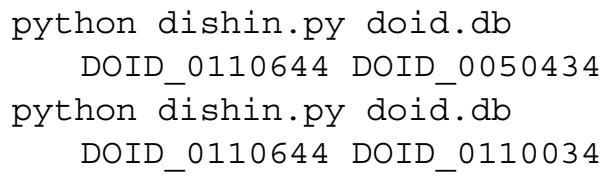

The output of the first command will be the semantic similarity values between $L Q T 1$ (DOID:0110644) and Andersen-Tawil syndrome (DOID:0050434):

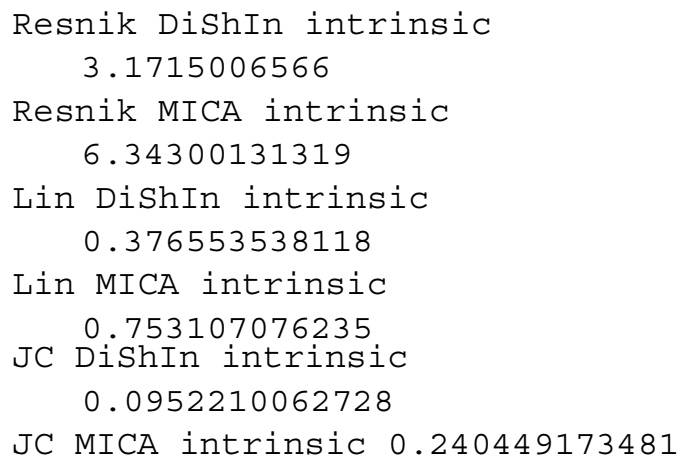

${ }^{18}$ https://github.com/RDFLib/rdflib
The output of the second command will be the semantic similarity values between LQT1 (DOID:0110644) and X-linked Alport syndrome (DOID:0110034):

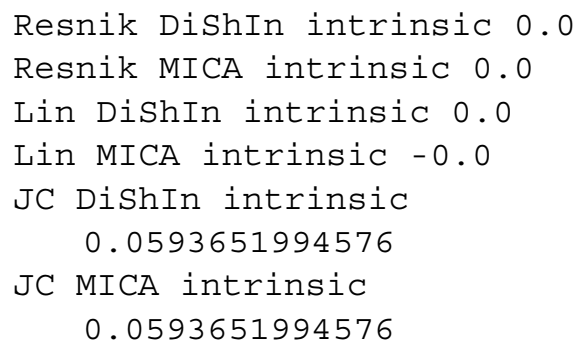

In the end, we should not forget to return to our parent folder:

cd . .

Learning python ${ }^{19}$ and $\mathrm{SQL}^{20}$ is out of scope of this book, but if we do not intend to make any modifications the above steps should be quite simple to execute.

\section{Large Lexicons}

The online tool MER is based on a shell script ${ }^{21}$, so it can be easily executed as a command line to efficiently recognize and link entities using large lexicons.

\section{MER Installation}

First, we need to install it locally using the git command line:

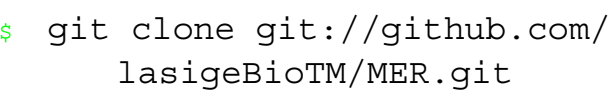

If everything works fine, we should be able to see something like this in our display:

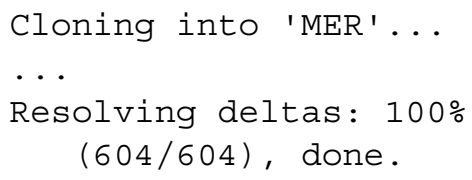

\footnotetext{
${ }^{19} \mathrm{https}: / /$ www.w3schools.com/python/

${ }^{20} \mathrm{https} / / / \mathrm{www} . w 3$ schools.com/sql/

${ }^{21}$ https://github.com/lasigeBioTM/MER
} 
If the git command is not available, we can alternatively download the compressed file (zip), and extract its contents:

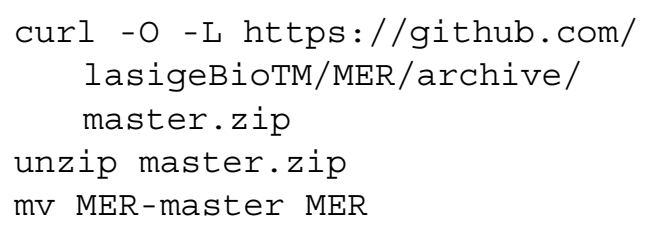

We now have to copy the Human Disease Ontology in to the data folder of MER, and then enter into the MER folder:

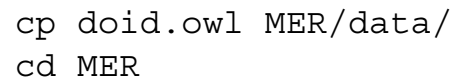

\section{Lexicon Files}

To execute MER, we need first to create the lexicon files:

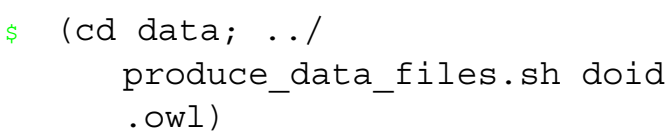

This may take a few minutes to run. However, we only need to execute it once, each time we want to use a new version of the ontology. If we wait, the output will include the last patterns of each of the lexicon files.

Alternatively, we can download the lexicon files, and extract them into the data folder:

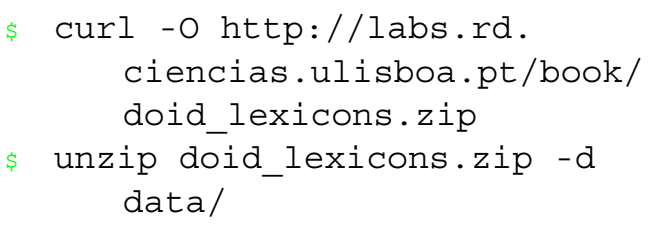

We can check the contents of the created lexicons by using the tail command:

\section{tail data/doid*}

These patterns are created according to the number of words of each term.

The output should be something like this:

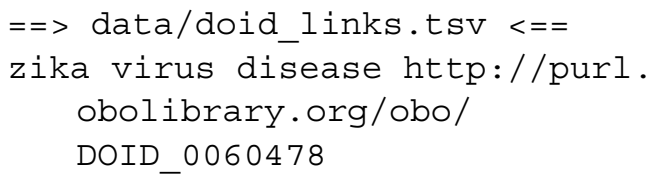

zikv congenital infection http ://purl.obolibrary.org/obo/ DOID_0080180

zinacef allergy http://purl. obolibrary.org/obo/ DOID_0040025

zinsser-cole-engman syndrome http: //purl.obolibrary.org/ obo/DOID_0070025

ziziphus mauritiana fruit allergy http://purl. obolibrary.org/obo/ DOID_0060507

zlotogora-zilberman-tenenbaum syndrome http://purl. obolibrary.org/obo/ DOID_0060773

zollinger-ellison syndrome http ://purl.obolibrary.org/obo/ DOID_0050782

zoophilia http://purl.obolibrary .org/obo/DOID_9336

zoophobia http://purl.obolibrary .org/obo/DOID_600

zygomycosis http://purl. obolibrary.org/obo/DOID_8485

$==>$ data/doid.txt $<==$

zika virus disease

zikv congenital infection

zinacef allergy

zinsser-cole-engman syndrome ziziphus mauritiana fruit allergy

zlotogora-zilberman-tenenbaum syndrome

zollinger-ellison syndrome zoophilia zoophobia zygomycosis

$==>$ data/doid_wordl.txt $<==$ xph

xpid

xpv

xscid

yaba 
yaws

zaspopathy

zoophilia

zoophobia

zygomycosis

$==>$ data/doid_word2.txt $<==$ yunis.varon syndrome

zantac allergy

zebrafish allergy

zellweger syndrome

zemuron allergy

zika fever

zinacef allergy

zinsser.cole.engman syndrome

zlotogora.zilberman. tenenbaum

syndrome

zollinger.ellison syndrome

$==>$ data/doid_words2.txt $<==$

yersinia infectious

yersinia pestis

yersinia pseudotuberculosis

y.linked monogenic

y.linked sertoli

y.linked spermatogenic

yolk sac

zika virus

zikv congenital

ziziphus mauritiana

$==>$ data/doid_words.txt $<==$

y.linked spermatogenic failure 1

y.linked spermatogenic failure 2

yolk sac neoplasm

yolk sac tumor

yolk sac tumor of mediastinum

yolk sac tumor of the cns

zika virus congenital syndrome

zika virus disease

zikv congenital infection

ziziphus mauritiana fruit allergy

\section{MER Execution}

Now we are ready to execute MER, by providing each sentence from the file chebi_27732_sentences.txt as argument to its get_entities.sh script.

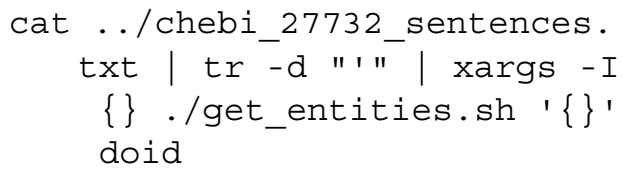

We removed single quotes from the text, since they are special characters to the command line xargs. We should note that this is the get_entities.sh script inside the MER folder, not the one we created before.

Now we will be able to obtain a large number of matches:

89111 malignant hyperthermia http: //purl.obolibrary.org/ obo/DOID_8545

7496 malignant hyperthermia http: //purl.obolibrary.org/ obo/DOID_8545

157164 disease http://purl. obolibrary.org/obo/DOID_4

144164 central core disease http: //purl.obolibrary.org/ obo/DOID_3529

1320 disease http://purl. obolibrary.org/obo/DOID_4

4755 myopathy http://purl. obolibrary.org/obo/DOID_423

The first two numbers represent the start and end position of the match in the sentence. They are followed by the name of the disease and its URI in the ontology.

We can also redirect the output to a TSV file named diseases_recognized.tsv:

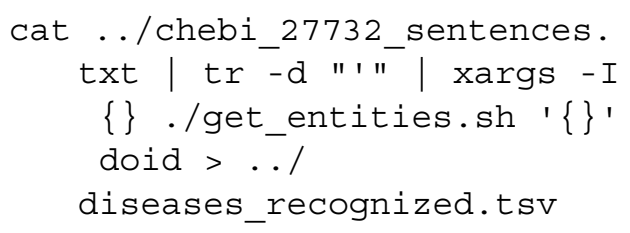




\begin{tabular}{|c|c|c|c|}
\hline & A & B & D \\
\hline 1 & 89 & 111 malignant hyperthermia & http://purl.obolibrary.org/obo/DOID_8545 \\
\hline 2 & 74 & 96 malignant hyperthermia & http://purl.obolibrary.org/obo/DOID_8545 \\
\hline 3 & 157 & 164 disease & http://purl.obolibrary.org/obo/DOID_4 \\
\hline 4 & 144 & 164 central core disease & http://purl.obolibrary.org/obo/DOID_3529 \\
\hline 5 & 13 & 20 disease & http://purl.obolibrary.org/obo/DOID_4 \\
\hline 6 & 47 & 55 myopathy & http://purl.obolibrary.org/obo/DOID 423 \\
\hline
\end{tabular}

Fig. 5.8 The diseases_recognized.tsv file opened in a spreadsheet application

We can now open the file in our spreadsheet application, such as LibreOffice Calc or Microsoft Excel (see Fig. 5.8).

Again, we should not forget to return to our parent folder in the end:

$\$ \mathrm{~cd} .$.

\section{Further Reading}

To know more about biomedical ontologies, the book entitled Introduction to bio-ontologies is an excellent option, covering most of the ontologies and computational techniques exploring them (Robinson and Bauer 2011).

Another approach is to read and watch the materials of the training course given by Barry Smith ${ }^{22}$.

\footnotetext{
${ }^{22}$ http://ontology.buffalo.edu/smith/ IntroOntology_Course.html
}

Open Access This chapter is licensed under the terms of the Creative Commons Attribution 4.0 International License (http://creativecommons.org/licenses/by/4.0/), which permits use, sharing, adaptation, distribution and reproduction in any medium or format, as long as you give appropriate credit to the original author(s) and the source, provide a link to the Creative Commons licence and indicate if changes were made.

The images or other third party material in this chapter are included in the chapter's Creative Commons licence, unless indicated otherwise in a credit line to the material. If material is not included in the chapter's Creative Commons licence and your intended use is not permitted by statutory regulation or exceeds the permitted use, you will need to obtain permission directly from the copyright holder. 


\section{Bibliography}

Allen G, Owens M (2011) The definitive guide to SQLite. Books for professionals by professionals. Apress, Berkeley

Angermueller C, Pärnamaa T, Parts L, Stegle O (2016) Deep learning for computational biology. Mol Syst Biol 12(7):878

Aramaki E, Maskawa S, Morita M (2011) Twitter catches the flu: detecting influenza epidemics using twitter. In: Proceedings of the conference on empirical methods in natural language processing. Association for Computational Linguistics, pp 1568-1576

Aras H, Hackl-Sommer R, Schwantner M, Sofean M (2014) Applications and challenges of text mining with patents. In: IPaMin@ KONVENS

Ashburner M, Ball CA, Blake JA, Botstein D, Butler H, Cherry JM, Davis AP, Dolinski K, Dwight SS, Eppig JT et al (2000) Gene ontology: tool for the unification of biology. Nat Genet 25(1):25

Baker J, Milligan I (2014) Counting and mining research data with unix. Technical report, The Editorial Board of the Programming Historian

Barros M, Couto FM (2016) Knowledge representation and management: a linked data perspective. Yearb Med Inform 25(1):178-183

Blumenthal D, Tavenner M (2010) The meaningful useİregulation for electronic health records. $\mathrm{N}$ Engl J Med 363(6):501-504

Borst W, Borst W (1997) Construction of engineering ontologies for knowledge sharing and reuse. Ph.D. thesis, University of Twente

Campos L, Pedro V, Couto F (2017) Impact of translation on named-entity recognition in radiology texts. Database 2017:bax064

Canese K (2006) Pubmed celebrates its 10th anniversary. NLM Tech Bull 352:e5

Ching T, Himmelstein DS, Beaulieu-Jones BK, Kalinin AA, Do BT, Way GP, Ferrero E, Agapow P-M, Zietz M, Hoffman MM et al (2018) Opportunities and obstacles for deep learning in biology and medicine. J R Soc Interface 15(141):20170387

Cock PJ, Antao T, Chang JT, Chapman BA, Cox CJ, Dalke A, Friedberg I, Hamelryck T, Kauff F, Wilczynski B et al (2009) Biopython: freely available python tools for computational molecular biology and bioinformatics. Bioinformatics 25(11):1422-1423

Cook CE, Bergman MT, Cochrane G, Apweiler R, Birney $E$ (2017) The european bioinformatics institute in 2017: data coordination and integration. Nucleic Acids Res 46(D1):D21-D29

Coordinators NR (2018) Database resources of the national center for biotechnology information. Nucleic Acids Res 46(Database issue):D8

Couto F, Lamurias A (2018) MER: a shell script and annotation server for minimal named entity recognition and linking. J Cheminfo 10(1):58

Couto F, Lamurias A (2019) Semantic similarity definition. In: Ranganathan S, Nakai K, Schönbach C, Gribskov M (eds) Encyclopedia of bioinformatics and computational biology, vol 1. Oxford: Elsevier

Couto FM, Campos LF, Lamurias A (2017) Mer: a minimal named-entity recognition tagger and annotation server. Proc BioCreative 5:130-7

Couto FM, Silva MJ, Lee V, Dimmer E, Camon E, Apweiler R, Kirsch H, Rebholz-Schuhmann D (2006) GOAnnotator: linking protein go annotations to evidence text. J Biomed Discov Collab 1(1):19

Degtyarenko K, De Matos P, Ennis M, Hastings J, Zbinden M, McNaught A, Alcántara R, Darsow M, Guedj M, Ashburner M (2007) ChEBI: a database and ontology for chemical entities of biological interest. Nucleic Acids Res 36(suppl_1):D344-D350

Doms A, Schroeder M (2005) GoPubMed: exploring pubmed with the gene ontology. Nucleic Acids Res 33(suppl_2):W783-W786

Ferreira JD, Inácio B, Salek RM, Couto FM (2017) Assessing public metabolomics metadata, towards improving quality. J Integr Bioinform 14(4):67-72

Forta B (2018) Learning regular expressions. AddisonWesley Professional, Boston

Gentleman RC, Carey VJ, Bates DM, Bolstad B, Dettling M, Dudoit S, Ellis B, Gautier L, Ge Y, Gentry J et al (2004) Bioconductor: open software development for computational biology and bioinformatics. Genome Biol 5(10):R80 
Grego T, Couto FM (2013) Enhancement of chemical entity identification in text using semantic similarity validation. PloS one 8(5):e62984

Gruber TR (1993) A translation approach to portable ontology specifications. Knowl Acquis 5(2):199-220

Haines N (2017) Beginning Ubuntu for Windows and Mac users: start your journey into free and open source software. Apress, Berkeley

Hersh W (2008) Information retrieval: a health and biomedical perspective. Springer Science \& Business Media, New York

Hey T, Tansley S, Tolle KM et al (2009) The fourth paradigm: data-intensive scientific discovery, vol 1. Microsoft research Redmond, Redmond

Holzinger A, Jurisica I (2014) Knowledge discovery and data mining in biomedical informatics: the future is in integrative, interactive machine learning solutions. In: Interactive knowledge discovery and data mining in biomedical informatics. Springer, Heidelberg, pp 1-18

Holzinger A, Schantl J, Schroettner M, Seifert C, Verspoor K (2014) Biomedical text mining: state-of-theart, open problems and future challenges. In: Holzinger A, Jurisica I (eds) Interactive knowledge discovery and data mining in biomedical informatics. Springer, Heidelberg, pp 271-300

Hunter L, Cohen KB (2006) Biomedical language processing: what's beyond pubmed? Mol Cell 21(5):589594

Jensen PB, Jensen LJ, Brunak S (2012) Mining electronic health records: towards better research applications and clinical care. Nat Rev Genet 13(6):395

Jiang JJ, Conrath DW (1997) Semantic similarity based on corpus statistics and lexical taxonomy. In: Proceedings of the 10th research on computational linguistics international conference, pp 19-33

Jurafsky D, Martin JH (2014) Speech and language processing, vol 3. Pearson, London

Kleene SC (1951) Representation of events in nerve nets and finite automata. Technical report, Rand Project Air Force, Santa Monica

Krallinger M, Rabal O, Lourenço A, Oyarzabal J, Valencia A (2017) Information retrieval and text mining technologies for chemistry. Chem Rev 117(12):7673-7761

Lamurias A, Couto F (2019) Text mining for bioinformatics using biomedical literature. In: Ranganathan S, Nakai K, Schönbach C, Gribskov M (eds) Encyclopedia of bioinformatics and computational biology, vol 1. Elsevier, Oxford

Lamurias A, Ferreira JD, Clarke LA, Couto FM (2017) Generating a tolerogenic cell therapy knowledge graph from literature. Front Immunol 8:1656

Leonelli S (2016) Data-centric biology: a philosophical study. University of Chicago Press, Chicago

Lesk A (2014) Introduction to bioinformatics. Oxford University Press, Oxford

Li W, Cowley A, Uludag M, Gur T, McWilliam H, Squizzato S, Park YM, Buso N, Lopez R (2015) The embl-ebi bioinformatics web and programmatic tools framework. Nucleic Acids Res 43(W1):W580-W584
Lin D et al (1998) An information-theoretic definition of similarity. In: Icml, vol 98, pp 296-304. Citeseer

Lu Z (2011) PubMed and beyond: a survey of web tools for searching biomedical literature. Database 2011:baq036

McGuinness DL, Van Harmelen F et al (2004) OWL web ontology language overview. W3C Recommendation 10(10):2004

Nosek BA, Alter G, Banks GC, Borsboom D, Bowman SD, Breckler SJ, Buck S, Chambers CD, Chin G, Christensen G et al (2015) Promoting an open research culture. Science 348(6242):1422-1425

Ong E, Xiang Z, Zhao B, Liu Y, Lin Y, Zheng J, Mungall C, Courtot M, Ruttenberg A, He Y (2016) Ontobee: a linked ontology data server to support ontology term dereferencing, linkage, query and integration. Nucleic Acids Res 45(D1):D347-D352

Rawat S, Meena S (2014) Publish or perish: where are we heading? J Res Med Sci 19(2):87

Rebholz-Schuhmann D, Kirsch H, Couto F (2005) Facts from text-is text mining ready to deliver? PLoS Biol 3(2):e65

Resnik P (1995) Using information content to evaluate semantic similarity in a taxonomy. In: Proceedings of the 14th international joint conference on artificial intelligence, vol 1, pp 448-453. Morgan Kaufmann Publishers Inc.

Richardson L, Ruby S (2008) RESTful web services. O’Reilly Media, Inc., Sebastopol

Ritchie DM (1971) Unix programmer's manual. Technical report, Technical report Bell

Robinson PN, Bauer S (2011) Introduction to bioontologies. Chapman and Hall/CRC, Boca Raton

Schriml LM, Mitraka E, Munro J, Tauber B, Schor M, Nickle L, Felix V, Jeng L, Bearer C, Lichenstein R et al (2018) Human disease ontology 2018 update: classification, content and workflow expansion. Nucleic Acids Res 47:D955-D962

Schuemie MJ, Weeber M, Schijvenaars BJ, van Mulligen EM, van der Eijk CC, Jelier R, Mons B, Kors JA (2004) Distribution of information in biomedical abstracts and full-text publications. Bioinformatics 20(16):2597-2604

Shah, P. K., Perez-Iratxeta, C., Bork, P., \& Andrade, M. A. (2003). Information extraction from full text scientific articles: where are the keywords? BMC Bioinformatics 4(1):20

Shotts WE Jr (2012) The Linux command line: a complete introduction. No Starch Press, San Francisco

Singhal A (2012) Introducing the knowledge graph: things, not strings. Official Google Blog 5. https:// googleblog.blogspot.com/2012/05/introducingknowledge-graph-things-not.html

Smith B, Ashburner M, Rosse C, Bard J, Bug W, Ceusters W, Goldberg LJ, Eilbeck K, Ireland A, Mungall CJ et al (2007) The obo foundry: coordinated evolution of ontologies to support biomedical data integration. Nat Biotechnol 25(11):1251 
Spasic I, Ananiadou S, McNaught J, Kumar A (2005) Text mining and ontologies in biomedicine: making sense of raw text. Brief Bioinform 6(3):239-251

Stajich JE, Block D, Boulez K, Brenner SE, Chervitz SA, Dagdigian C, Fuellen G, Gilbert JG, Korf I, Lapp H et al (2002) The bioperl toolkit: Perl modules for the life sciences. Genome Res 12(10):1611-1618

Stephens ZD, Lee SY, Faghri F, Campbell RH, Zhai C, Efron MJ, Iyer R, Schatz MC, Sinha S, Robinson GE (2015) Big data: astronomical or genomical? PLoS Biol 13(7):e1002195

Studer R, Benjamins VR, Fensel D et al (1998) Knowledge engineering: principles and methods. Data Knowl Eng 25(1):161-198

Styler IV WF, Bethard S, Finan S, Palmer M, Pradhan S, de Groen PC, Erickson B, Miller T, Lin C, Savova G et al (2014) Temporal annotation in the clinical domain. Trans Assoc Comput Ling 2:143

Tomczak A, Mortensen JM, Winnenburg R, Liu C, Alessi DT, Swamy V, Vallania F, Lofgren S, Haynes W, Shah $\mathrm{NH}$ et al (2018) Interpretation of biological experiments changes with evolution of the gene ontology and its annotations. Sci Rep 8(1):5115

Wei C-H, Kao H-Y, Lu Z (2013) PubTator: a web-based text mining tool for assisting biocuration. Nucleic Acids Res 41(W1):W518-W522

Wu D, Fung P (1994) Improving chinese tokenization with linguistic filters on statistical lexical acquisition. In: Proceedings of the 4th conference on applied natural language processing

Yeh A, Hirschman L, Morgan A (2003) Evaluation of text data mining for database curation: lessons learned from the KDD challenge cup. Bioinformatics 19(1):i331i339 


\section{Index}

A

Ancestors, 8, 74-78

B

Bibliographic databases, 2, 10

Bioinformatics, 1, 7, 10, 15

Biomedical data repositories, 1,10

Biomedical literature, 10, 60

\section{C}

Chemical entities of biological interest (ChEBI), 13-15, $17,19,20,22,29-32,37,38,41,42,47,57,61$, $66,67,74,76,82$

Client uniform resource locator (cURL), 7, 30-36, 41, $42,61,87-89$

Command line tools, 6-8, 11, 15, 24-28, 30-32, 35

Comma-separated values (CSV), 6, 7, 15, 20, 29-35

Controlled vocabularies, 12-14

D

Data

extraction, 32

filtering, 33

selection, 32,34

Directed acyclic graphs (DAG), 12, 13, 72

Disease Ontology (DO), 13, 22, 61, 63, 66, 74, 78, 84, 87,89

\section{E}

Entity, 2, 8, 17, 57-59, 62, 74, 76, 83-87 linking, 8, 83-84

European bioinformatics institute (EBI), 1, 7, 10, 17

Evaluation metrics, 47

Extensible markup language (XML), 6, 7, 14, 15, 20, 21, $29,34,36,37,39-42,62$

\section{$\mathbf{L}$}

Lexicons, 8, 22, 59, 76-84, 88, 89

$\mathbf{N}$

Named-entity recognition (NER), 8

Natural language processing (NLP), 55

O

Ontologies, 2, 7, 8, 10, 12-15, 17, 22, 23, 61-63, 65, 66, $72,76,78,84,85,87,89,90$

Open biomedical ontologies (OBO), 12-14

OWL, see Web ontology language (OWL)

$\mathbf{P}$

Pattern matching, 8, 34, 45, 48, 49, 56, 82

Programmatic access, 11, 30

$\mathbf{R}$

Recursion, 74-75

Regular expressions, 8, 32, 48-51, 53, 55, 57, 78, 80

Relation extraction, 8,59

$\mathbf{S}$

Semantics, 2, 4, 7-13, 61-91

Semantic resources, 10, 61

Semantic similarity, 12, 85-88

Shell scripting, 5-8, 17, 24, 43, 45,88

Spreadsheet applications, 6, 7, 25, 32, 91

String matching, 50, 53, 67, 78

T

Tab-separated values (TSV), 6, 7, 20, 58, 90

Terminal application, 24-26 
Text files, 6, 7, 26, 32, 48, 85

Text mining, 4, 10, 22, 59

Tokenization, 8, 55

U

Uniform resource identifier (URI), 15, 65-69, 71-75, 78, $84,85,90$

UniProt citations service, 10, 11, 22, 41

Unix shell, 5, 24-26, 82
W

Web ontology language (OWL), 12, 14-15, 61-62, $65-68,72,78$

Web retrieval, 30

Word matching, 47, 48, 50

$\mathbf{X}$

XML, see Extensible markup language (XML)

XML path language (XPath), 39-41, 62, 64-68, 70-75, $78,81,84$ 